\title{
a-Glucosidase and Protein Tyrosine Phosphatase 1B Inhibitors from Malbranchea circinata
}

Manuel Rangel-Grimaldo, ${ }^{\dagger} \pitchfork$ Martha L. Macías-Rubalcava, ${ }^{\perp}$ Martin González-Andrade, ${ }^{\S}$ Huzefa Raja, ${ }^{¥}$ Mario Figueroa, ${ }^{\dagger}$ and Rachel Mata*, ${ }^{*}$

†Facultad de Química, ${ }^{\perp}$ Instituto de Química, and §Facultad de Medicina, Universidad Nacional Autónoma de México, Ciudad de México 04510, México.

${ }^{¥}$ Department of Chemistry and Biochemistry, University of North Carolina Greensboro, Greensboro, North Carolina 27402, United States

\section{SUPPORTING INFORMATION}

\section{List of Contents}

Figure S1. ${ }^{1} \mathrm{H}$ NMR spectrum of 1 in $\mathrm{CD}_{3} \mathrm{OD}(700 \mathrm{MHz})$.

Figure S2. ${ }^{13} \mathrm{C}$ NMR spectrum of 1 in $\mathrm{CD}_{3} \mathrm{OD}(175 \mathrm{MHz})$.

Figure S3. HSQC spectrum of 1 in $\mathrm{CD}_{3} \mathrm{OD}$.

Figure S4. $\mathrm{HMBC}$ spectrum of 1 in $\mathrm{CD}_{3} \mathrm{OD}$.

Figure S5. COSY spectrum of 1 in $\mathrm{CD}_{3} \mathrm{OD}$.

Figure S6. NOESY spectrum of 1 in $\mathrm{CD}_{3} \mathrm{OD}$.

Figure S7. HRESIMS spectrum of $\mathbf{1}$.

Figure S8. ${ }^{1} \mathrm{H}$ NMR spectrum of 2 in $\mathrm{CD}_{3} \mathrm{OD}(700 \mathrm{MHz})$.

Figure S9. ${ }^{13} \mathrm{C}$ NMR spectrum of 2 in $\mathrm{CD}_{3} \mathrm{OD}(175 \mathrm{MHz})$.

Figure S10. $\mathrm{HSQC}$ spectrum of 2 in $\mathrm{CD}_{3} \mathrm{OD}$.

Figure S11. $\mathrm{HMBC}$ spectrum of $\mathbf{2}$ in $\mathrm{CD}_{3} \mathrm{OD}$.

Figure S12. COSY spectrum of 2 in $\mathrm{CD}_{3} \mathrm{OD}$.

Figure S13. NOESY spectrum of $\mathbf{2}$ in $\mathrm{CD}_{3} \mathrm{OD}$.

Figure S14. HRESIMS spectrum of 2.

Figure S15. ${ }^{1} \mathrm{H}$ NMR spectrum of 3 in $\mathrm{CDCl}_{3}(700 \mathrm{MHz})$.

Figure S16. ${ }^{13} \mathrm{C}$ NMR spectrum of 3 in $\mathrm{CDCl}_{3}+$ drops of $\mathrm{CD}_{3} \mathrm{OD}(175 \mathrm{MHz})$.

Figure S17. HSQC spectrum of 3 in $\mathrm{CDCl}_{3}+$ drops of $\mathrm{CD}_{3} \mathrm{OD}$.

Figure S18. HMBC spectrum of $\mathbf{3}$ in $\mathrm{CDCl}_{3}+$ drops of $\mathrm{CD}_{3} \mathrm{OD}$.

Figure S19. COSY spectrum of $\mathbf{3}$ in $\mathrm{CDCl}_{3}+$ drops of $\mathrm{CD}_{3} \mathrm{OD}$.

Figure S20. NOESY spectrum of $\mathbf{3}$ in $\mathrm{CDCl}_{3}+$ drops of $\mathrm{CD}_{3} \mathrm{OD}$.

Figure S21. HRESIMS spectrum of 3.

Figure S22. ${ }^{1} \mathrm{H}$ NMR spectrum of 4 in $\mathrm{CDCl}_{3}(400 \mathrm{MHz})$.

Figure S23. ${ }^{13} \mathrm{C}$ NMR spectrum of 4 in $\mathrm{CDCl}_{3}(100 \mathrm{MHz})$.

Figure S24. HSQC spectrum of $\mathbf{4}$ in $\mathrm{CDCl}_{3}$.

Figure S25. HMBC spectrum of 4 in $\mathrm{CDCl}_{3}$.

Figure S26. COSY spectrum of 4 in $\mathrm{CDCl}_{3}$.

Figure S27. NOESY spectrum of 4 in $\mathrm{CDCl}_{3}$.

Figure S28. HRESIMS spectrum of 4.

Figure S29. ${ }^{1} \mathrm{H}$ NMR spectrum of 5 in $\mathrm{CDCl}_{3}(700 \mathrm{MHz})$.

Figure S30. ${ }^{13} \mathrm{C}$ NMR spectrum of 5 in $\mathrm{CDCl}_{3}(175 \mathrm{MHz})$.

Figure S31. HSQC spectrum of 5 in $\mathrm{CDCl}_{3}$.

Figure S32. HMBC spectrum of 5 in $\mathrm{CDCl}_{3}$.

Figure S33. COSY spectrum of 5 in $\mathrm{CDCl}_{3}$.

Figure S34 NOESY spectrum of 5 in $\mathrm{CDCl}_{3}$.

Figure S35. HRESIMS spectrum of 5. 
Figure S36. ${ }^{1} \mathrm{H}$ NMR spectrum of 6 in $\mathrm{CDCl}_{3}(700 \mathrm{MHz})$.

Figure S37. ${ }^{13} \mathrm{C}$ NMR spectrum of 6 in $\mathrm{CDCl}_{3}(175 \mathrm{MHz})$.

Figure S38. HSQC spectrum of $\mathbf{6}$ in $\mathrm{CDCl}_{3}$.

Figure S39. $\mathrm{HMBC}$ spectrum of 6 in $\mathrm{CDCl}_{3}$.

Figure S40. COSY spectrum of 6 in $\mathrm{CDCl}_{3}$.

Figure S41. NOESY spectrum of 6 in $\mathrm{CDCl}_{3}$.

Figure S42. HRESIMS spectrum of 6 .

Figure S43. ${ }^{1} \mathrm{H}$ NMR spectrum of 7 in $\mathrm{CDCl}_{3}(700 \mathrm{MHz})$.

Figure S44. ${ }^{13} \mathrm{C}$ NMR spectrum of 7 in $\mathrm{CDCl}_{3}(175 \mathrm{MHz})$.

Figure S45. HSQC spectrum of 7 in $\mathrm{CDCl}_{3}$.

Figure S46. HMBC spectrum of 7 in $\mathrm{CDCl}_{3}$.

Figure S47. COSY spectrum of 7 in $\mathrm{CDCl}_{3}$.

Figure S48. NOESY spectrum of 7 in $\mathrm{CDCl}_{3}$.

Figure S49. HRESIMS spectrum of 7.

Figure S50. ${ }^{1} \mathrm{H}$ NMR spectrum of $\mathbf{8}$ in $\mathrm{CDCl}_{3}(700 \mathrm{MHz})$.

Figure S51. ${ }^{13} \mathrm{C}$ NMR spectrum of 8 in $\mathrm{CDCl}_{3}(175 \mathrm{MHz})$.

Figure S52. HSQC spectrum of 8 in $\mathrm{CDCl}_{3}$.

Figure S53. $\mathrm{HMBC}$ spectrum of $\mathbf{8}$ in $\mathrm{CDCl}_{3}$.

Figure S54. COSY spectrum of 8 in $\mathrm{CDCl}_{3}$.

Figure S55. NOESY spectrum of 8 in $\mathrm{CDCl}_{3}$.

Figure S56. HRESIMS spectrum of 8 .

Figure S57. ${ }^{1} \mathrm{H}$ NMR spectrum of 9 in $\mathrm{CD}_{3} \mathrm{OD}(700 \mathrm{MHz})$.

Figure S58. ${ }^{1} \mathrm{H}$ NMR spectrum of 10 in $\mathrm{CD}_{3} \mathrm{OD}(700 \mathrm{MHz})$.

Figure S59. ${ }^{1} \mathrm{H}$ NMR spectrum of 11 and 12 in $\mathrm{CDCl}_{3}(700 \mathrm{MHz})$.

Figure S60. Sucrose tolerance test of malbrancheamide (10).

Figure S61. Docking analysis for $1(\alpha \mathrm{GHY})$.

Figure S62. Docking analysis for 3 ( $\alpha \mathrm{GHY})$.

Figure S63. Docking analysis for 9 ( $\alpha \mathrm{GHY})$.

Figure S64. Docking analysis for $10(\alpha \mathrm{GHY})$.

Figure S65. Docking analysis for acarbose ( $\alpha \mathrm{GHY})$.

Figure S66. Docking analysis for 1 in (PTP-1B).

Figure S67. Docking analysis for 2 (PTP-1B).

Figure S68. Docking analysis for 3 (PTP-1B).

Figure S69. Docking analysis for 4 (PTP-1B).

Figure S70. Docking analysis for 5 (PTP-1B).

Figure S71. Docking analysis for 6 (PTP-1B).

Figure S72. Docking analysis for 7 (PTP-1B).

Figure S73. Docking analysis for 9 (PTP-1B).

Figure S74. Docking analysis for 10 (PTP-1B).

Figure S75. Docking analysis for Ursolic Acid (PTP-1B).

Figure S76. Phylogram of the most likely tree $(-\operatorname{lnL}=2909.24)$ from a RAxML analysis of 39 strains from the ITS region (519 bp). Numbers refer to RAxML bootstrap support values $\geq 70 \%$ based on 1000 replicates. Malbranchea circinata (ATCC: 34526) is phylogenetically related to Myxotrichum spp. (Leotiomycetes incertae sedis, Myxotrichaceae, Ascomycota). 14-Day old cultures from Sabouraud dextrose agar; Difco, Corn meal agar; Difco, Malt extract agar; Difco, and Oatmeal agar; Difco is shown. Symbols "T, ET, and NT" denote taxa reported as type, ex-type, or neo-type specimens. Bar indicates nucleotide substitutions per site.

Figure S77. Phylogram of the most likely tree $(-\operatorname{lnL}=2091.26$ 10) from a RAxML analysis of 10 strains from the partial 28SrDNA; LSU region (871 bp). Numbers refer to RAxML bootstrap support values $\geq 70 \%$ based on 1000 replicates. Malbranchea circinata (ATCC: 34526) is identical to M. circinata, IFM4126 (AB35914) and sister to Myxotrichum deflexum, CBS 228.61 (MH869596) (Leotiomycetes incertae sedis, Myxotrichaceae, Ascomycota). Bar indicates nucleotide substitutions per site.

Figure S78. Conformations of compound 1 accounting for $100 \%$ of the conformational population.

Table S1. Geometry Optimization and NMR Single-Point Calculations at DFT B3LYP/6-311+G(2d,p)//M06-2X/6-31+g(d,p) Level of Theory for compound $\mathbf{1 .}$

Table S2. Geometry Optimization and NMR Single-Point Calculations at DFT B3LYP/6-311+G(2d,p)//M06-2X /6$31+\mathrm{g}(\mathrm{d}, \mathrm{p})$ Level of Theory for compound $\mathbf{2}$. 
Table S3. Shielding tensors and NMR Single-Point Calculations at DFT B3LYP/6-311+G(2d,p)// M06-2X /6-31+g(d,p) Level of Theory for compound 1.

Table S4. Shielding tensors and NMR Single-Point Calculations at DFT B3LYP/6-311+G(2d,p)/ M06-2X/6-31+g(d,p) Level of Theory for compound 2.

Table S5. Inhibitory Effect of Compounds 1-12 Tested against Yeast $\alpha$-Glucosidase ( $\alpha \mathrm{GHY}$ ) and Protein Tyrosine Phosphatase 1B (PTP-1B)

Additional Experimental Details and Supporting References 


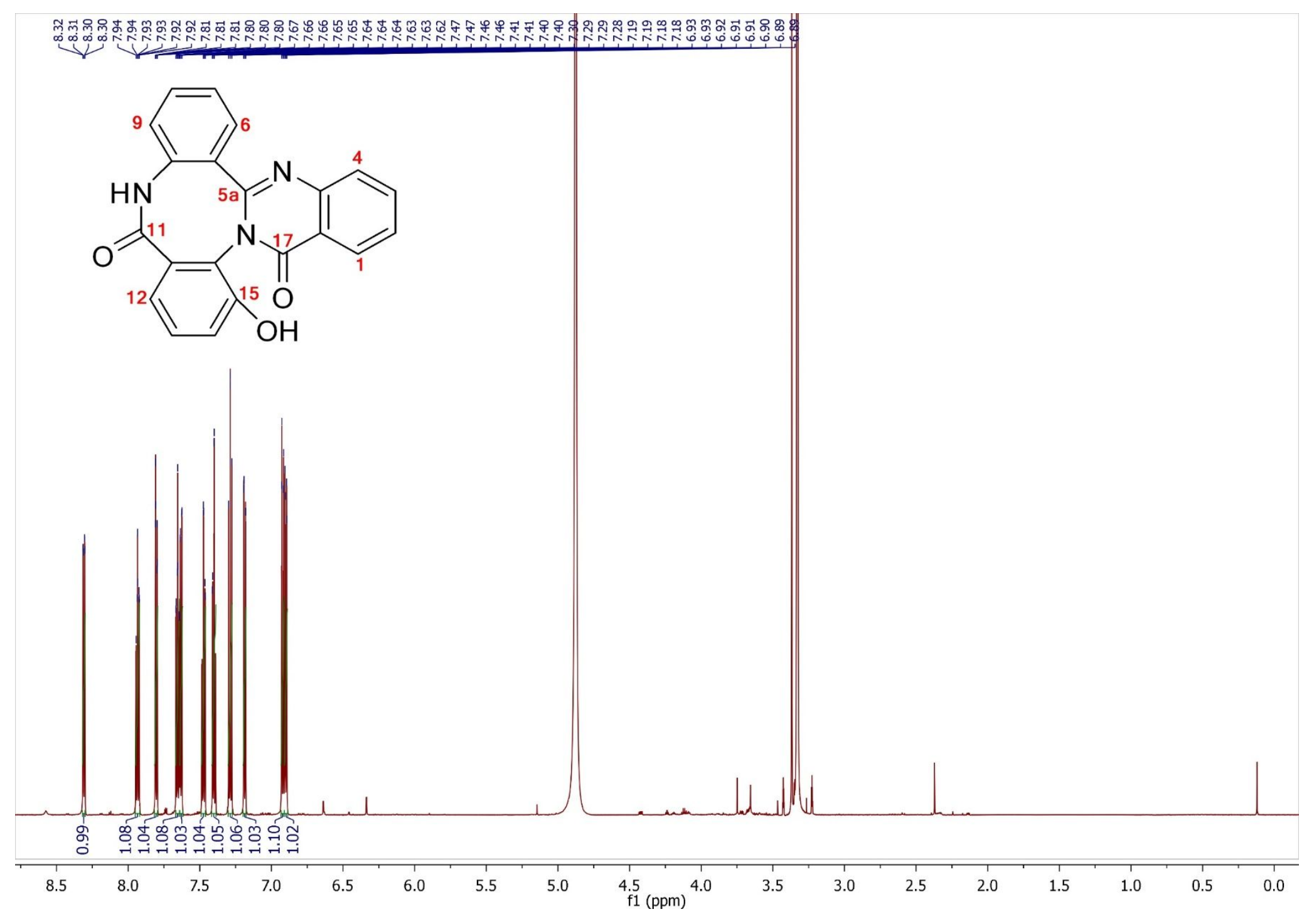

Figure S1. ${ }^{1} \mathrm{H}$ NMR spectrum of 1 in $\mathrm{CD}_{3} \mathrm{OD}(700 \mathrm{MHz})$. 


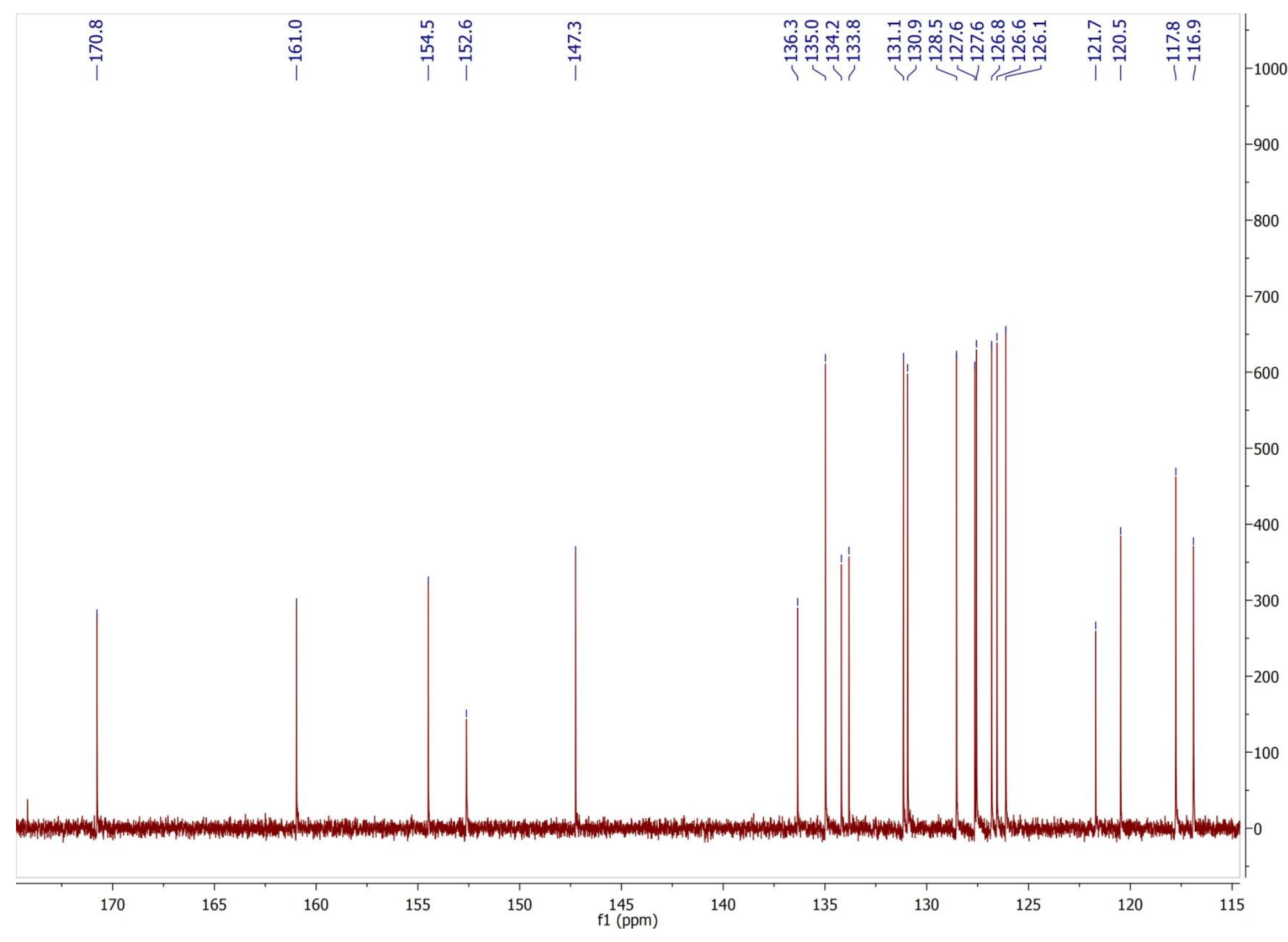

Figure S2. ${ }^{13} \mathrm{C}$ NMR spectrum of $\mathbf{1}$ in $\mathrm{CD}_{3} \mathrm{OD}(175 \mathrm{MHz})$. 


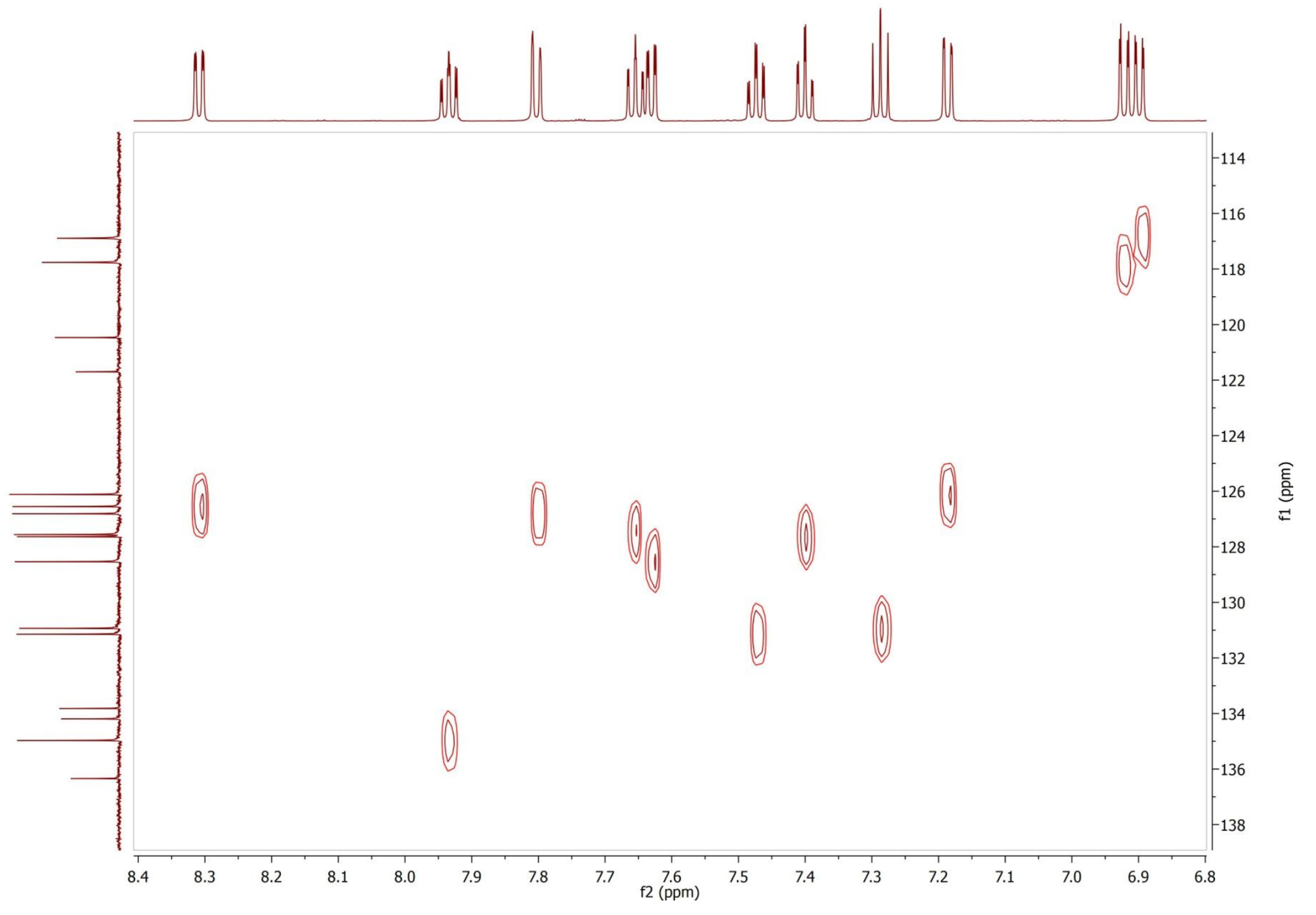

Figure S3. HSQC spectrum of $\mathbf{1}$ in $\mathrm{CD}_{3} \mathrm{OD}$. 


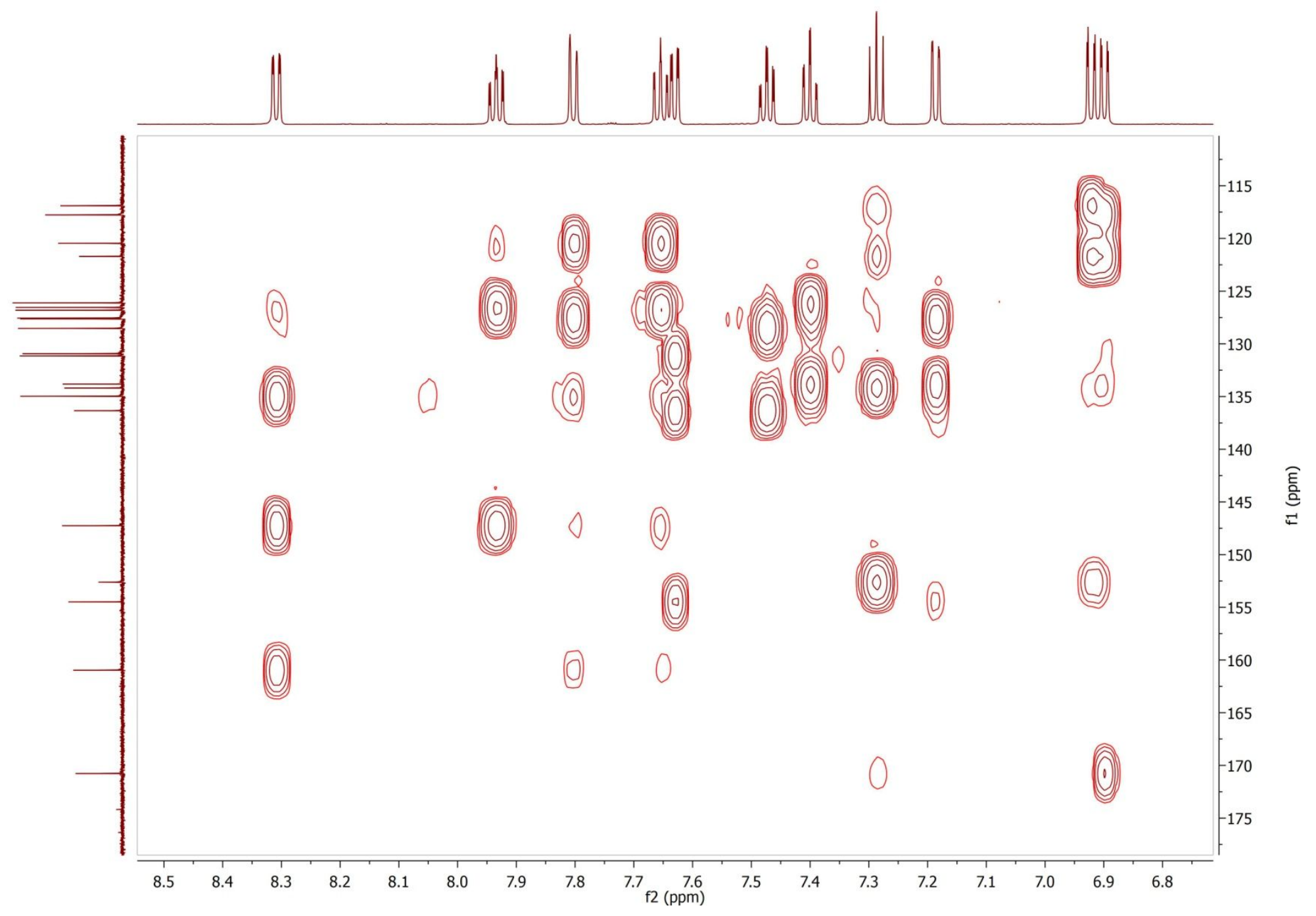

Figure S4. $\mathrm{HMBC}$ spectrum of $\mathbf{1}$ in $\mathrm{CD}_{3} \mathrm{OD}$. 


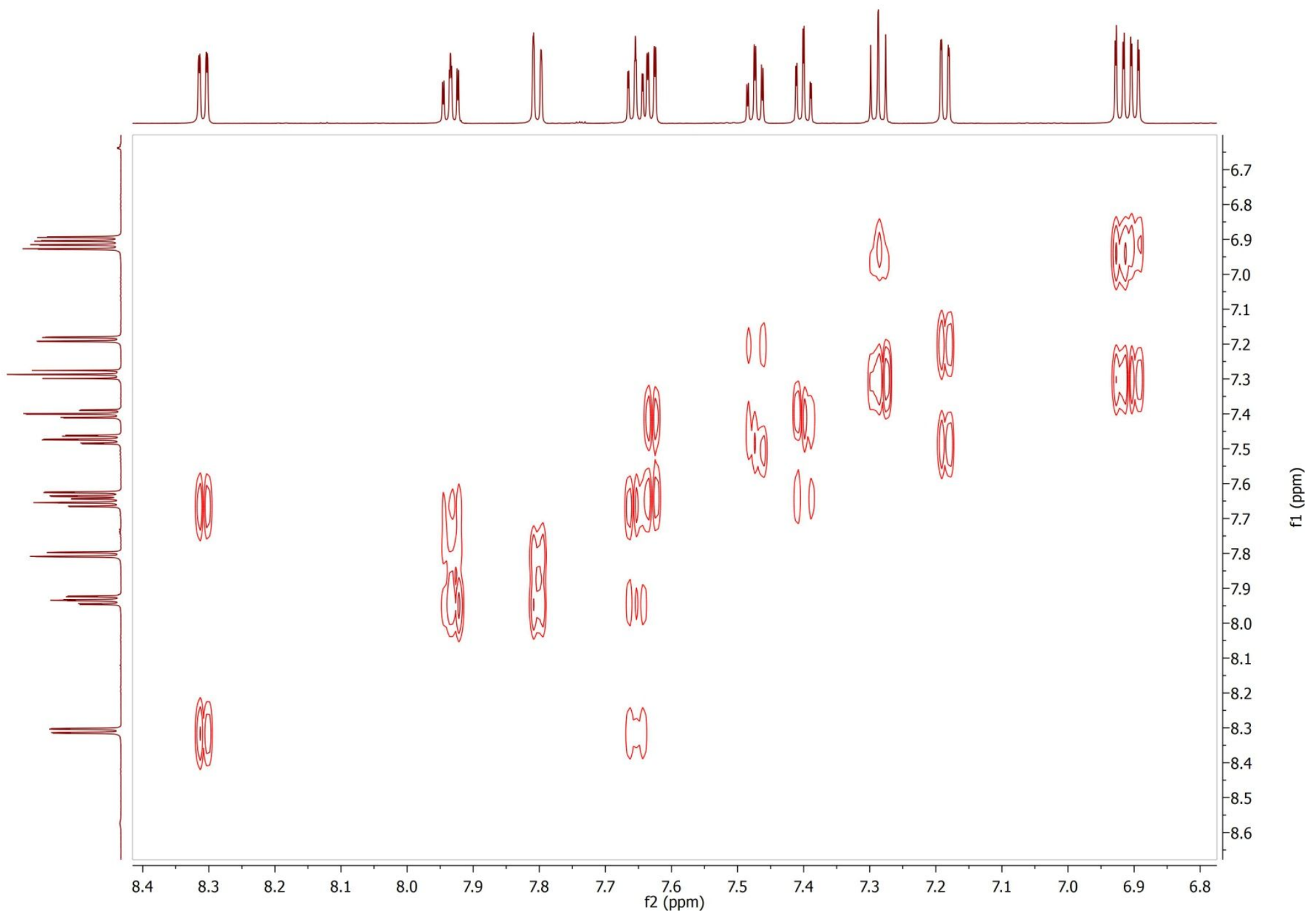

Figure S5. COSY spectrum of $\mathbf{1}$ in $\mathrm{CD}_{3} \mathrm{OD}$. 


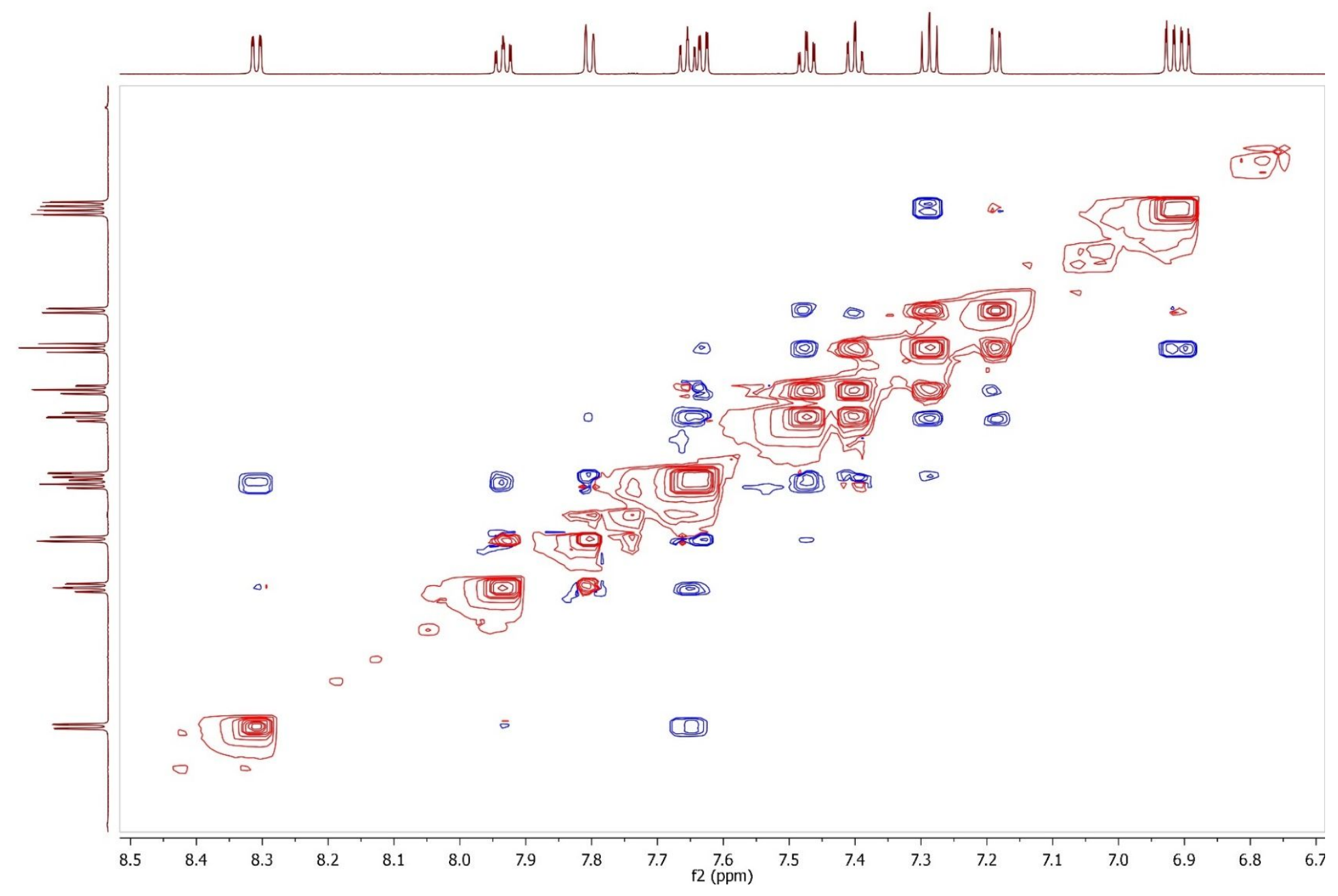

Figure S6. NOESY spectrum of $\mathbf{1}$ in $\mathrm{CD}_{3} \mathrm{OD}$. 
INSTITUTO DE QUIMICA, UNAM

LABORATORIO DE ESPECTROMETRIA DE MASAS

Data:2346 MR14-C5

Sample Name:Dra Macias Martha- Operador: Carmen Gar cia

Description:

lonization Mode:ESI+

2:45:32 PM

Operator:AccuTOF

Mass Calibration data:Cal Peg 600

History:Determine m/z[Peak Detect[Centroid,30,Area] ;Correct Base[1.0\%]];Correct Base[5.0\%];Average(MS[1] 1..1)

Created:11/8/2018 7:41:48 AM

Charge number: 1

Element: ${ }^{12} \mathrm{C}: 0$.. 30, ${ }^{1} \mathrm{H}: 0 . .30,{ }^{14} \mathrm{~N}: 0$.. $3,{ }^{16} \mathrm{O}: 0$.. 3

Tolerance:3.00(mmu)

Unsaturation Number:0.0 .. 20.0 (Fraction:Both)

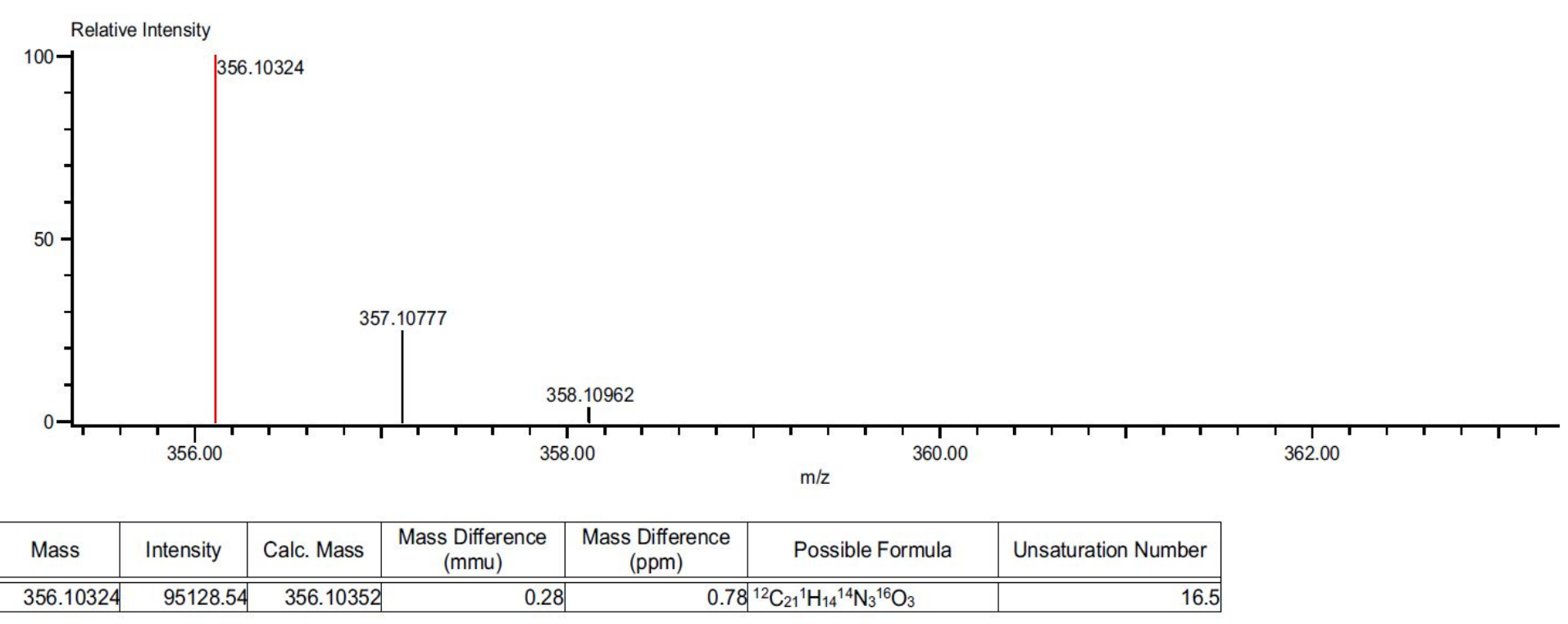

Figure S7. HRESIMS spectrum of $\mathbf{1}$. 


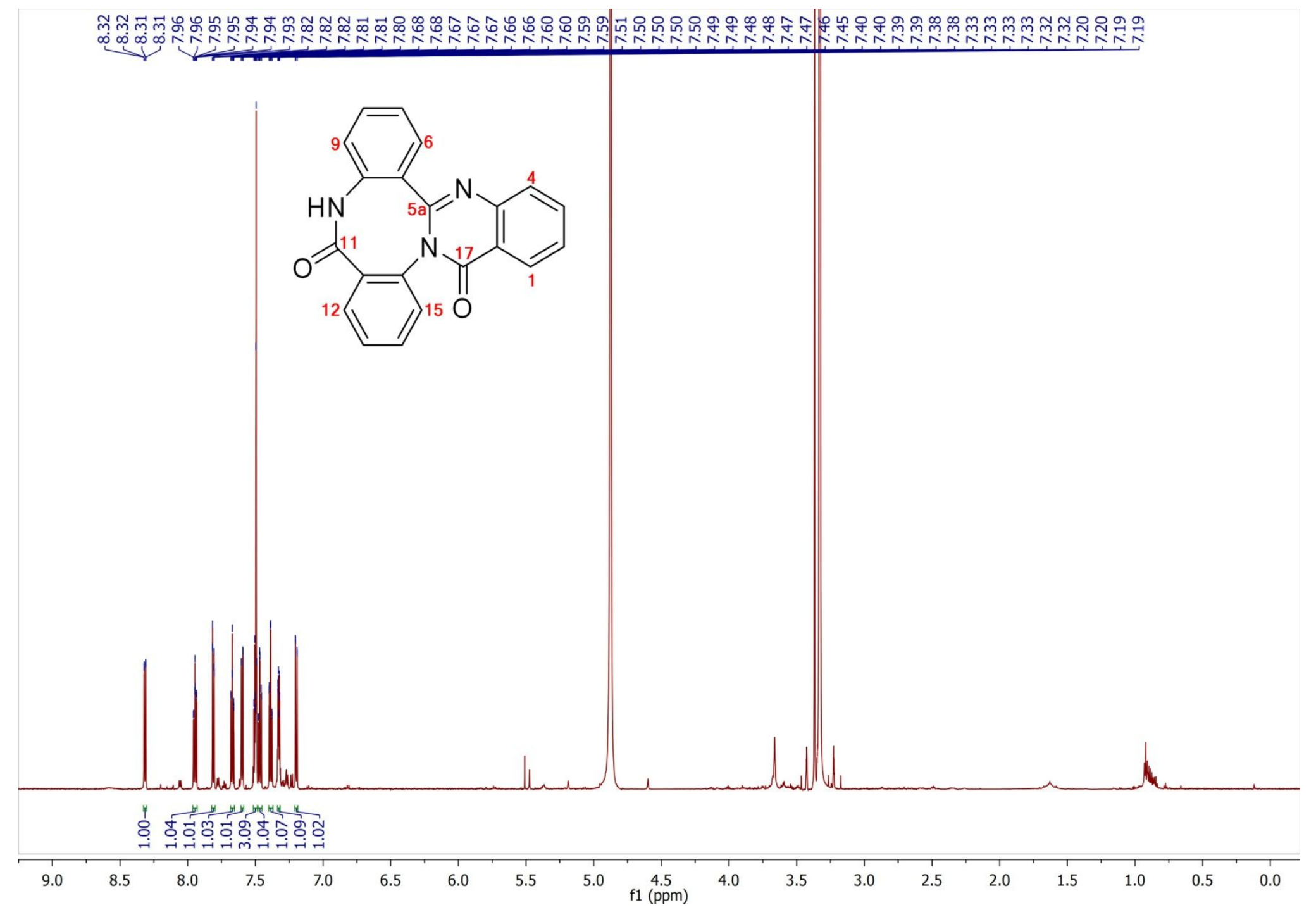

Figure S8. ${ }^{1} \mathrm{H}$ NMR spectrum of 2 in $\mathrm{CD}_{3} \mathrm{OD}(700 \mathrm{MHz})$. 


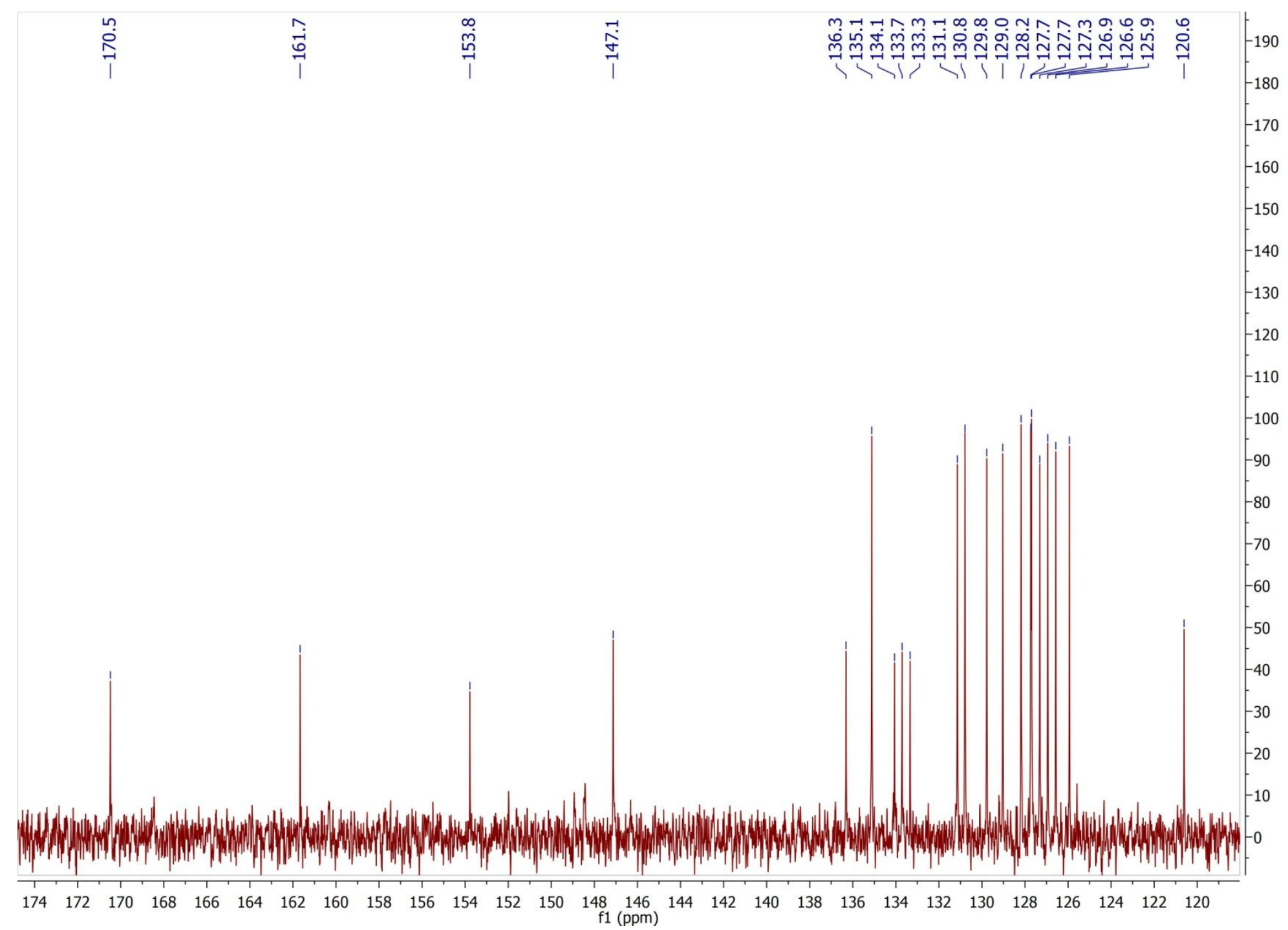

Figure S9. ${ }^{13} \mathrm{C}$ NMR spectrum of 2 in $\mathrm{CD}_{3} \mathrm{OD}(175 \mathrm{MHz})$. 


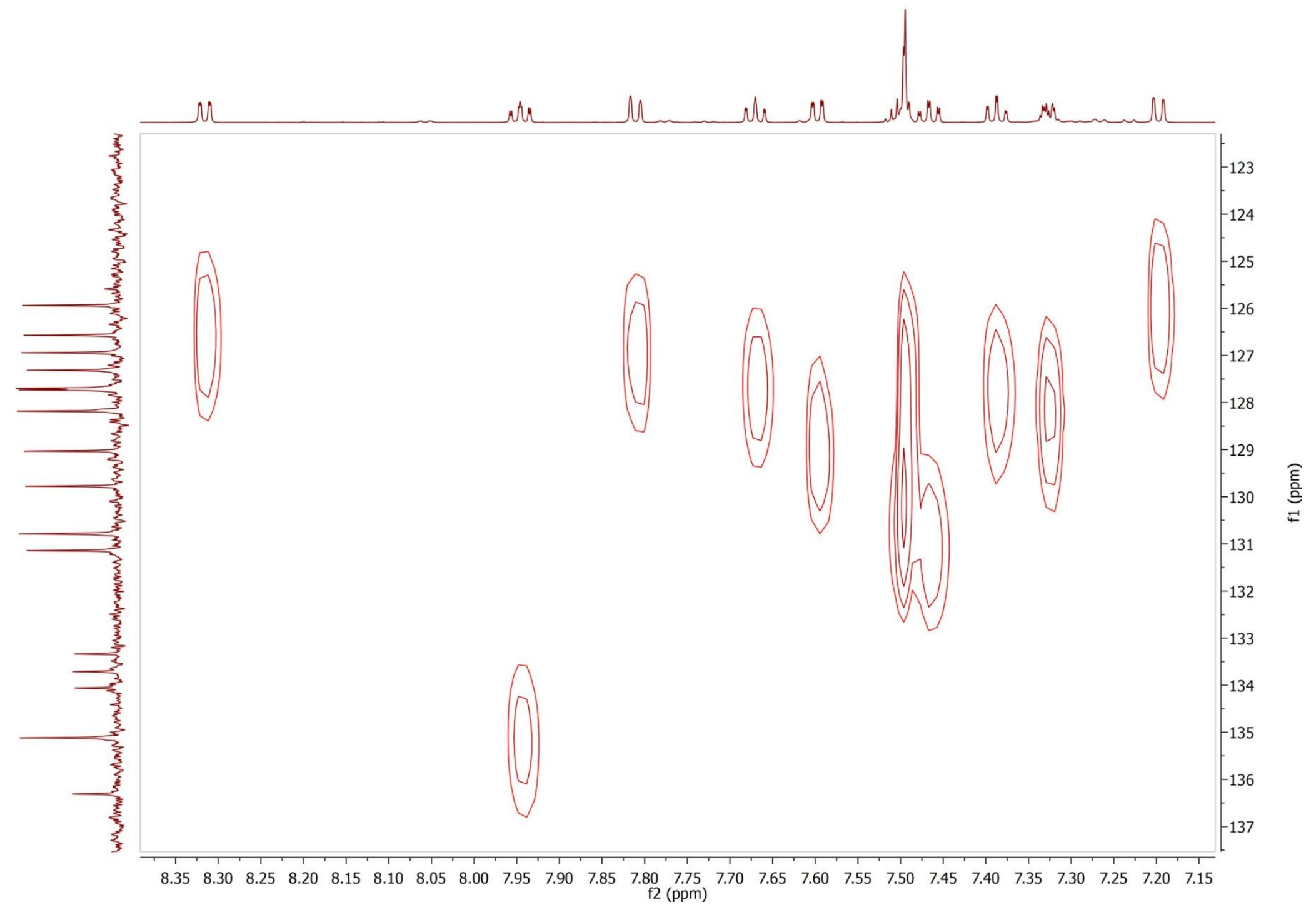

Figure S10. HSQC spectrum of $\mathbf{2}$ in $\mathrm{CD}_{3} \mathrm{OD}$. 


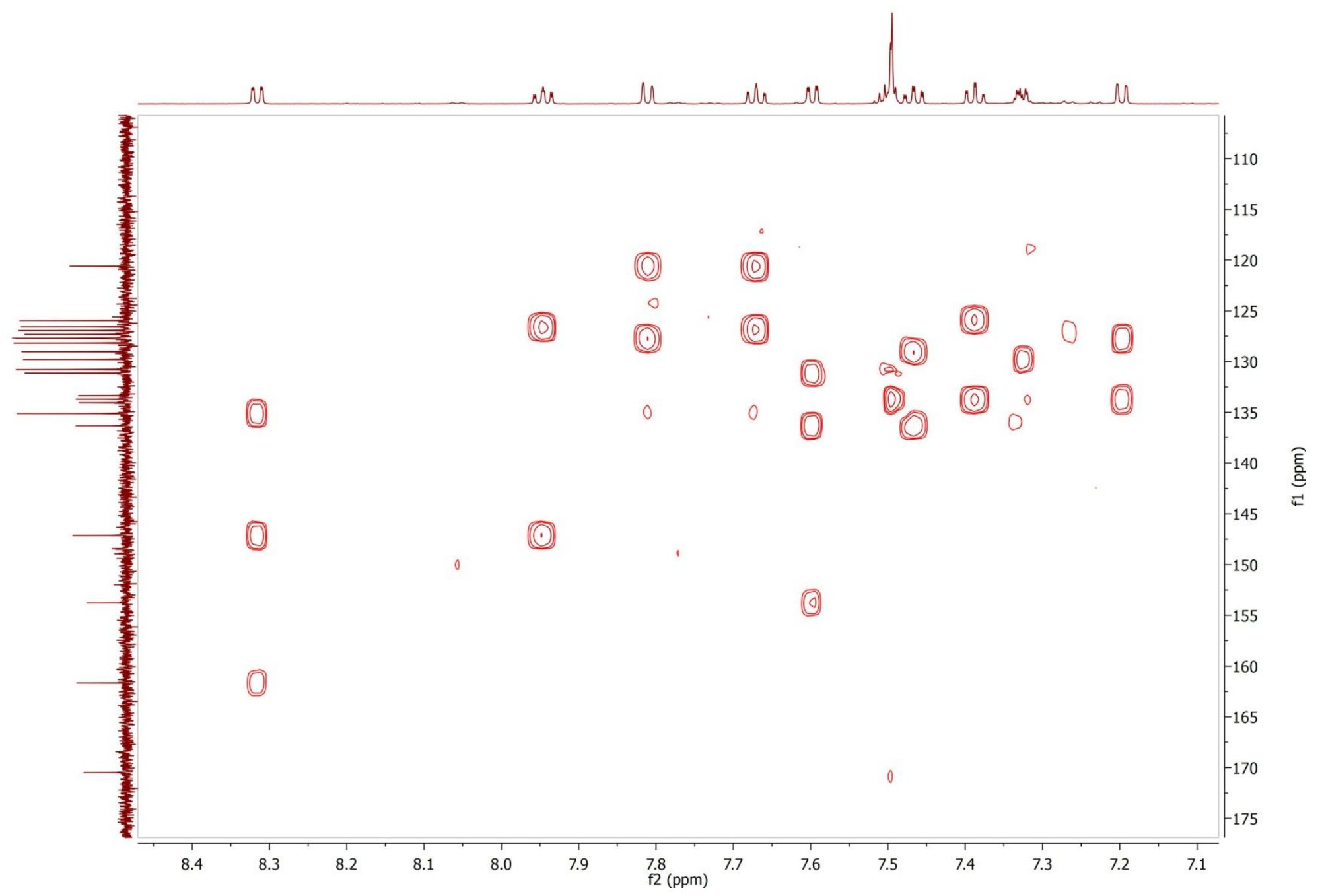

Figure S11. HMBC spectrum of 2 in $\mathrm{CD}_{3} \mathrm{OD}$. 


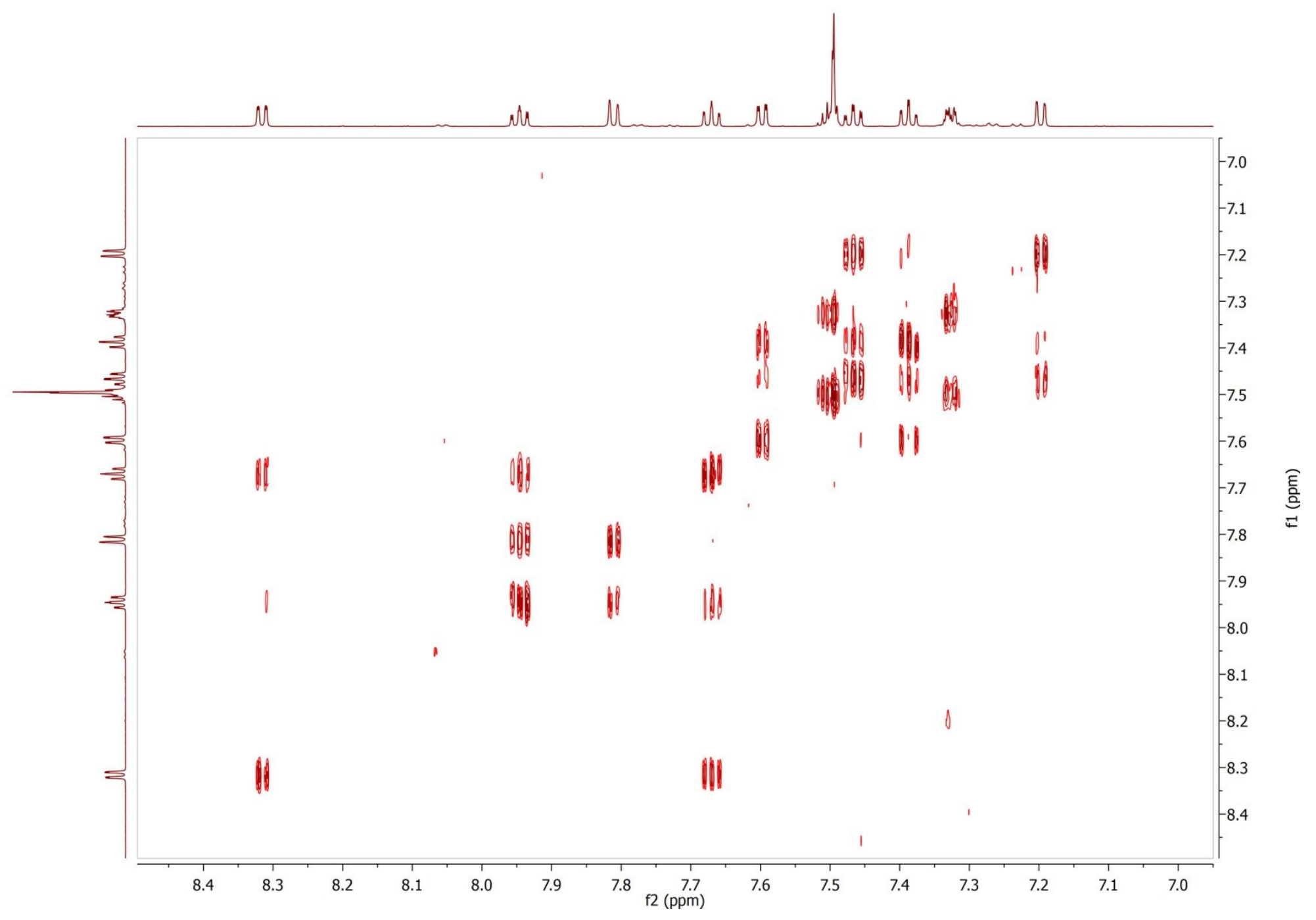

Figure S12. COSY spectrum of $\mathbf{2}$ in $\mathrm{CD}_{3} \mathrm{OD}$. 


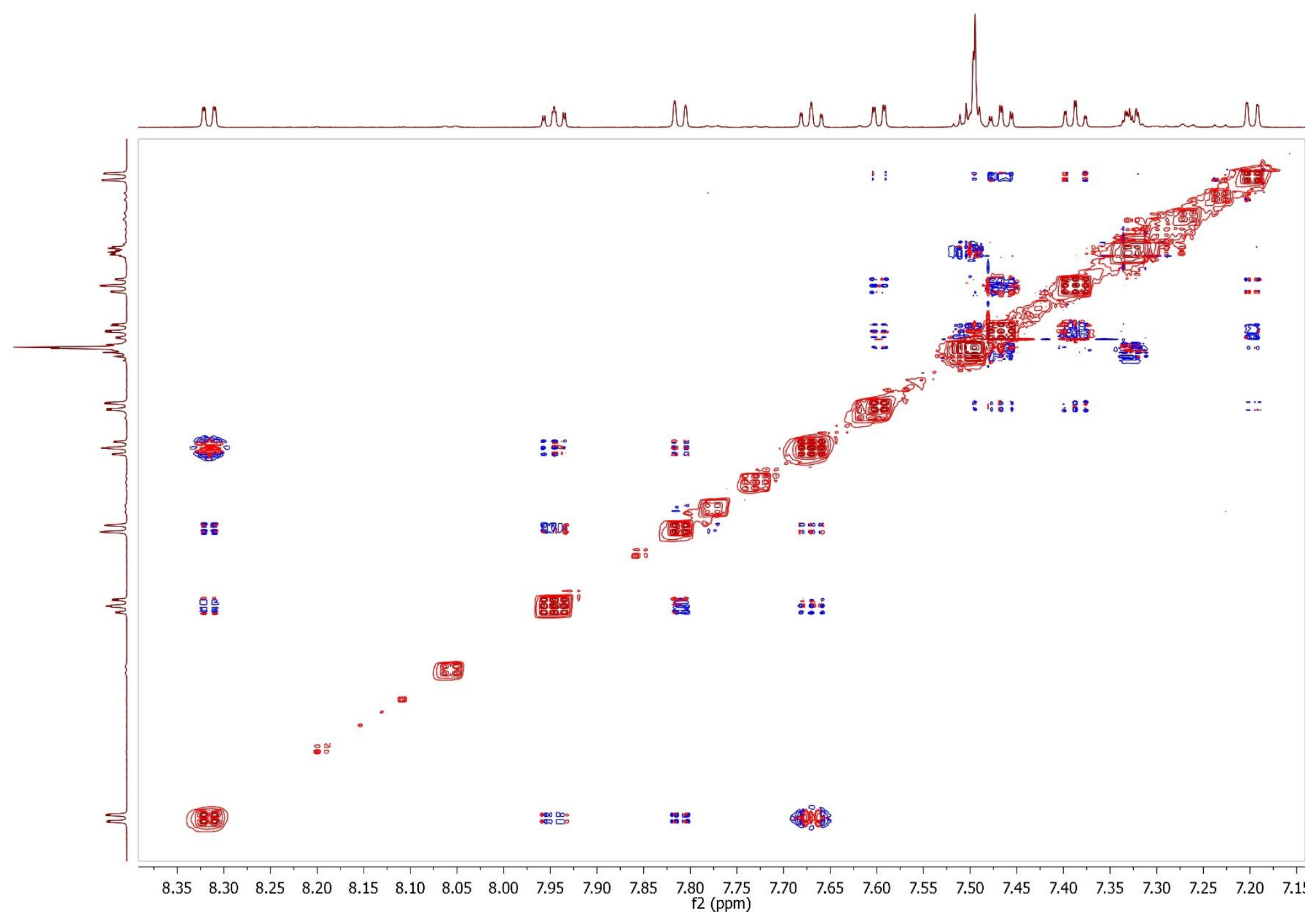

Figure S13. NOESY spectrum of $\mathbf{2}$ in $\mathrm{CD}_{3} \mathrm{OD}$. 


\section{INSTITUTO DE QUIMICA, UNAM}

LABORATORIO DE ESPECTROMETRIA DE MASAS

Data:2345 MR14-A5

Sample Name:Dra Macias Martha- Operador: Carmen Garcia

Description:

Acquired:10/29/2018 3:41:26 PM

Operator:AccuTOF

Mass Calibration data:Cal Peg 600

Created:11/9/2018 7:41:48 AM

lonization Mode:ESI+

Created by:AccuTOF

Charge number: 1

Element: ${ }^{12} \mathrm{C}: 0$.. 30, ${ }^{1} \mathrm{H}: 0$.. 30, ${ }^{14} \mathrm{~N}: 0$.. 3, ${ }^{16} \mathrm{O}: 0$.. 2

Tolerance: $3.00(\mathrm{mmu})$

Unsaturation Number:0.0 .. 20.0 (Fraction:Both)

Relative Intensity

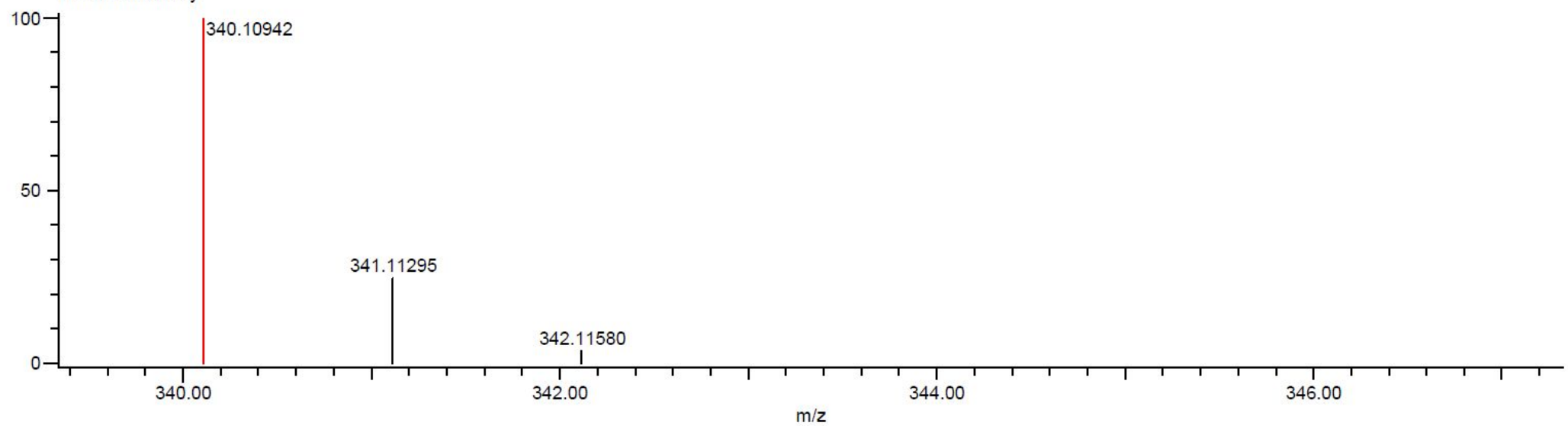

\begin{tabular}{|c|c|c|c|c|c|c|}
\hline Mass & Intensity & Calc. Mass & $\begin{array}{c}\text { Mass Difference } \\
(\mathrm{mmu})\end{array}$ & $\begin{array}{c}\text { Mass Difference } \\
(\mathrm{ppm})\end{array}$ & Possible Formula & Unsaturation Number \\
\hline \hline 340.10942 & 181128.63 & 340.10860 & 0.82 & 2.40 & ${ }^{12} \mathrm{C}_{21}{ }^{1} \mathrm{H}_{14}{ }^{14} \mathrm{~N}_{3}{ }^{16} \mathrm{O}_{2}$ & 16.5 \\
\hline
\end{tabular}

Figure S14. HRESIMS spectrum of 2. 


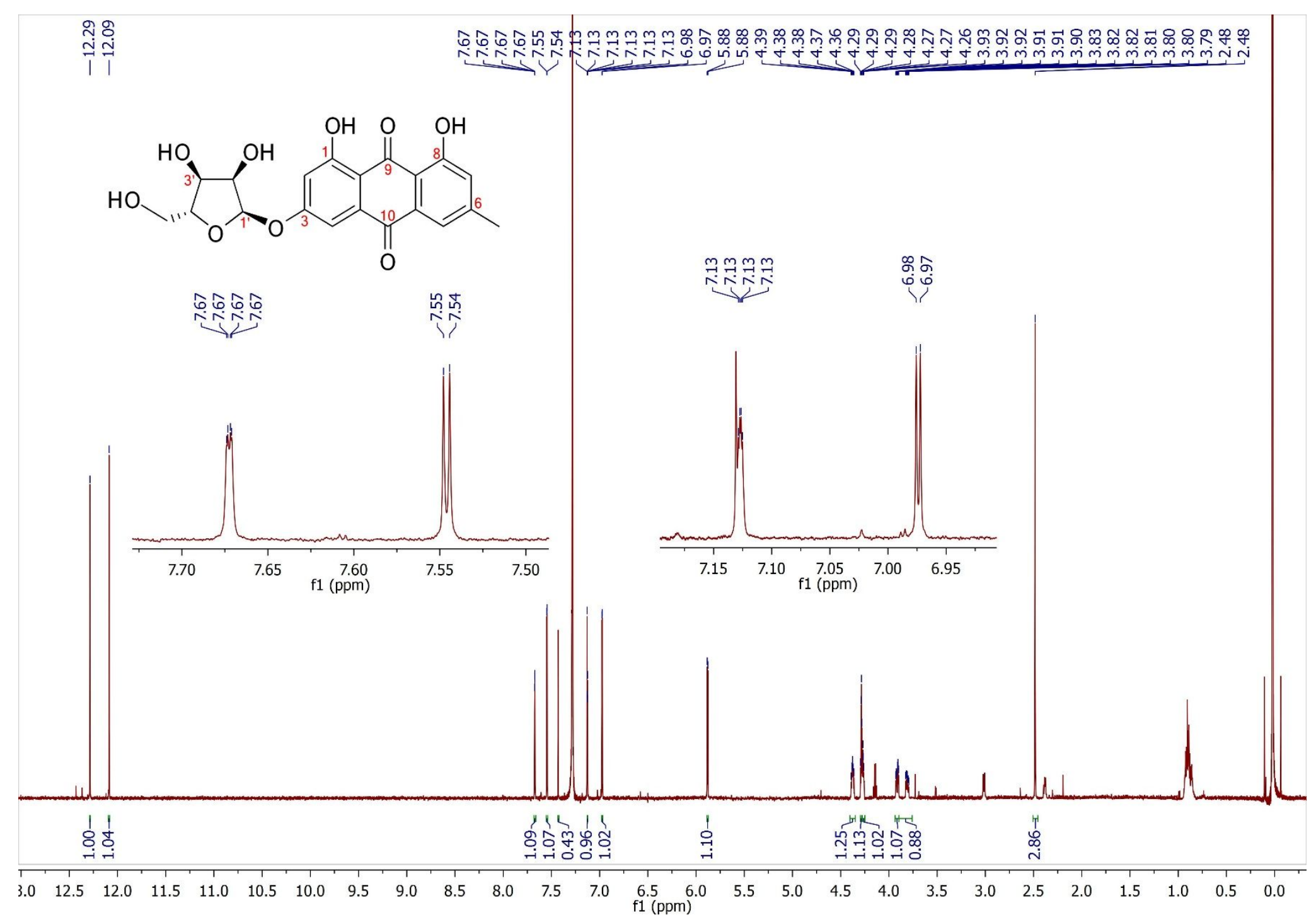

Figure S15. ${ }^{1} \mathrm{H}$ NMR spectrum of 3 in $\mathrm{CDCl}_{3}(700 \mathrm{MHz})$. 


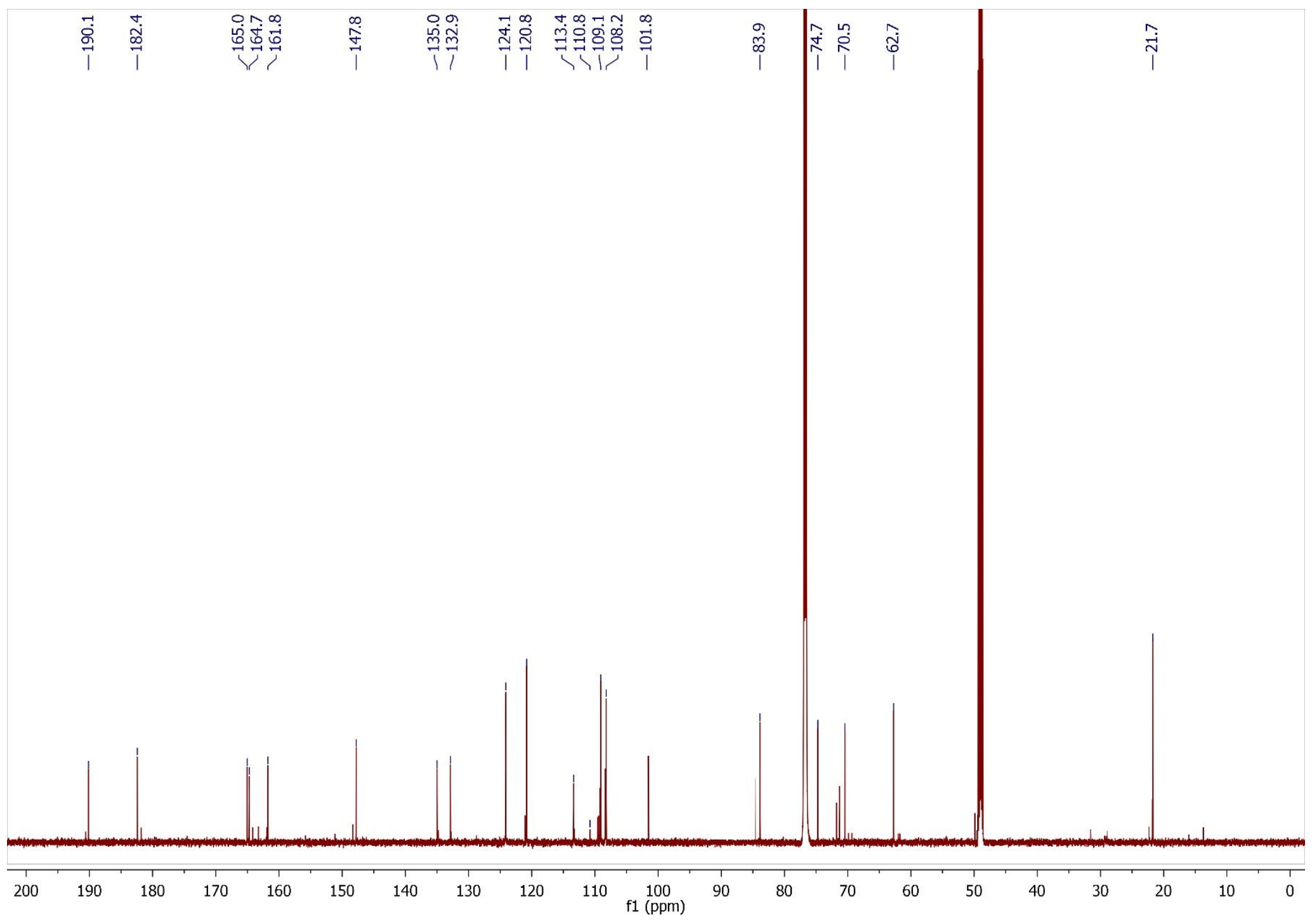

Figure S16. ${ }^{13} \mathrm{C}$ NMR spectrum of 3 in $\mathrm{CDCl}_{3}+$ drops of $\mathrm{CD}_{3} \mathrm{OD}(175 \mathrm{MHz})$.

19 


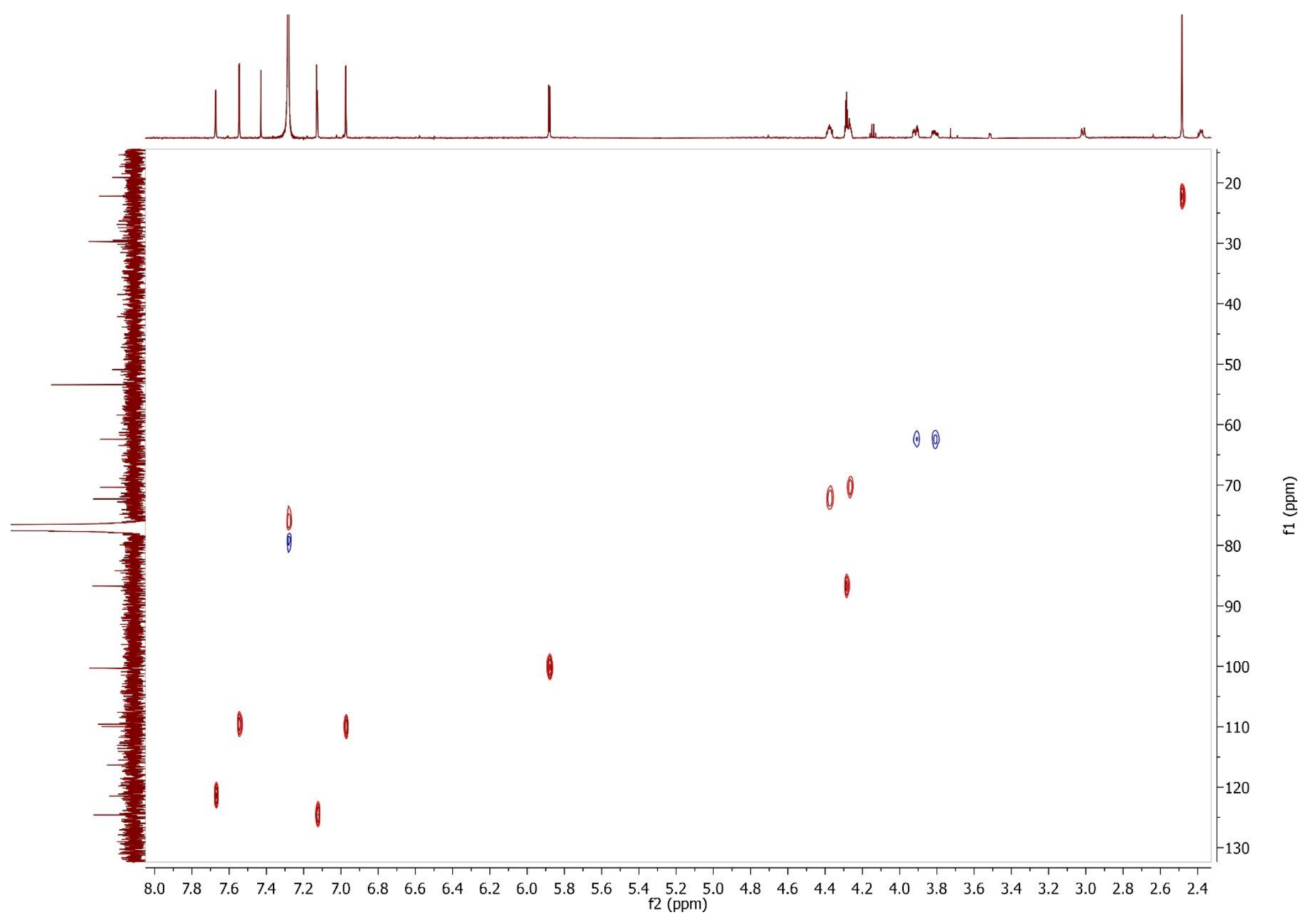

Figure S17. HSQC spectrum of 3 in $\mathrm{CDCl}_{3}+$ drops of $\mathrm{CD}_{3} \mathrm{OD}$. 


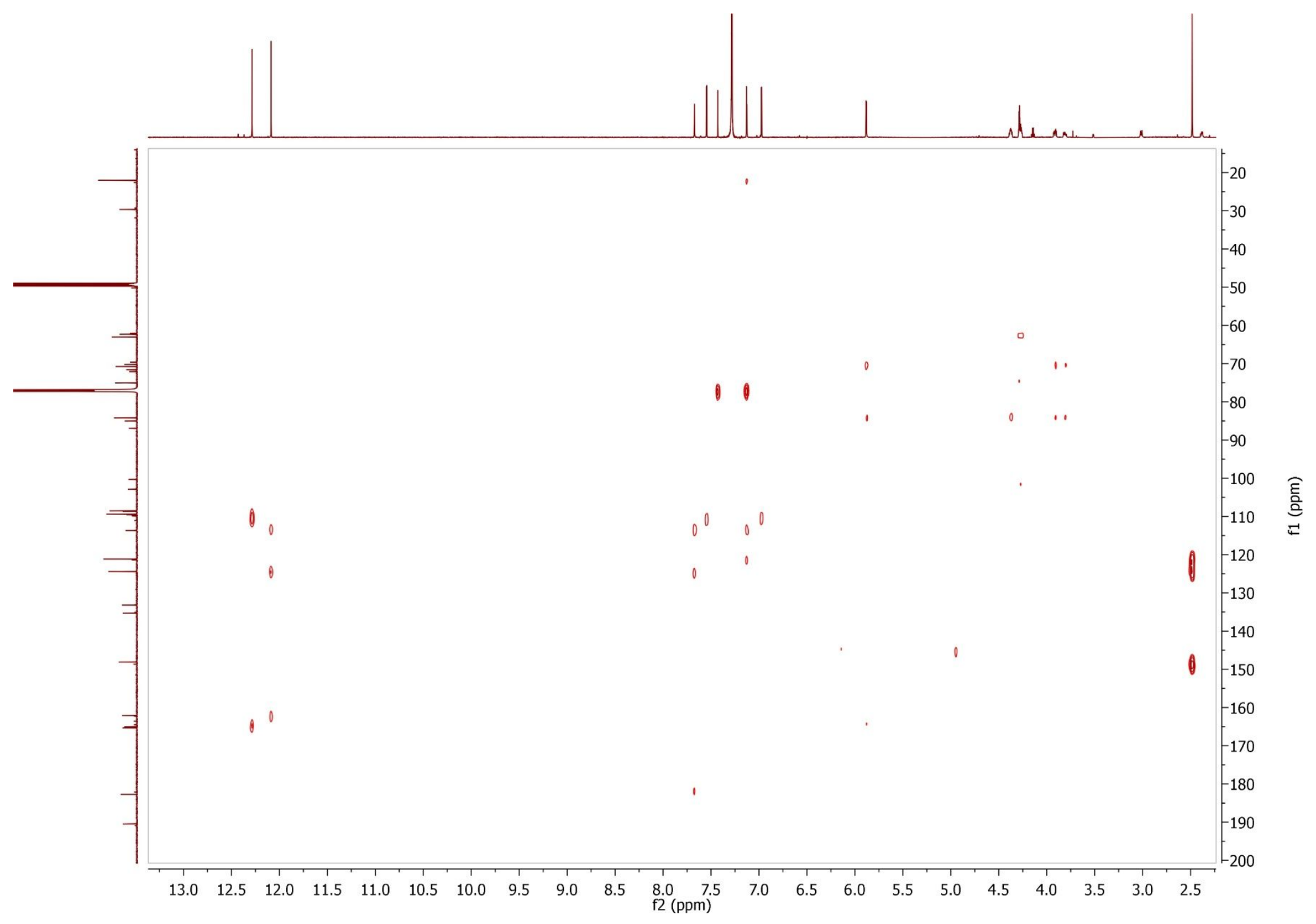

Figure S18. $\mathrm{HMBC}$ spectrum of $\mathbf{3}$ in $\mathrm{CDCl}_{3}+$ drops of $\mathrm{CD}_{3} \mathrm{OD}$. 


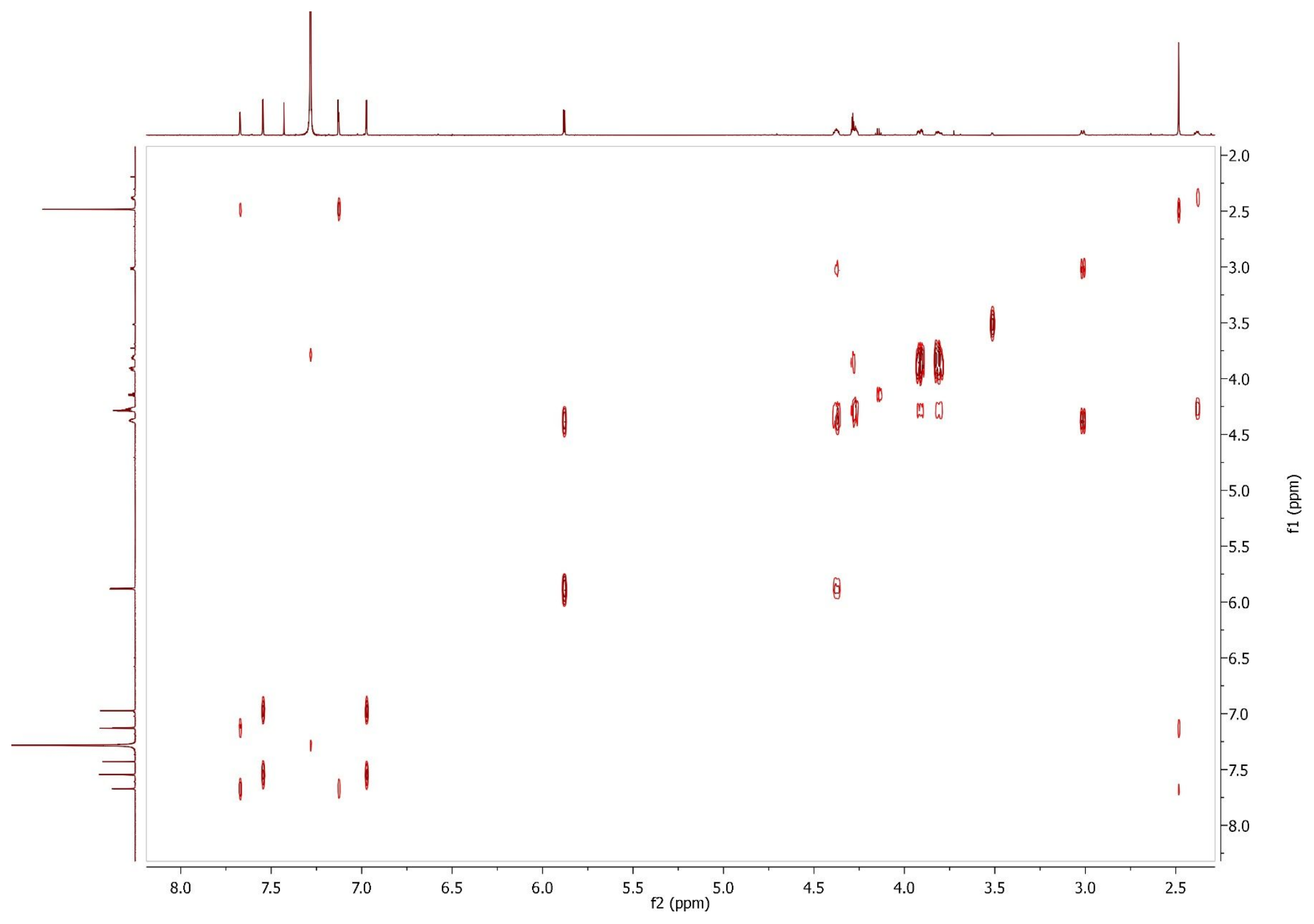

Figure S19. COSY spectrum of 3 in $\mathrm{CDCl}_{3}+$ drops of $\mathrm{CD}_{3} \mathrm{OD}$. 


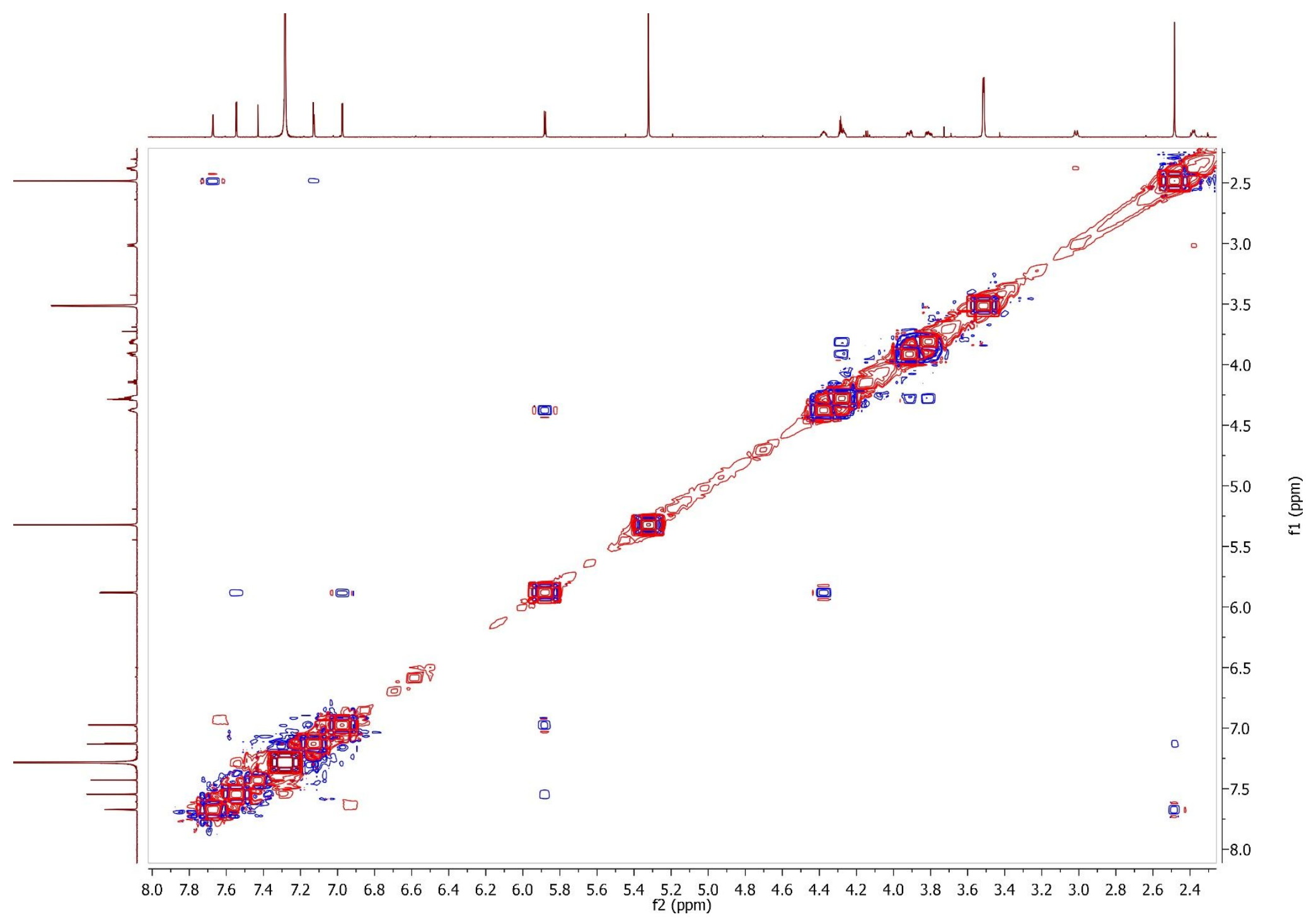

Figure S20. NOESY spectrum of $\mathbf{3}$ in $\mathrm{CDCl}_{3}+$ drops of $\mathrm{CD}_{3} \mathrm{OD}$. 
INSTITUTO DE QUIMICA, UNAM

LABORATORIO DE ESPECTROMETRIA DE MASAS

\section{Data.924 MR15-Ma-C5}

Sample Name:Dra Macias Martha / Operador: Carmen G arcia-Javier Perez

Description:

Acquired:5/22/2019 11:55:21 AM Operator:AccuTOF

Mass Calibration data:Cal Peg 600

onization Mode:ESI+

Created:5/31/2019 12:35:24 PM

History:Determine m/z[Peak Detect[Centroid,30,Area];Correct Base[];Smooth[5]];Correct Base[5.0\%];Avera ge(MS[... Created by:AccuTOF

Charge number: 1

Element: ${ }^{12} \mathrm{C}: 0$.. 30, ${ }^{1} \mathrm{H}: 0$.. 50, ${ }^{16} \mathrm{O}: 0$.. 15

Tolerance:5.00(mmu)

Unsaturation Number:0.0 .. 50.0 (Fraction:Both)

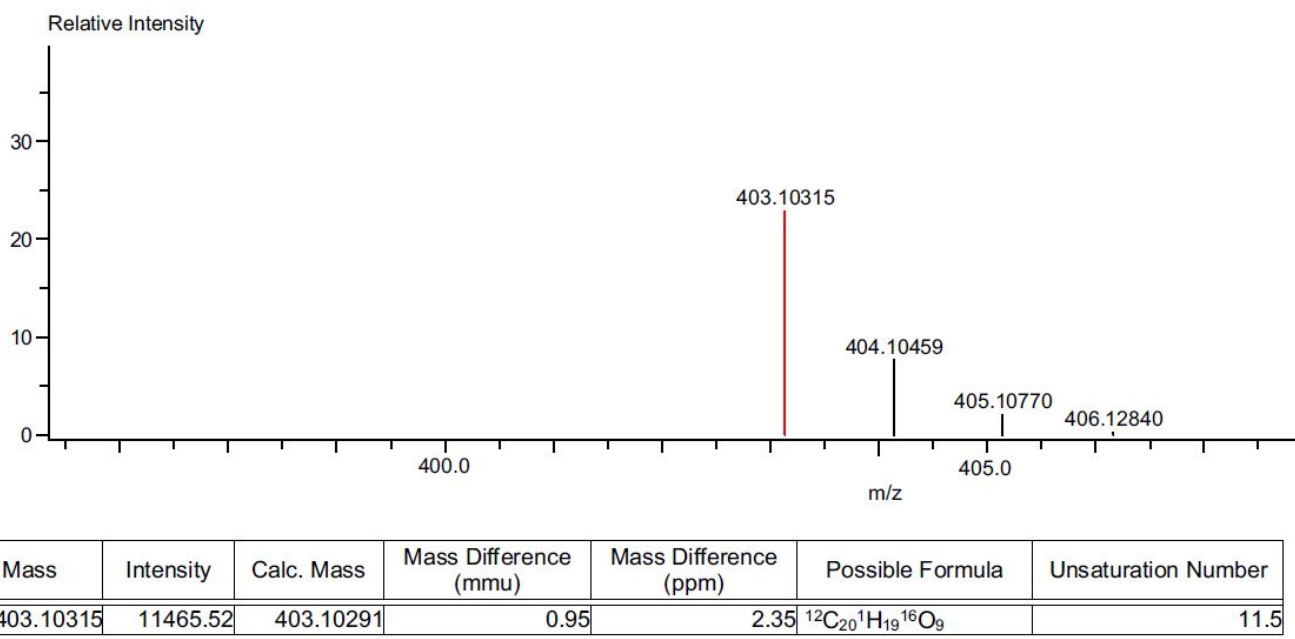

Figure S21. HRESIMS spectrum of $\mathbf{3}$. 


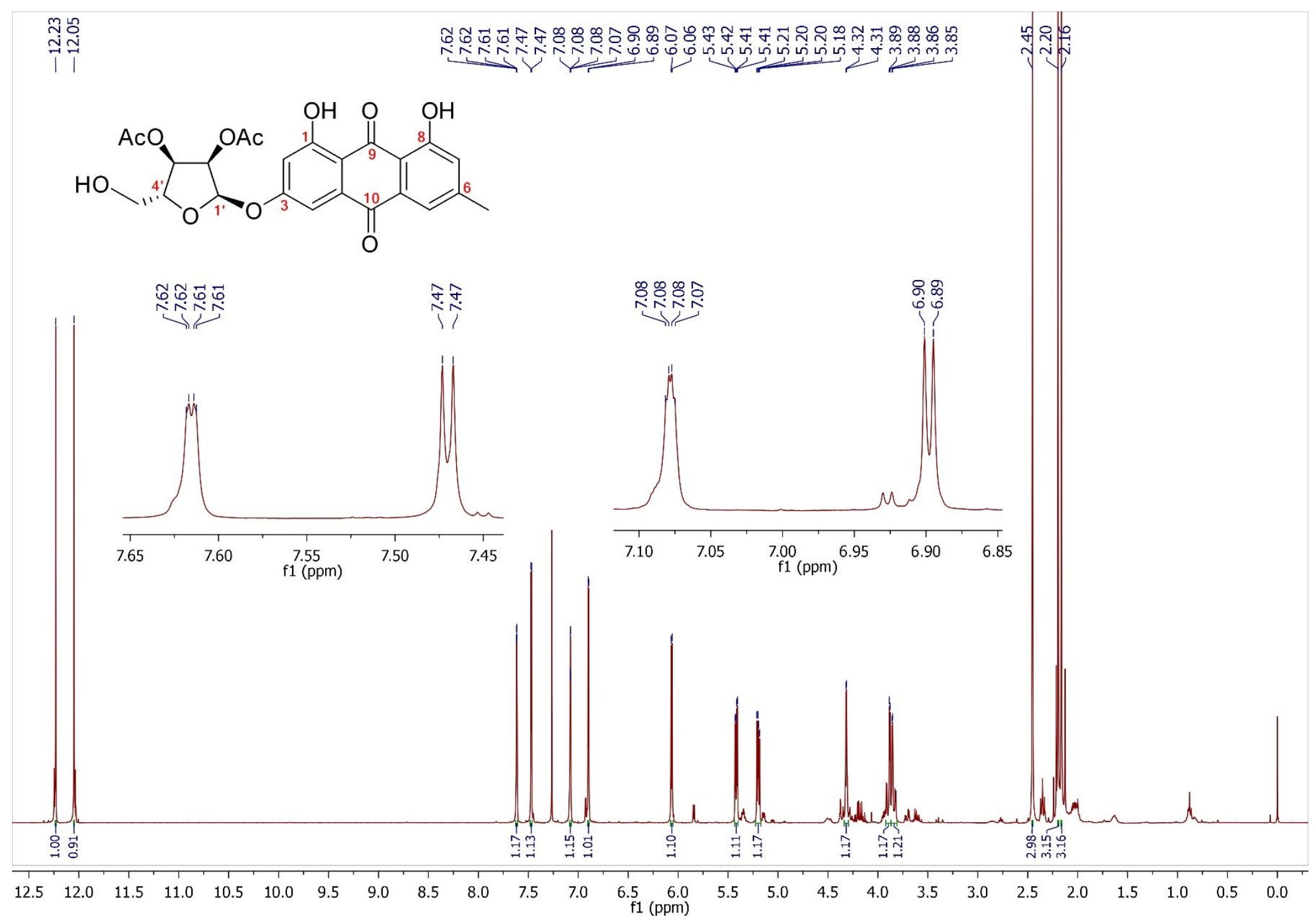

Figure S22. ${ }^{1} \mathrm{H}$ NMR spectrum of 4 in $\mathrm{CDCl}_{3}(400 \mathrm{MHz})$. 


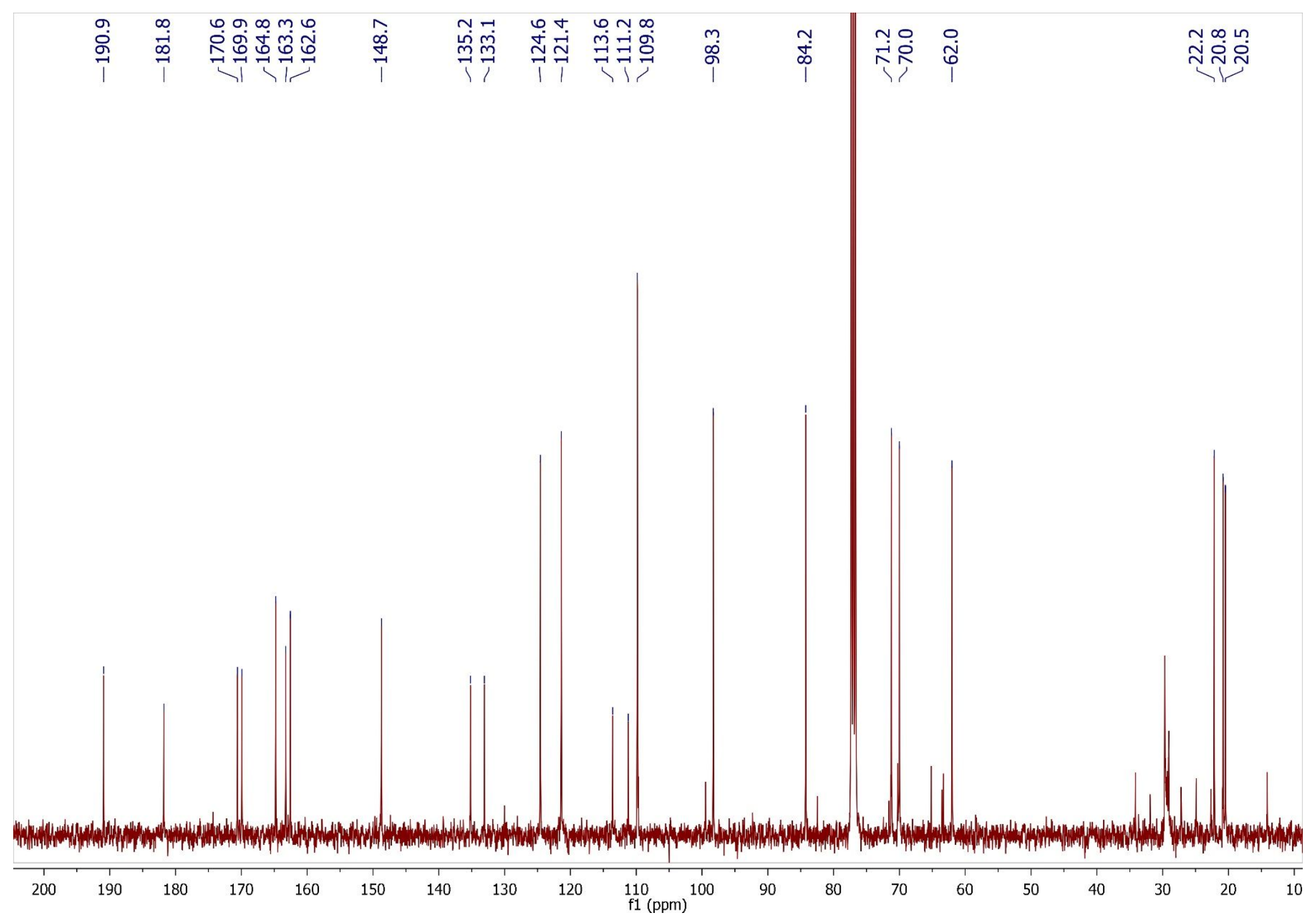

Figure S23. ${ }^{13} \mathrm{C}$ NMR spectrum of 4 in $\mathrm{CDCl}_{3}(100 \mathrm{MHz})$. 


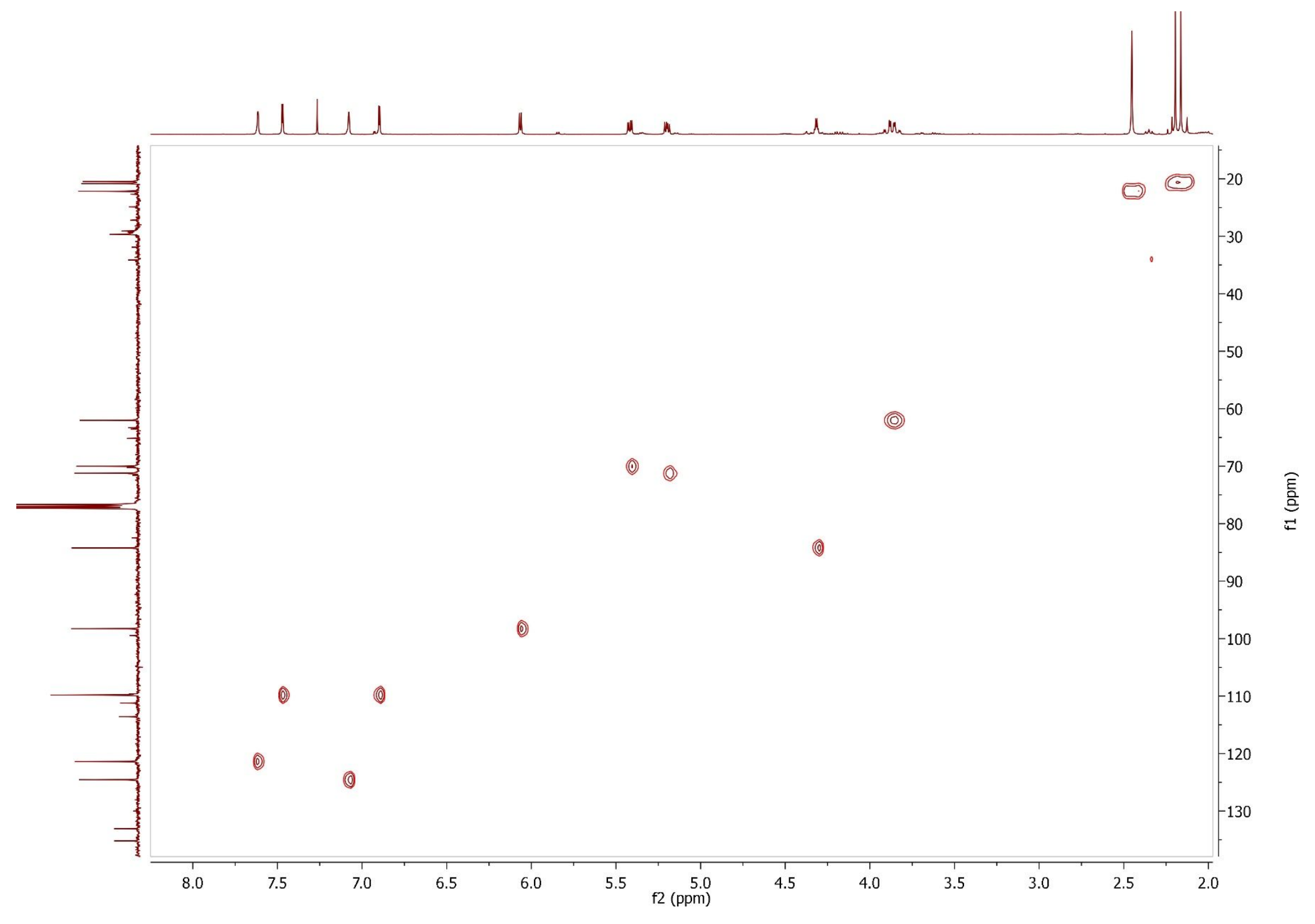

Figure S24. HSQC spectrum of $\mathbf{4}$ in $\mathrm{CDCl}_{3}$. 


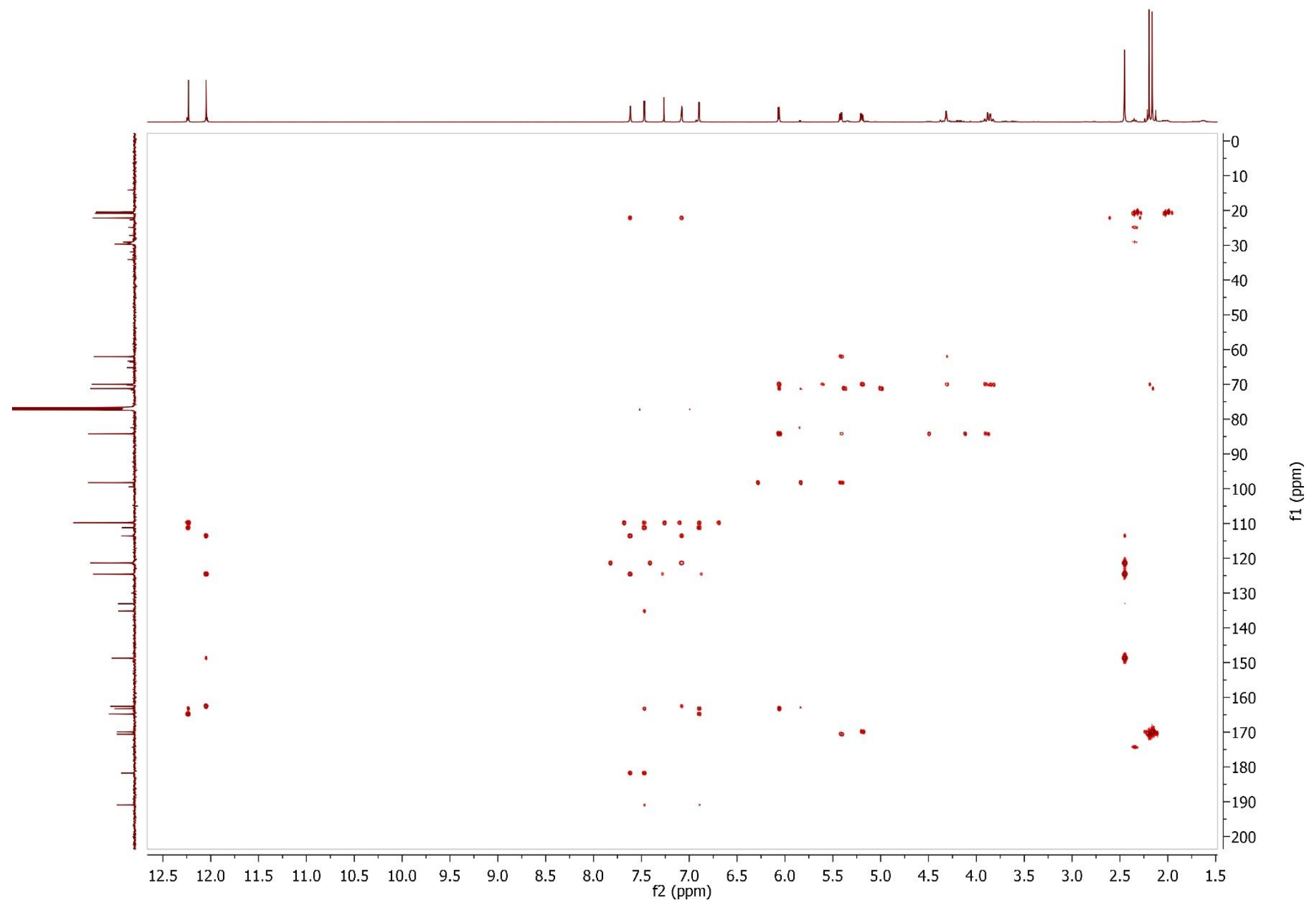

Figure S25. $\mathrm{HMBC}$ spectrum of $\mathbf{4}$ in $\mathrm{CDCl}_{3}$. 


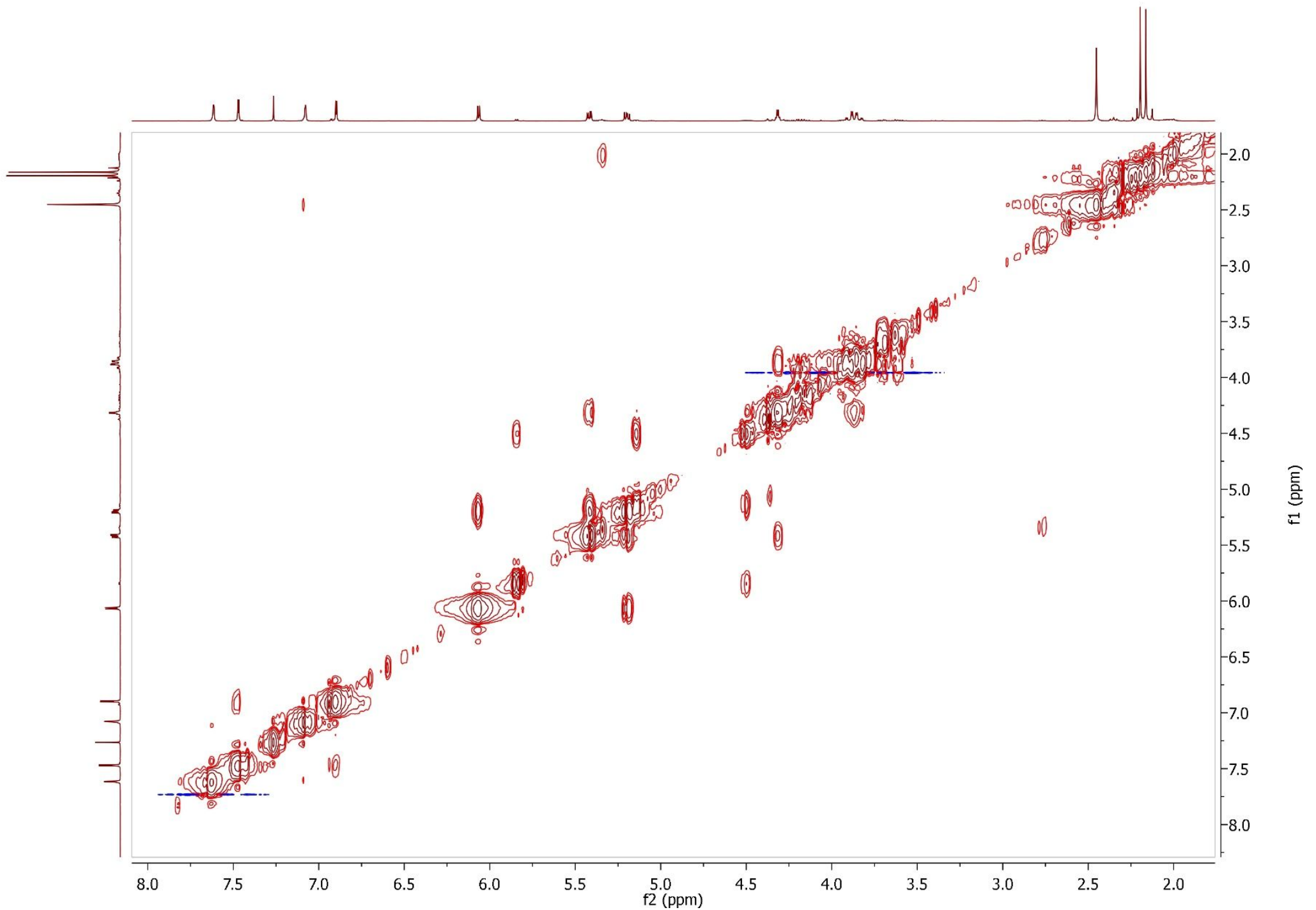

Figure S26. COSY spectrum of 4 in $\mathrm{CDCl}_{3}$. 


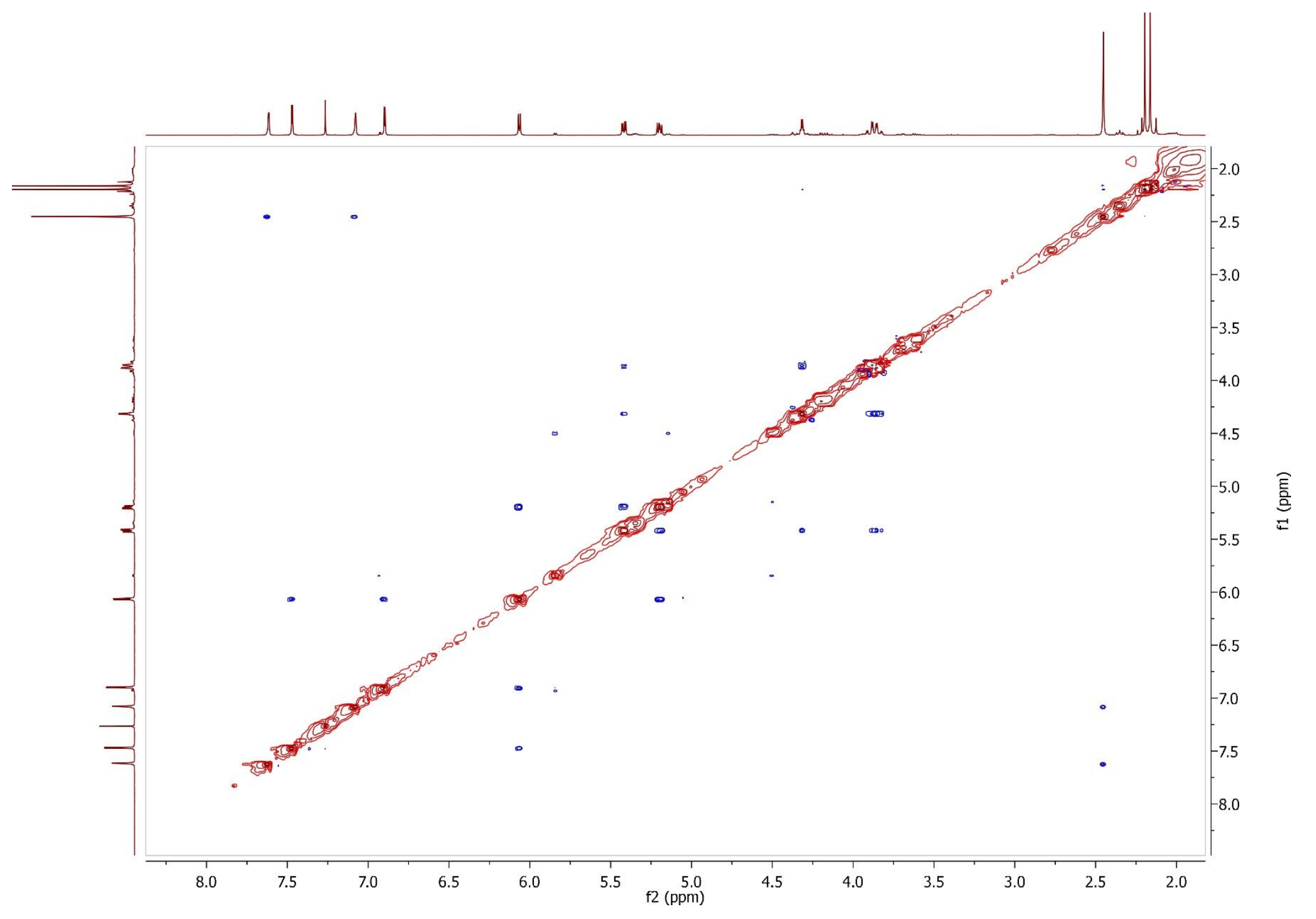

Figure S27. NOESY spectrum of $\mathbf{4}$ in $\mathrm{CDCl}_{3}$. 


\section{INSTITUTO DE QUIMICA, UNAM}

\section{LABORATORIO DE ESPECTROMETRIA DE MASAS}

Data:1984 MR14-F

Sample Name:Dra Macias Martha- Operador: Carmen Gar cia

Acquired:10/29/2018 12:35:28 PM

Description:

Operator:AccuTOF

Mass Calibration data:Cal Peg 600

lonization Mode:ESI+

Created:11/9/2018 8:49:07 AM

History:Determine m/z[Peak Detect[Centroid,30,Area] ;Correct Base[1.0\%]];Correct Base[5.0\%];Average(MS[1] 1..1) Created by:AccuTOF

Charge number: 1

Element: ${ }^{12} \mathrm{C}: 0$.. 30, ${ }^{1} \mathrm{H}: 0 . .40,{ }^{16} \mathrm{O}: 0$.. 15

Tolerance:3.00(mmu)

Unsaturation Number:0.0 .. 30.0 (Fraction:Both)

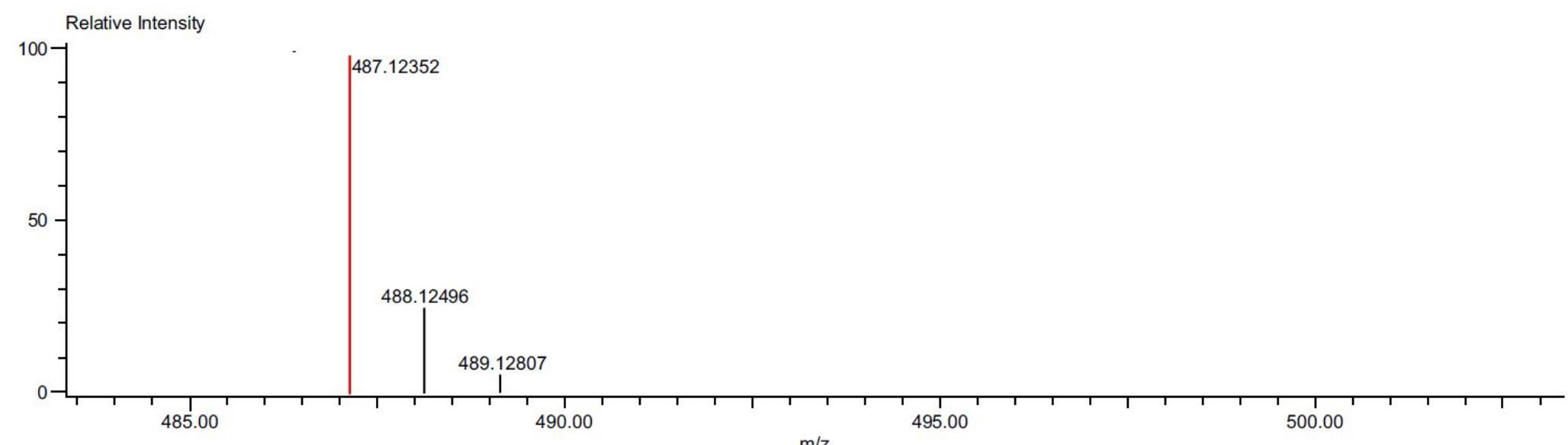

$\mathrm{m} / \mathrm{z}$

\begin{tabular}{|c|c|c|c|c|c|c|}
\hline Mass & Intensity & Calc. Mass & $\begin{array}{c}\text { Mass Difference } \\
(\mathrm{mmu})\end{array}$ & $\begin{array}{c}\text { Mass Difference } \\
(\mathrm{ppm})\end{array}$ & Possible Formula & Unsaturation Number \\
\hline \hline 487.12352 & 111286.38 & 487.12404 & 0.52 & 1.07 & ${ }^{12} \mathrm{C}_{24}{ }^{1} \mathrm{H}_{23}{ }^{16} \mathrm{O}_{11}$ & 13.5 \\
\hline
\end{tabular}

Figure S28. HRESIMS spectrum of $\mathbf{4}$. 


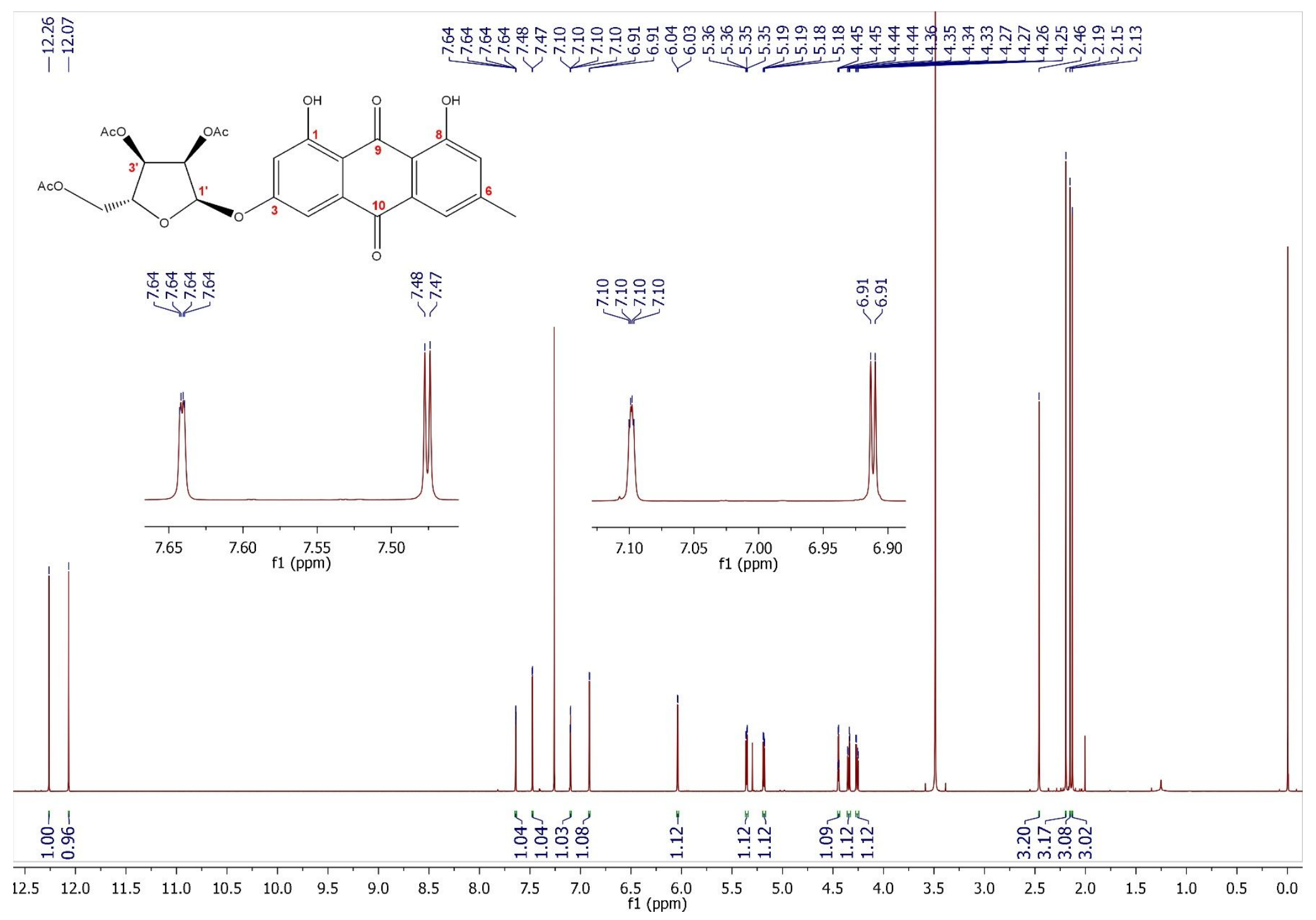

Figure S29. ${ }^{1} \mathrm{H}$ NMR spectrum of 5 in $\mathrm{CDCl}_{3}(700 \mathrm{MHz})$. 


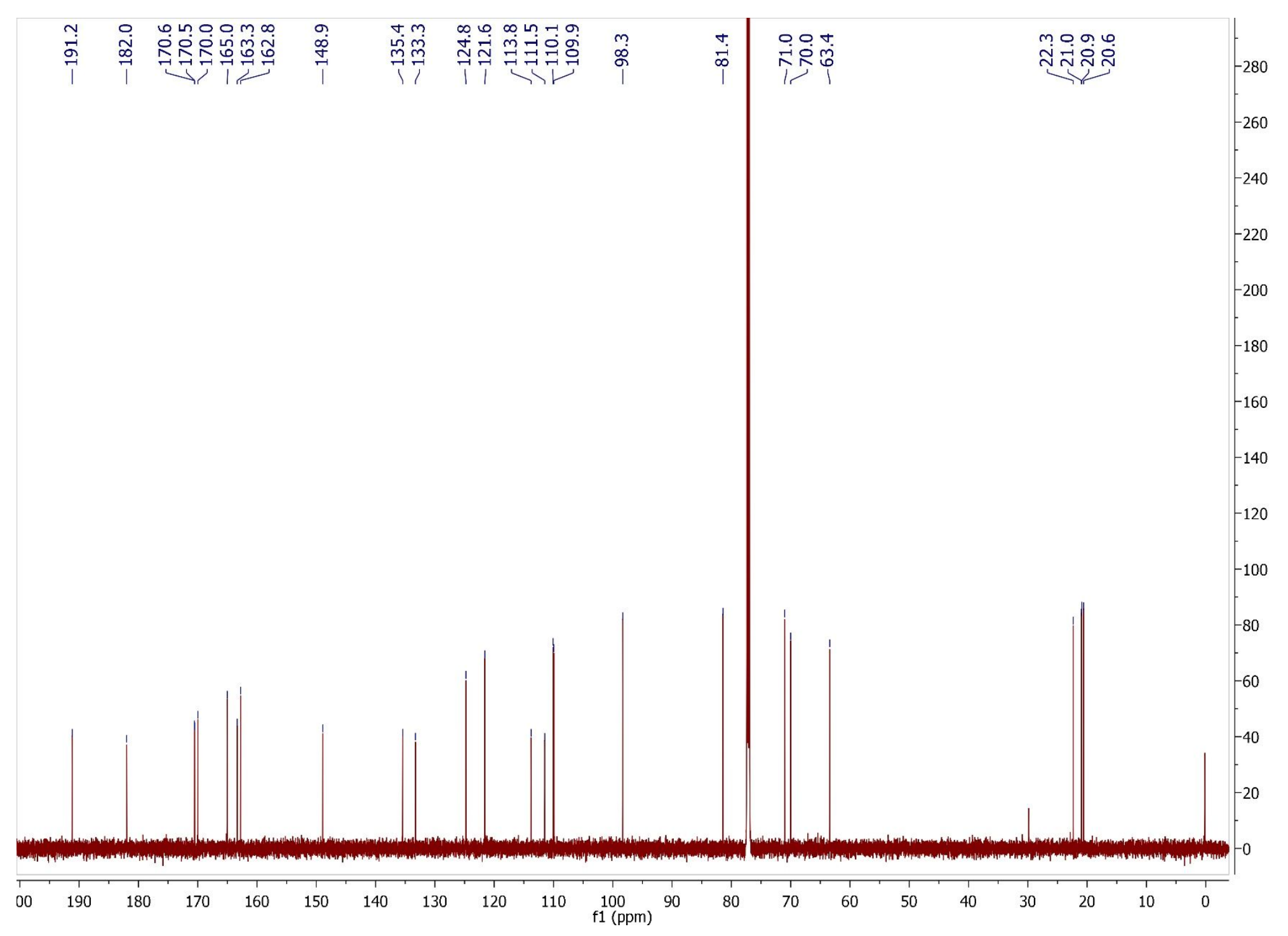

Figure S30. ${ }^{13} \mathrm{C}$ NMR spectrum of 5 in $\mathrm{CDCl}_{3}(175 \mathrm{MHz})$. 


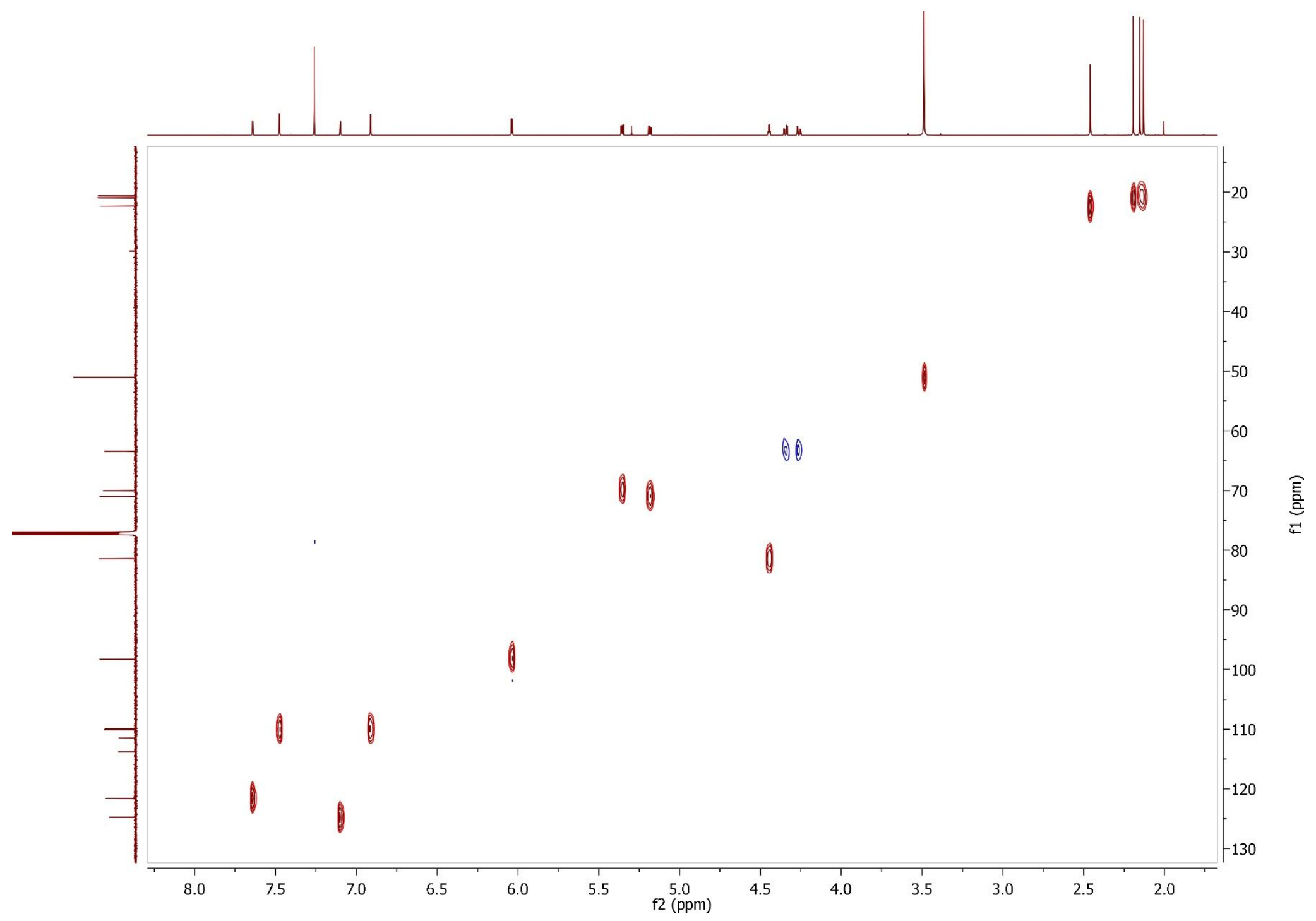

Figure S31. HSQC spectrum of 5 in $\mathrm{CDCl}_{3}$. 


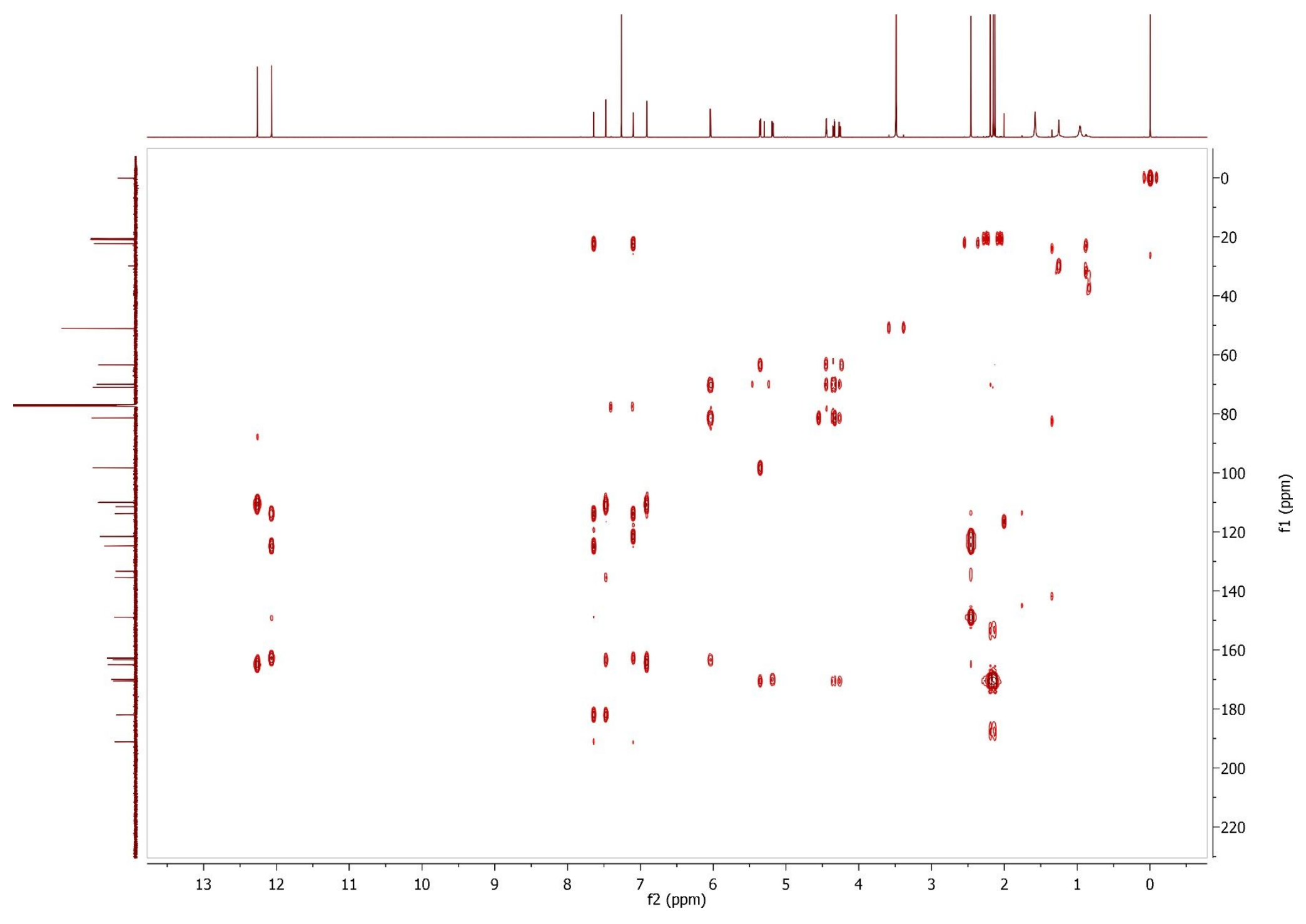

Figure S32. $\mathrm{HMBC}$ spectrum of $\mathbf{5}$ in $\mathrm{CDCl}_{3}$. 


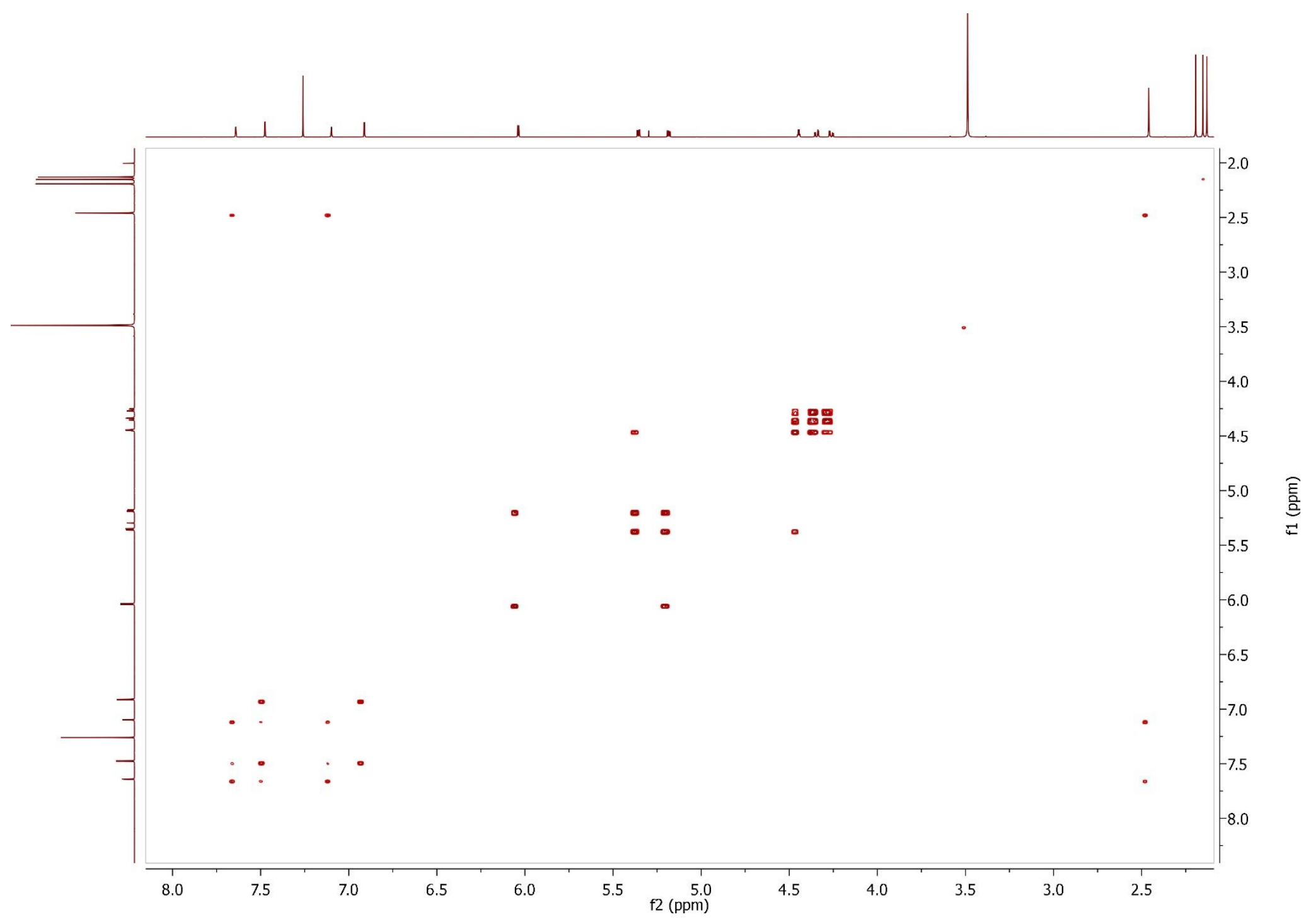

Figure S33. COSY spectrum of 5 in $\mathrm{CDCl}_{3}$. 


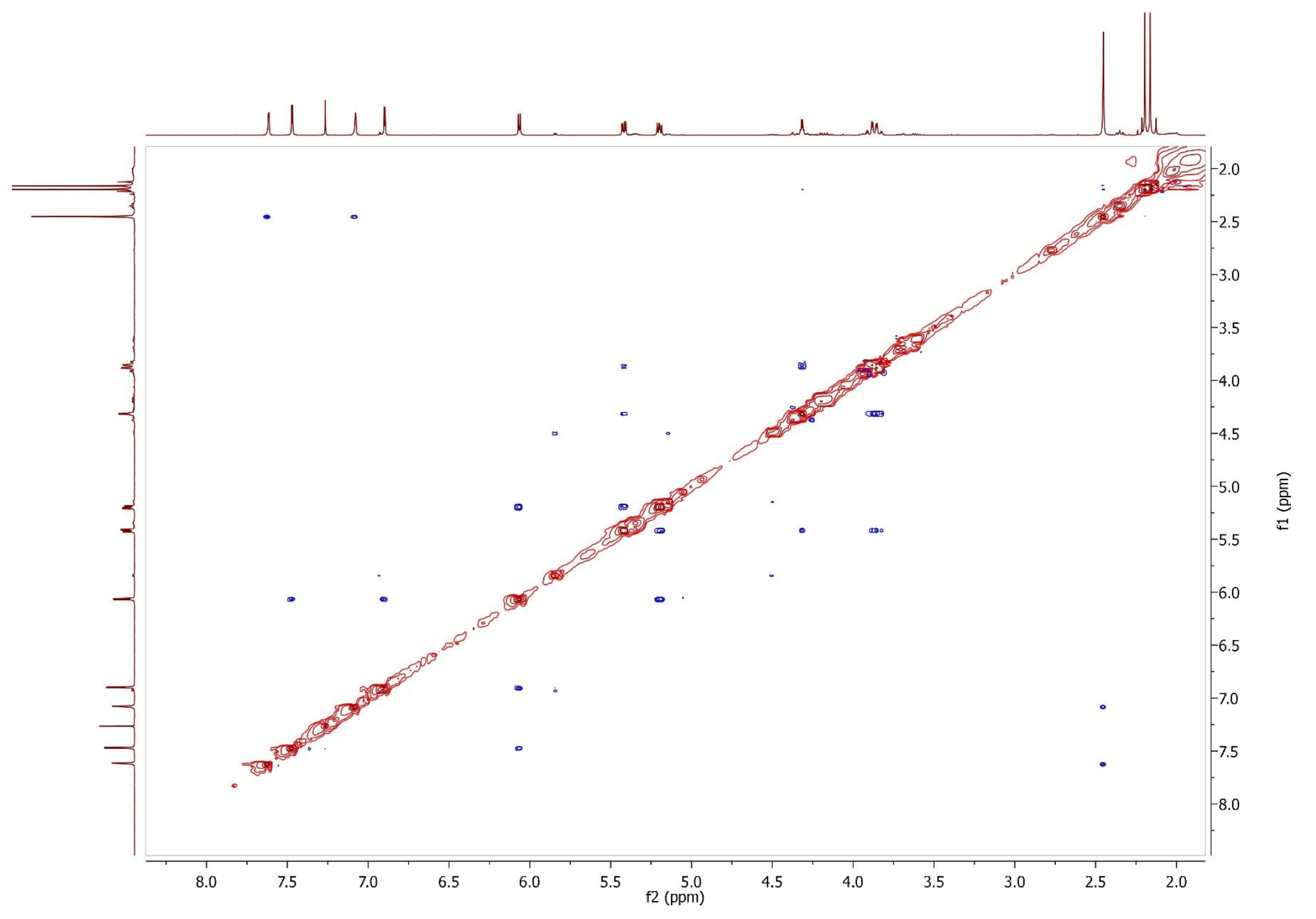

Figure S34. NOESY spectrum of 5 in $\mathrm{CDCl}_{3}$. 


\section{INSTITUTO DE QUIMICA, UNAM}

LABORATORIO DE ESPECTROMETRIA DE MASAS

\section{Data:926 MR15-Ma-H}

Sample Name:Dra Macias Martha / Operador: Carmen Garcia-Javier Perez

Description:

lonization Mode:ESI+

Acquired:5/22/2019 10:50:17 AM

Operator:AccuTOF

Mass Calibration data:Cal Peg 600

History:Determine m/z[Peak Detect[Centroid,30,Area];Correct Base[];Smooth[5]];Correct Base[5.0\%];Average(MS[..

Created:5/31/2019 12:17:55 PM

Charge number: 1

Element: ${ }^{12} \mathrm{C}: 0$.. $30,{ }^{1} \mathrm{H}: 0 . .50,{ }^{16} \mathrm{O}: 0$.. 15

Tolerance:5.00(mmu)

Created by:AccuTOF

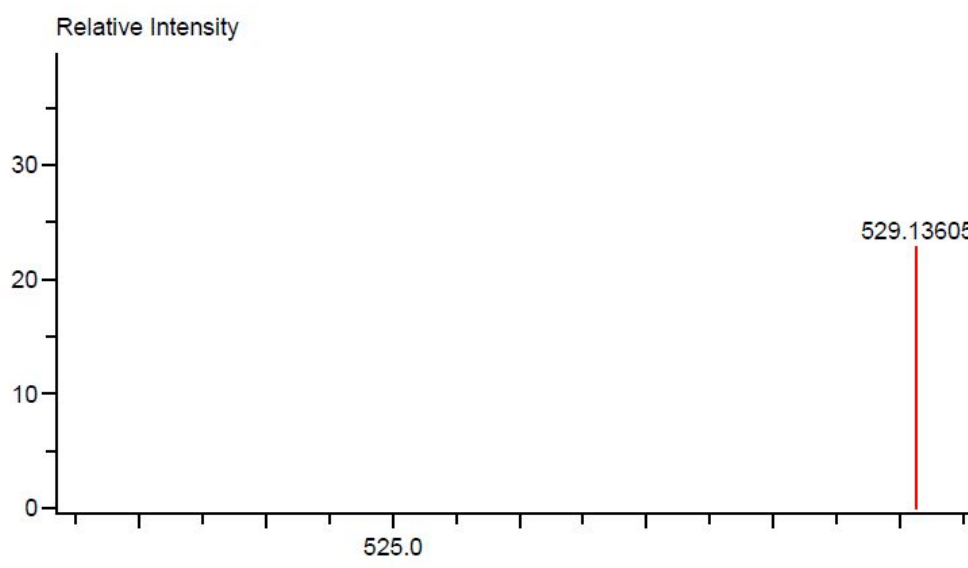

530.13749

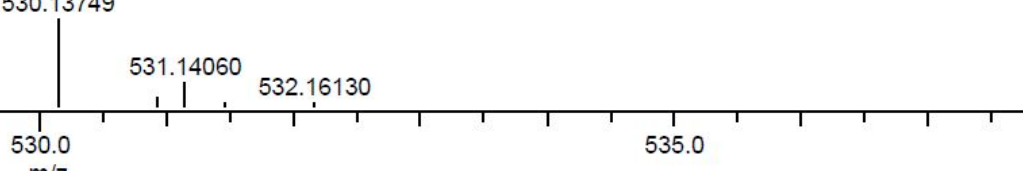

\begin{tabular}{|c|c|c|c|c|c|c|}
\hline Mass & Intensity & Calc. Mass & $\begin{array}{c}\text { Mass Difference } \\
(\mathrm{mmu})\end{array}$ & $\begin{array}{c}\text { Mass Difference } \\
(\mathrm{ppm})\end{array}$ & Possible Formula & Unsaturation Number \\
\hline \hline 529.13605 & 11465.52 & 529.13460 & 1.45 & 2.74 & ${ }^{12} \mathrm{C}_{26}{ }^{1} \mathrm{H}_{25}{ }^{16} \mathrm{O}_{12}$ & 14.5 \\
\hline
\end{tabular}

Figure S35. HRESIMS spectrum of $\mathbf{5}$. 


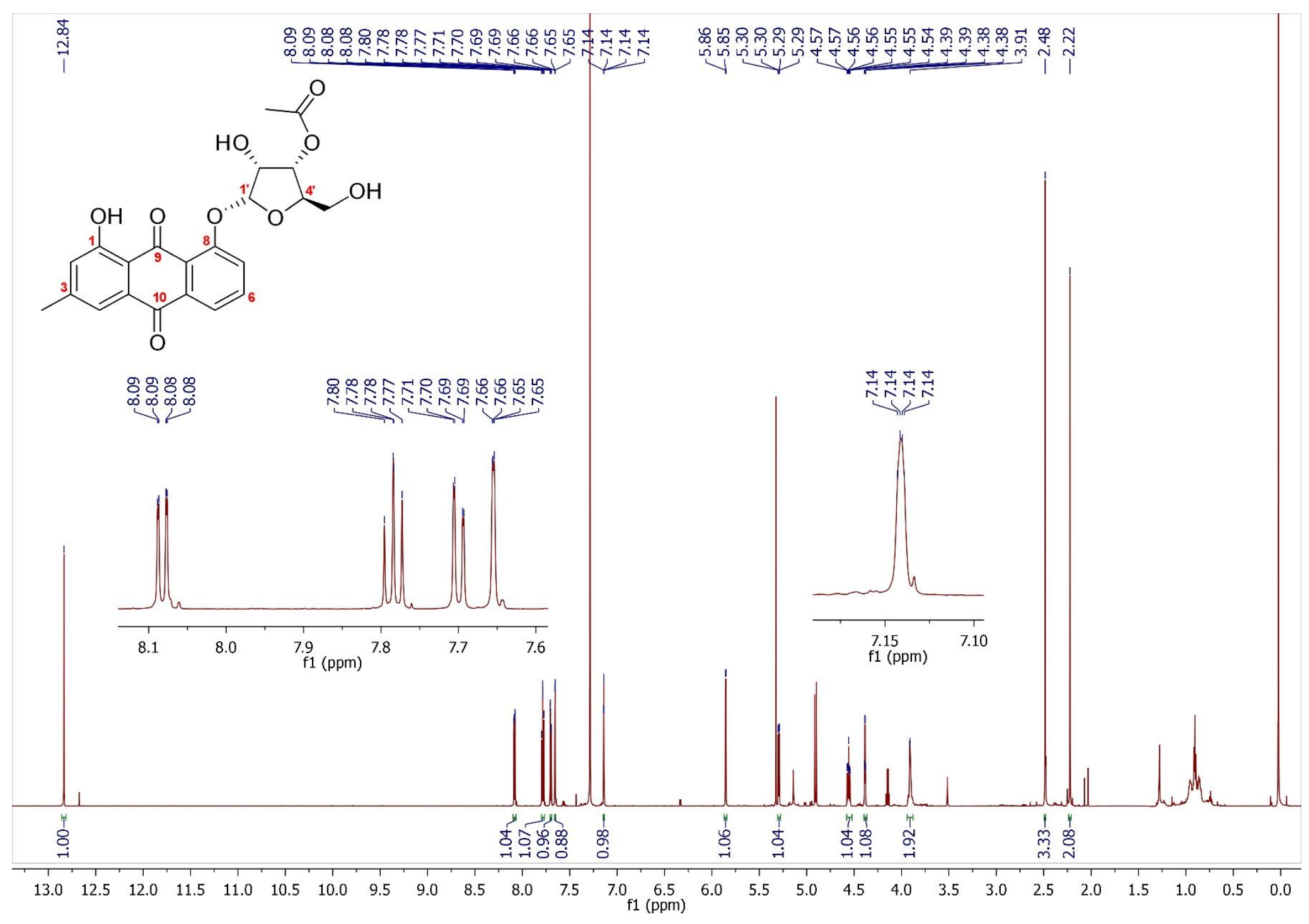

Figure S36. ${ }^{1} \mathrm{H}$ NMR spectrum of 6 in $\mathrm{CDCl}_{3}(700 \mathrm{MHz})$. 


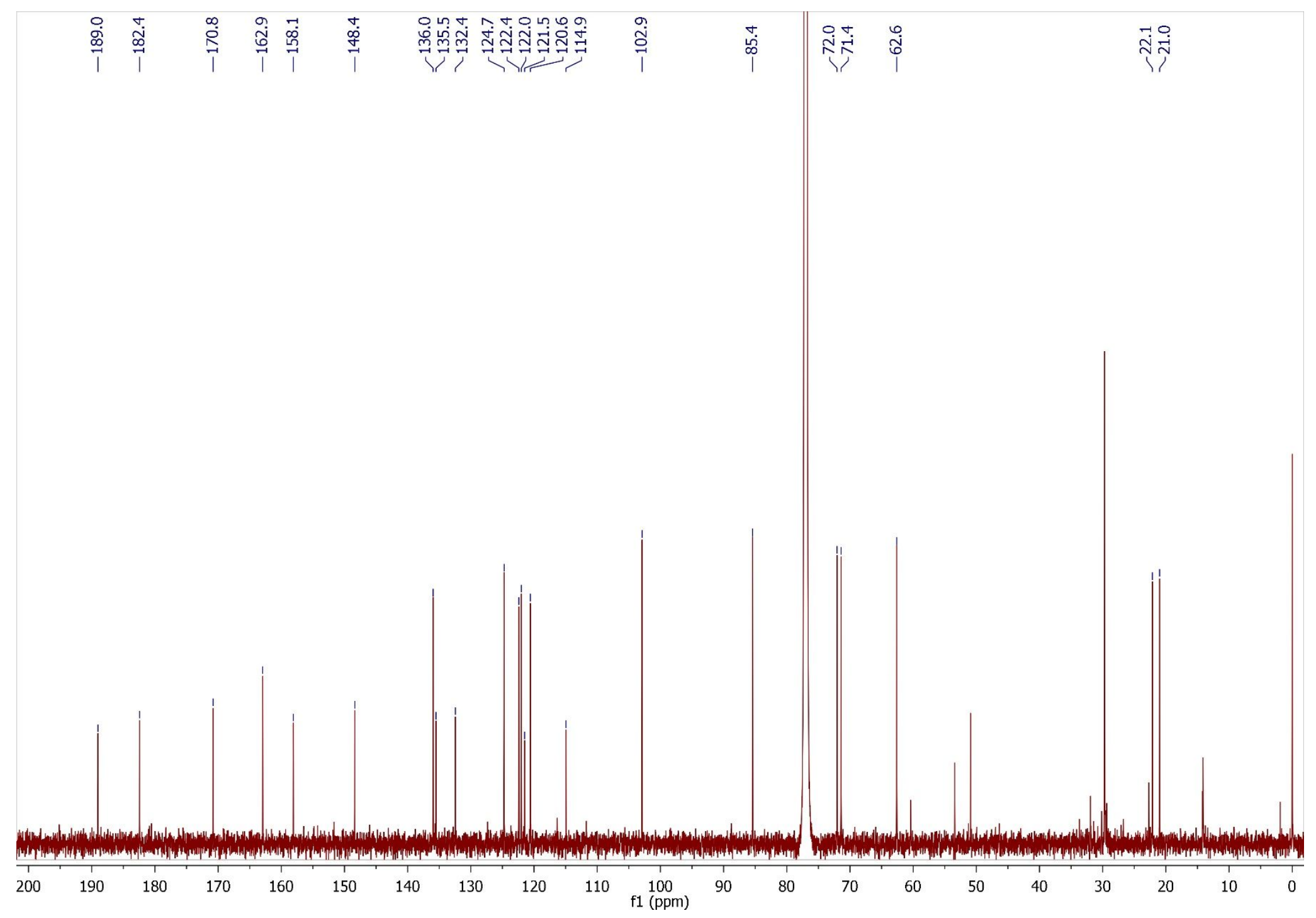

Figure S37. ${ }^{13} \mathrm{C}$ NMR spectrum of 6 in $\mathrm{CDCl}_{3}(175 \mathrm{MHz})$. 


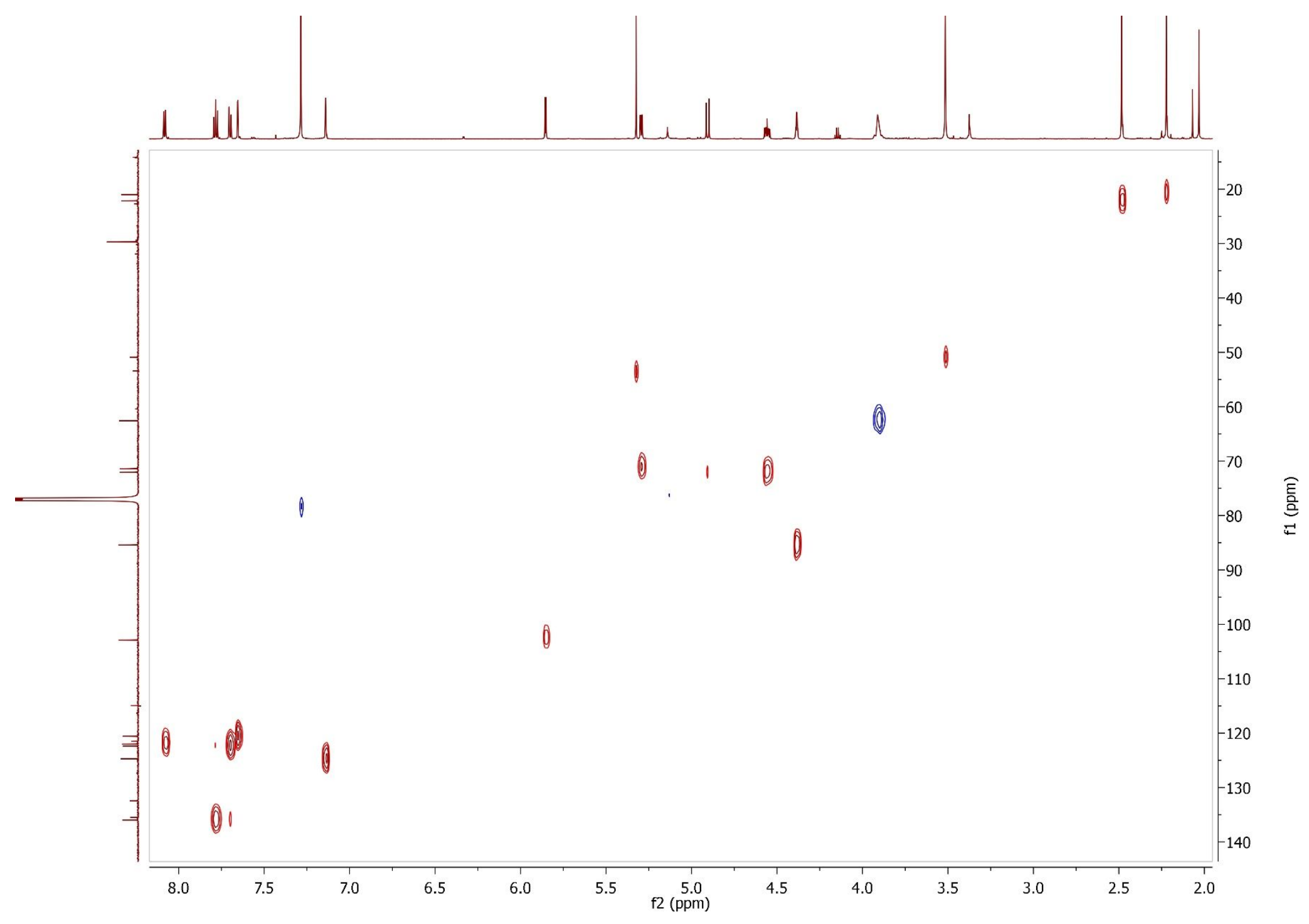

Figure S38. HSQC spectrum of 6 in $\mathrm{CDCl}_{3}$. 


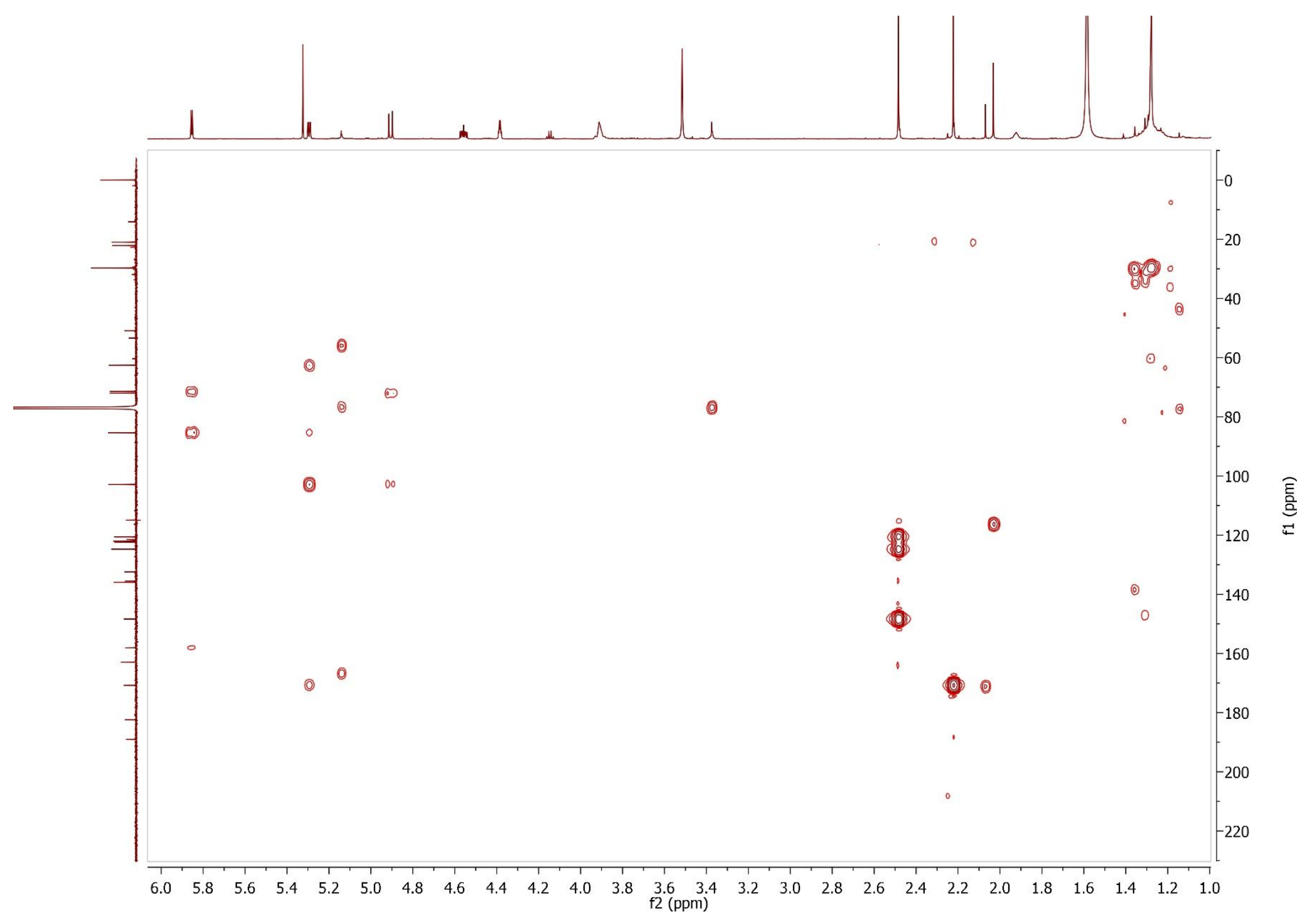

Figure S39. $\mathrm{HMBC}$ spectrum of $\mathbf{6}$ in $\mathrm{CDCl}_{3}$. 


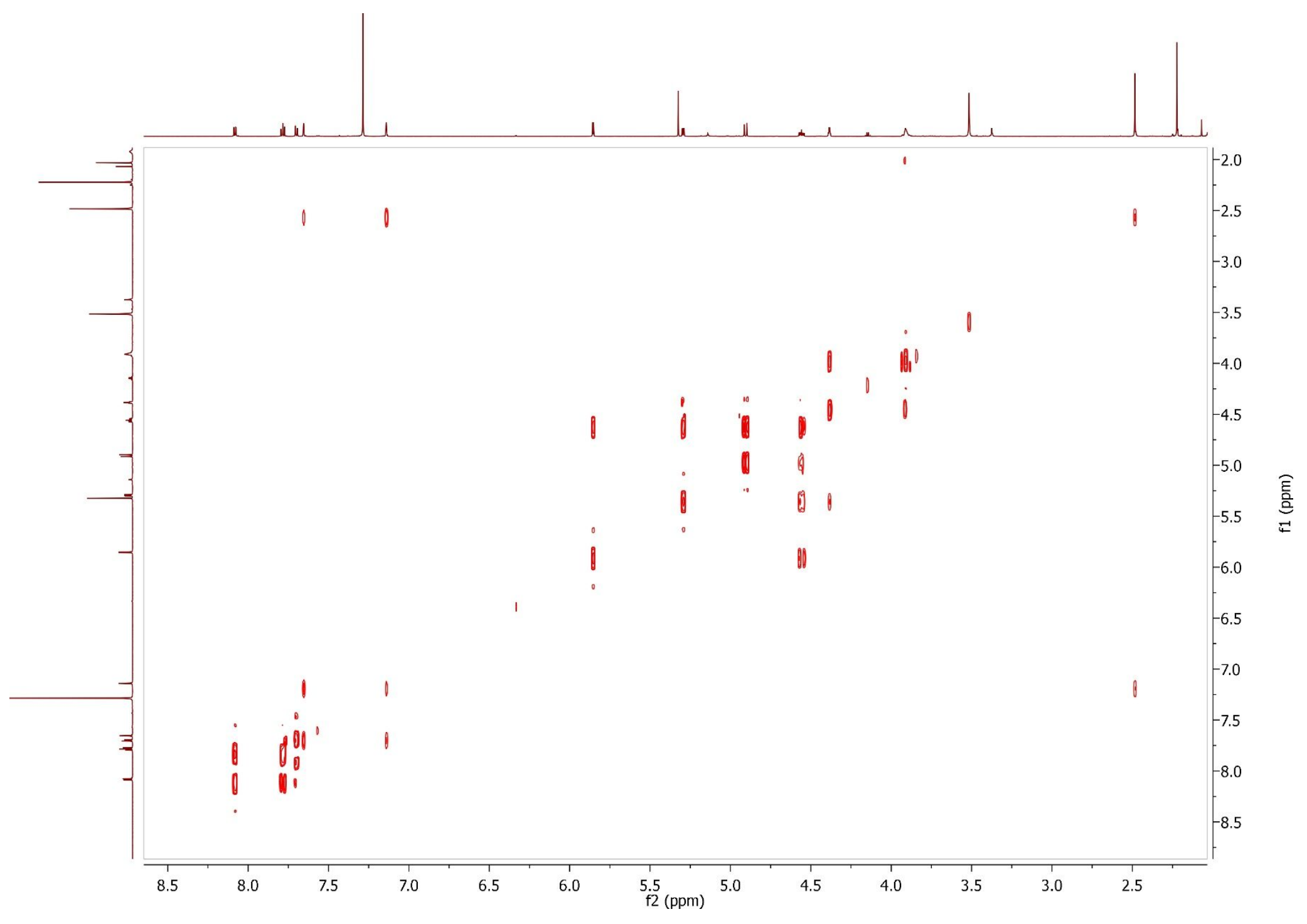

Figure S40. COSY spectrum of 6 in $\mathrm{CDCl}_{3}$. 


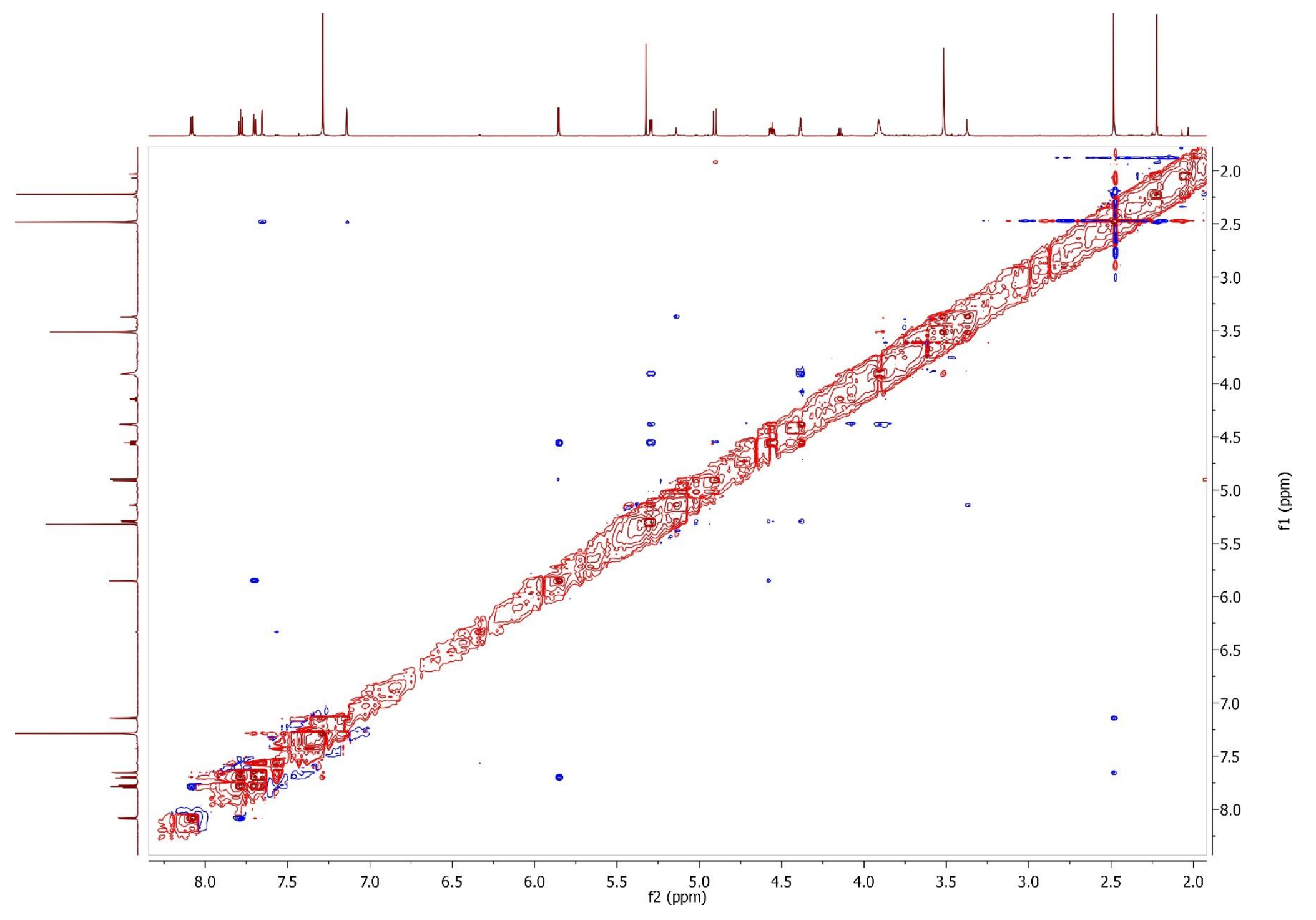

Figure S41. NOESY spectrum of 6 in $\mathrm{CDCl}_{3}$. 
INSTITUTO DE QUIMICA, UNAM

LABORATORIO DE ESPECTROMETRIA DE MASAS

\section{Data:1448 MR15-Ma-U3}

Sample Name:Dra Macias Martha / Operador: Carmen Ga rcia-Javier Perez

Description:

Acquired:8/15/2019 2:45:10 PM

Ionization Mode:ESI+

Operator:AccuTOF

Mass Calibration data:Cal Peg 600

Created:8/26/2019 3:47:29 PM

History:Determine m/z[Peak Detect[Centroid,30,Area] ;Correct Base[;Smooth[5]];Correct Base[5.0\%];Avera ge(MS[...

Created by:AccuTOF

Charge number: 1

Tolerance:3.00(mmu)

Unsaturation Number:0.0 .. 30.0 (Fraction:Both)

Element: ${ }^{12} \mathrm{C}: 0$.. $30,{ }^{1} \mathrm{H}: 0$.. 60, ${ }^{16} \mathrm{O}: 0$.. 15

Relative Intensity

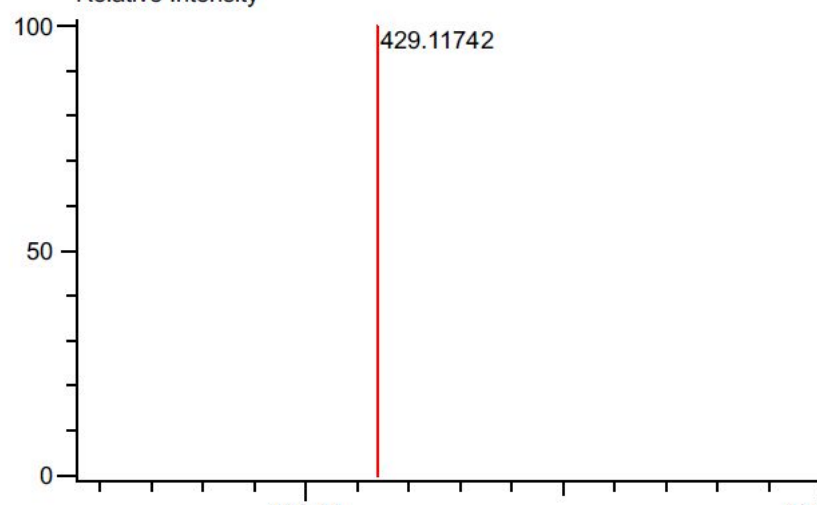

429.00

430.12162

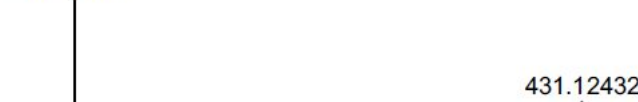

430.00

431.00

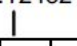

\begin{tabular}{|c|c|c|c|c|c|r|}
\hline Mass & Intensity & Calc. Mass & $\begin{array}{c}\text { Mass Difference } \\
(\mathrm{mmu})\end{array}$ & $\begin{array}{c}\text { Mass Difference } \\
(\mathrm{ppm})\end{array}$ & Possible Formula & Unsaturation Number \\
\hline \hline 429.11742 & 712515.26 & 429.11856 & 1.14 & 2.65 & ${ }^{12} \mathrm{C}_{22}{ }^{1} \mathrm{H}_{21}{ }^{16} \mathrm{O}_{9}$ & 12.5 \\
\hline
\end{tabular}

Figure S42. HRESIMS spectrum of 6. 


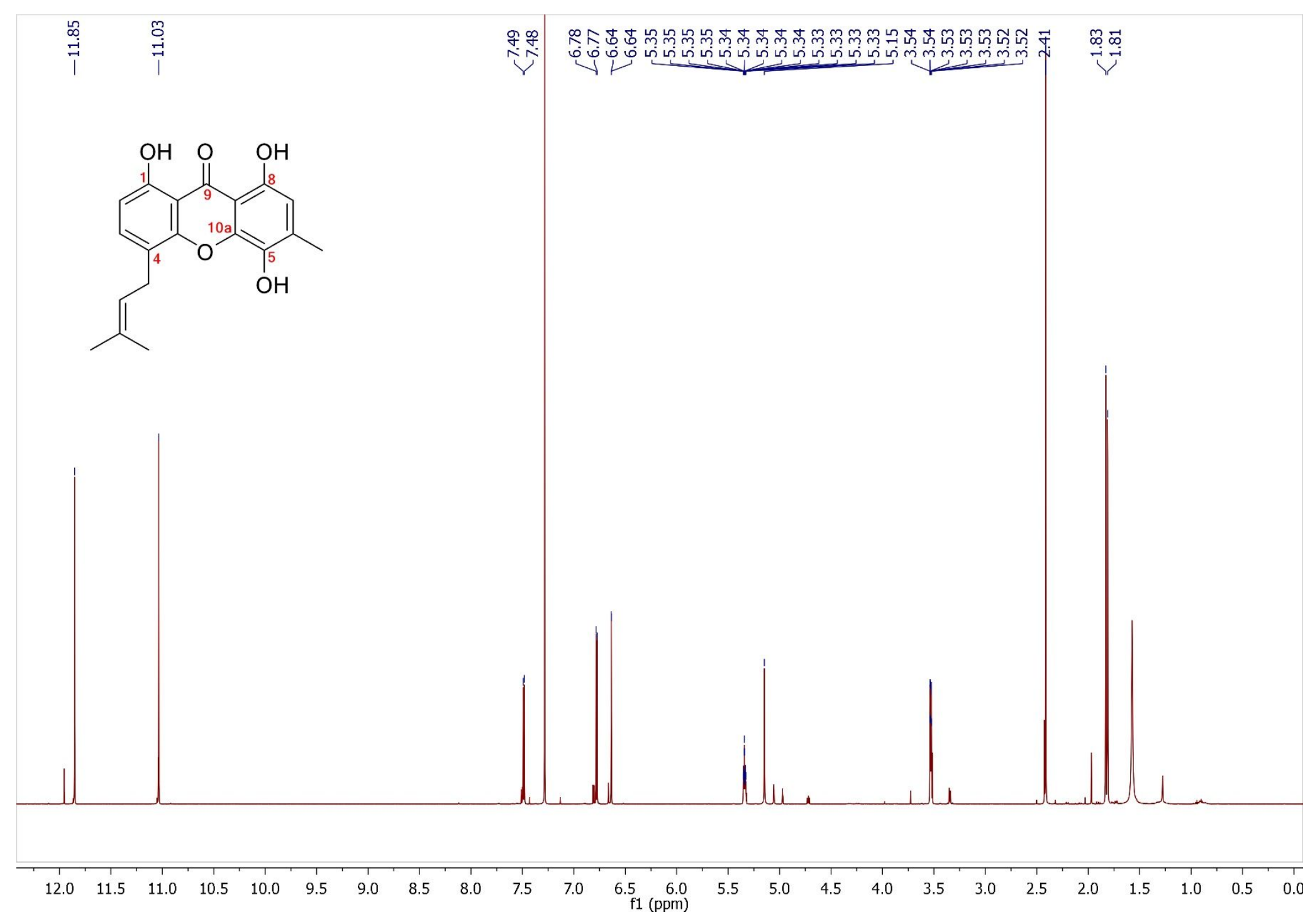

Figure S43. ${ }^{1} \mathrm{H}$ NMR spectrum of 7 in $\mathrm{CDCl}_{3}$. 


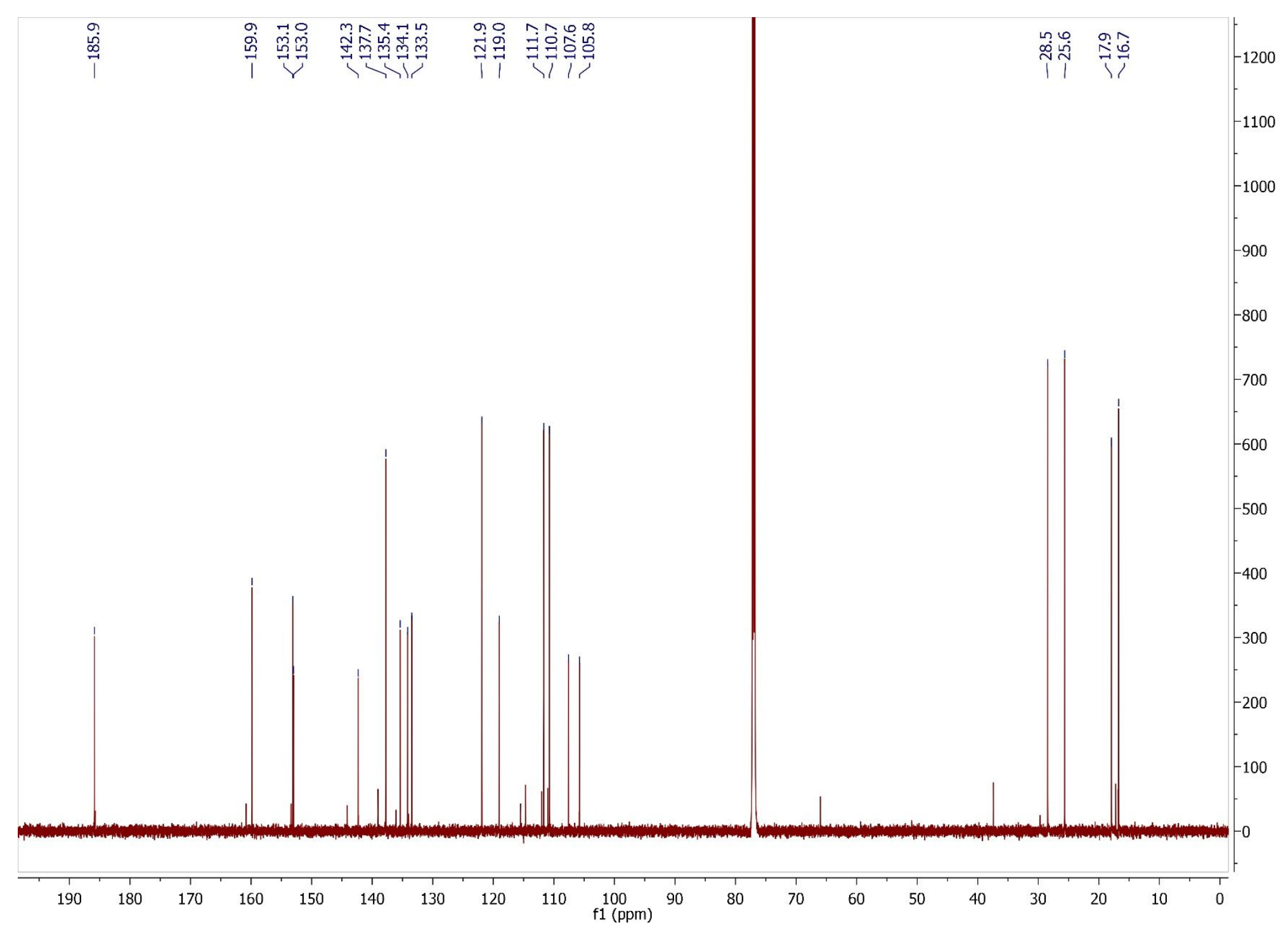

Figure S44. ${ }^{13} \mathrm{C}$ NMR spectrum of 7 in $\mathrm{CDCl}_{3}(175 \mathrm{MHz})$. 


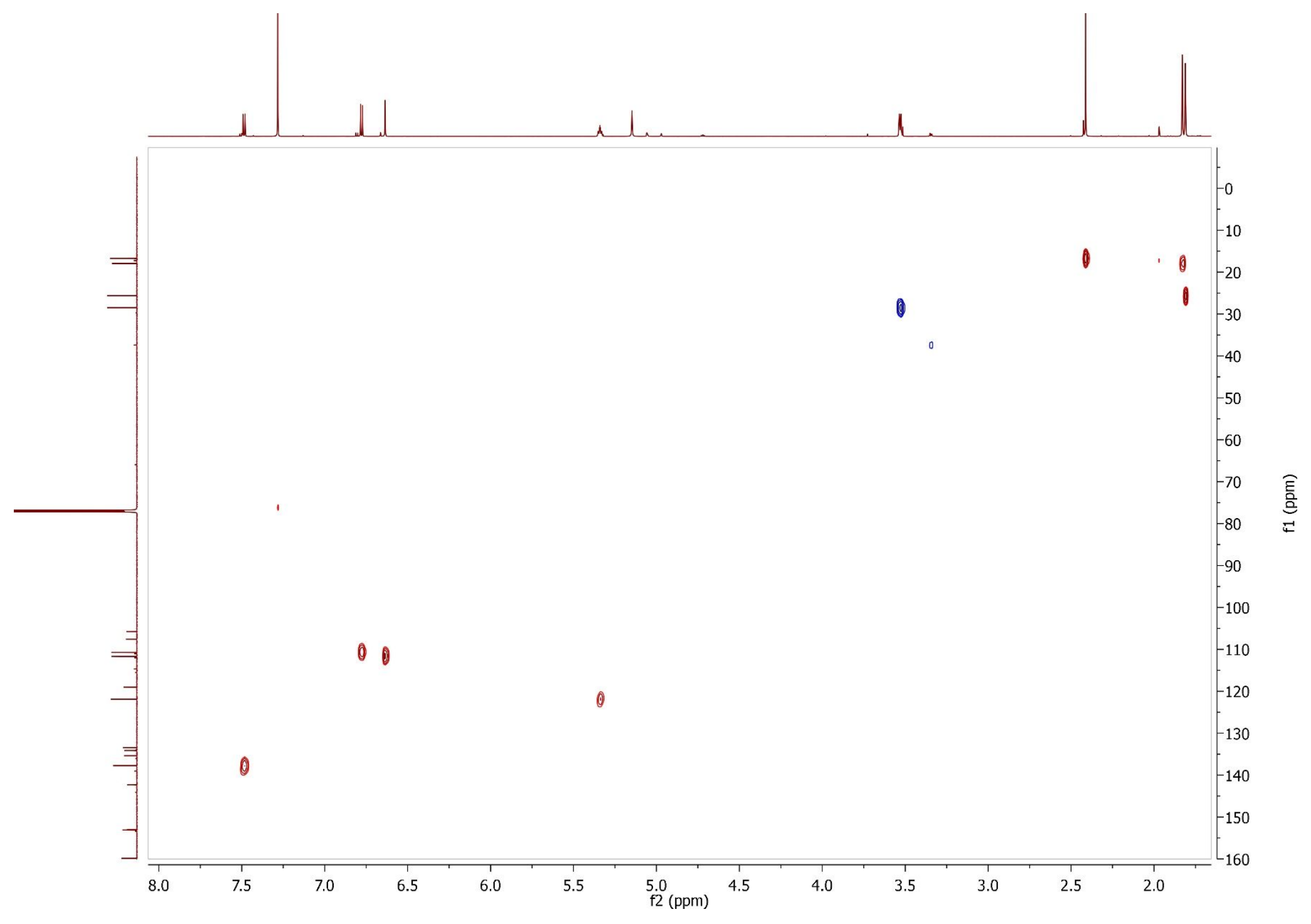

Figure S45. HSQC spectrum of 7 in $\mathrm{CDCl}_{3}$. 


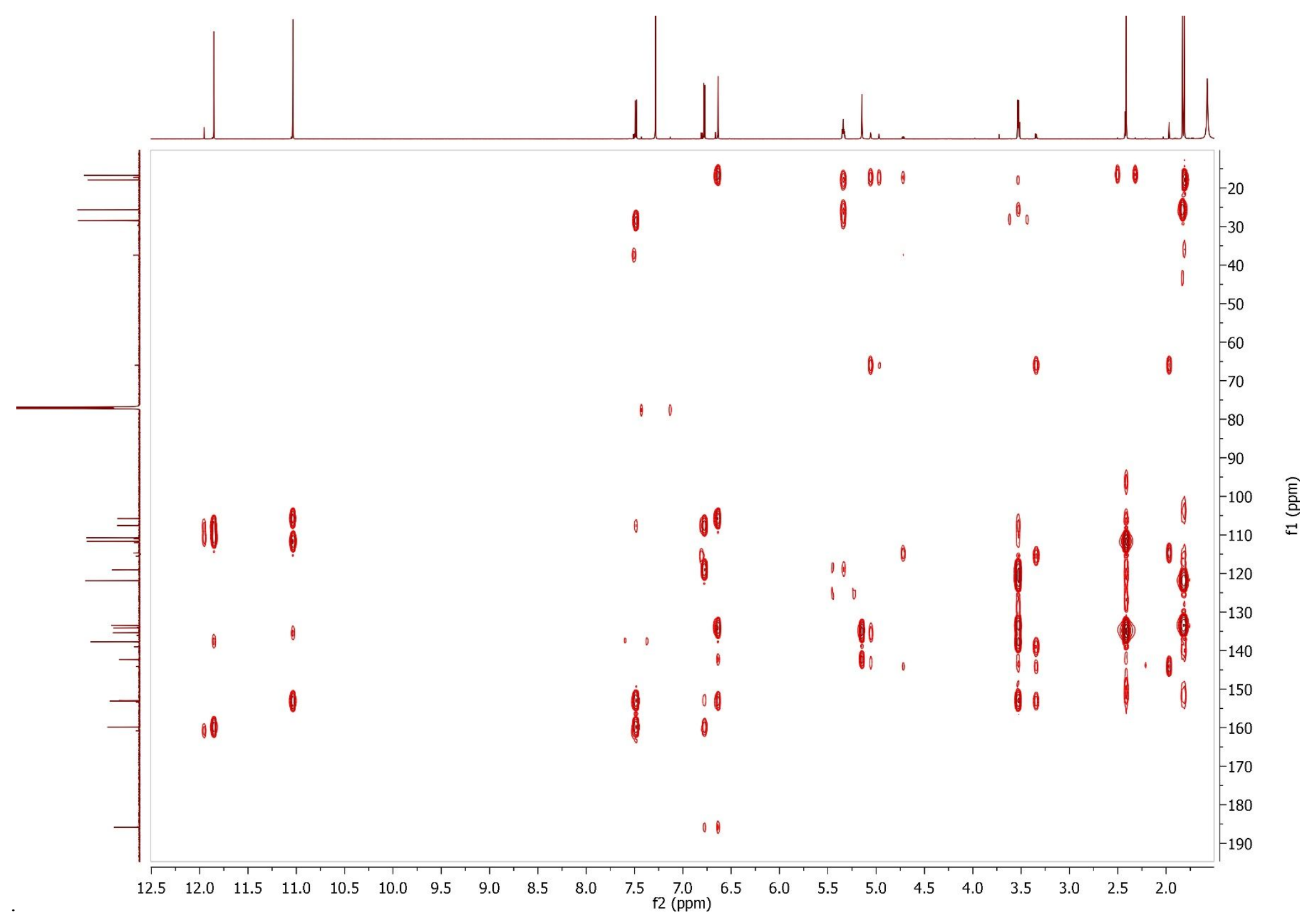

Figure S46. $\mathrm{HMBC}$ spectrum of 7 in $\mathrm{CDCl}_{3}$. 


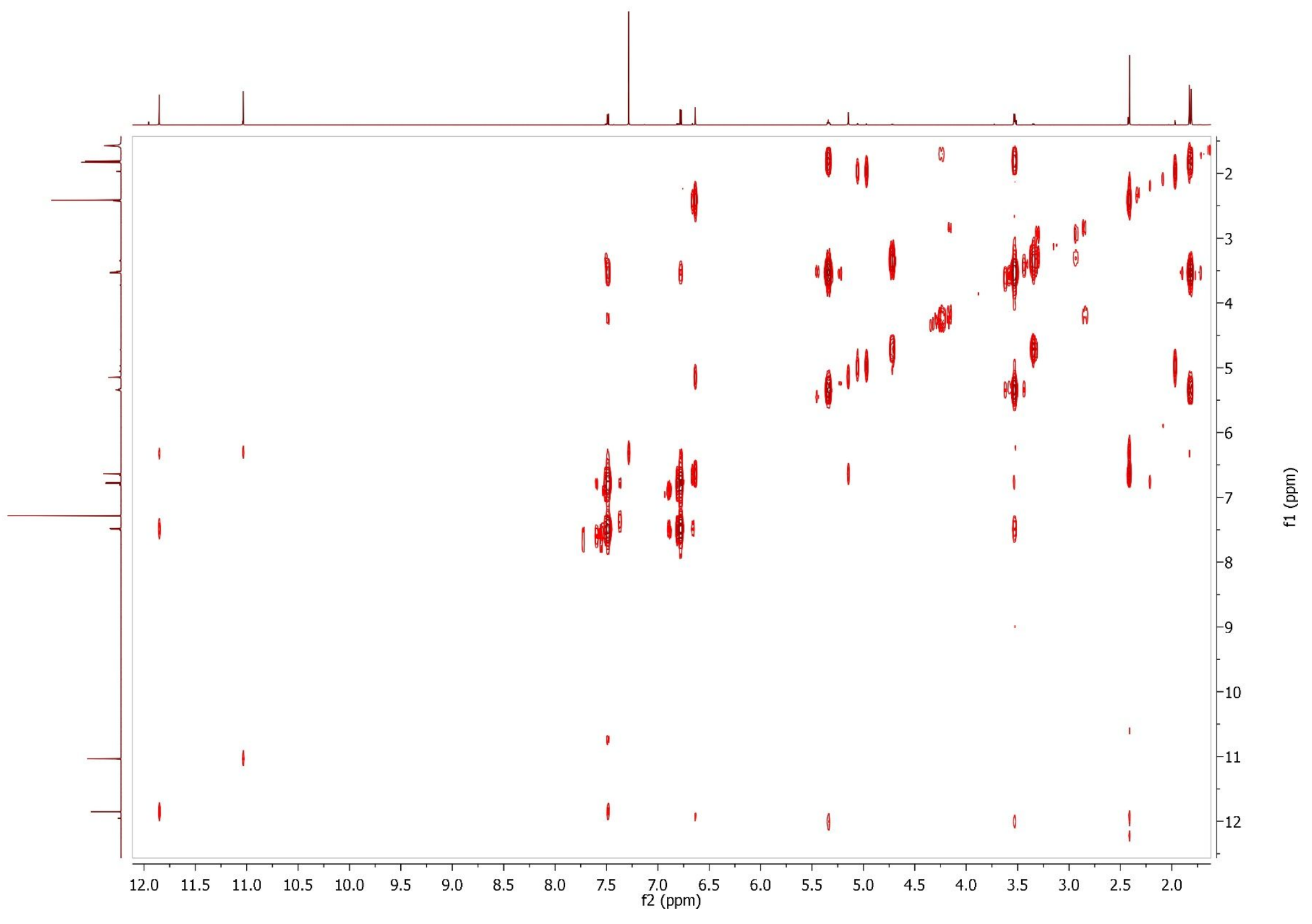

Figure S47. COSY spectrum of 7 in $\mathrm{CDCl}_{3}$. 


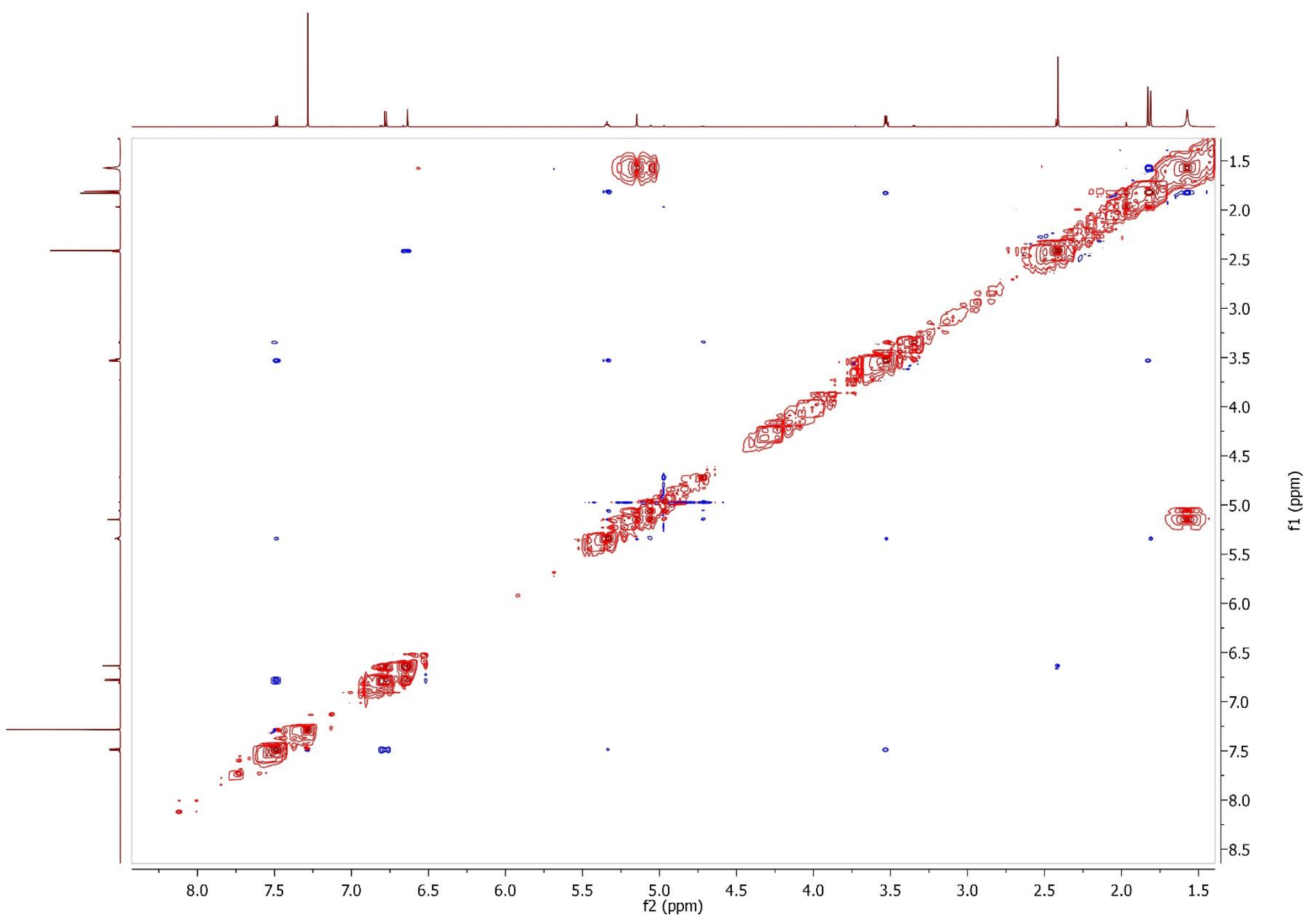

Figure S48. NOESY spectrum of 7 in $\mathrm{CDCl}_{3}$. 
INSTITUTO DE QUIMICA, UNAM

LABORATORIO DE ESPECTROMETRIA DE MASAS

\section{Data:2538 MR15-Ma-R1}

Sample Name:Dra Macias Martha / Operador: Carmen G arcia-Javier Perez

Description:

lonization Mode:ESI+

10:58:24 AM

Operator:AccuTOF

Mass Calibration data:Cal Peg 600

History:Determine m/z[Peak Detect[Centroid,30,Area] ;Correct Base];Smooth[5]];Correct Base[5.0\%];Avera ge(MS[...

Created:5/31/2019 13:12:32 PM

Charge number: 1

Element: ${ }^{12} \mathrm{C}: 0$.. 30, ${ }^{1} \mathrm{H}: 0$.. 50, ${ }^{16} \mathrm{O}: 0$.. 15

Tolerance:5.00(mmu)

Unsaturation Number:0.0 .. 50.0 (Fraction:Both)

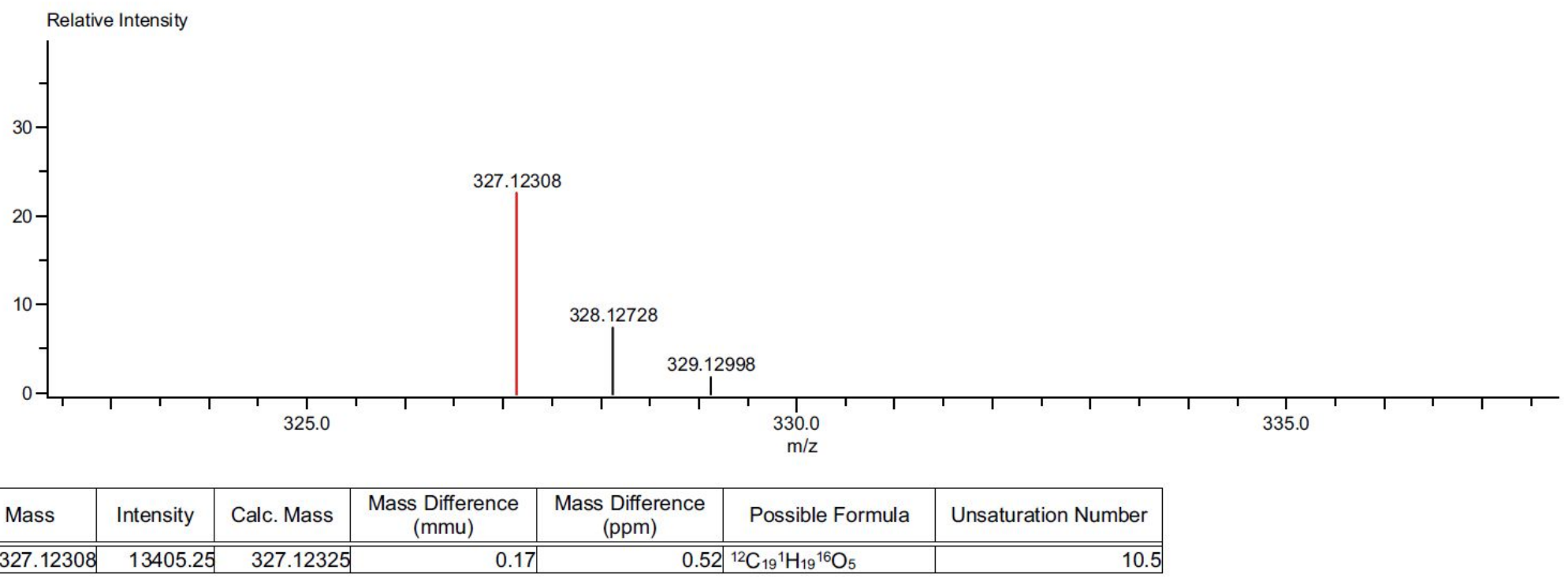

Figure S49. HRESIMS spectrum of 7. 


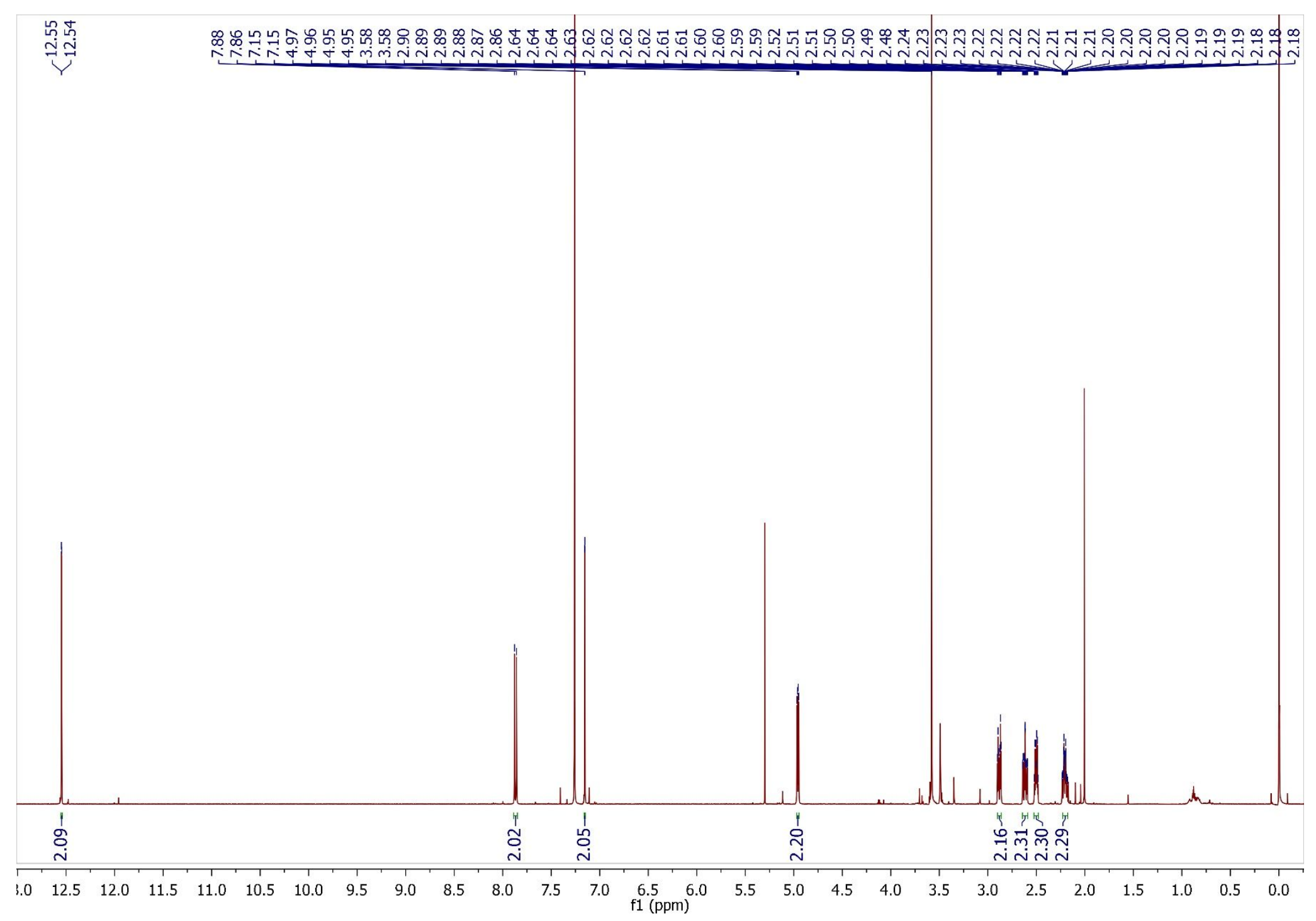

Figure S50. ${ }^{1} \mathrm{H}$ NMR spectrum of 8 in $\mathrm{CDCl}_{3}(700 \mathrm{MHz})$. 


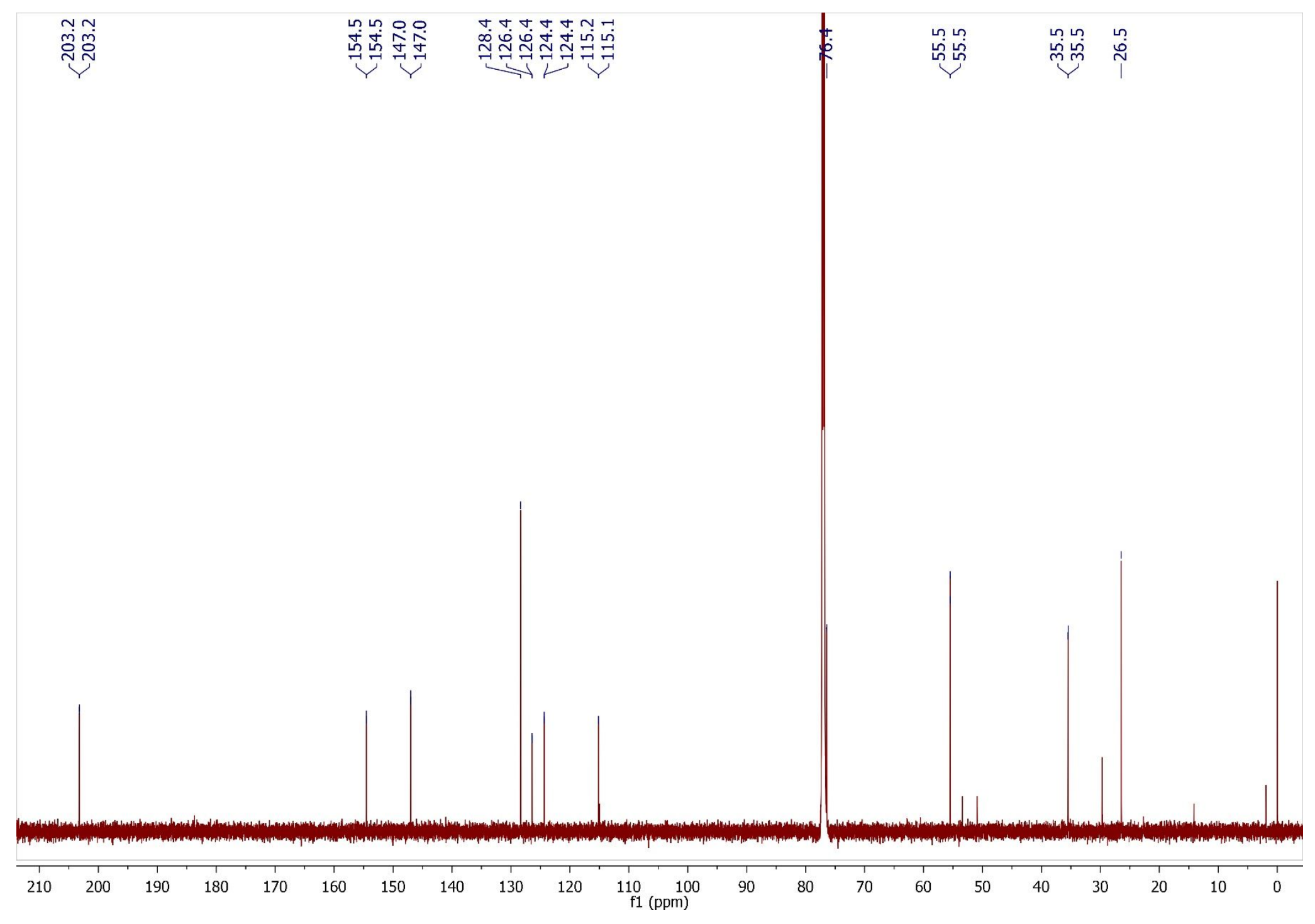

Figure S51. ${ }^{13} \mathrm{C}$ NMR spectrum of 8 in $\mathrm{CDCl}_{3}(175 \mathrm{MHz})$. 


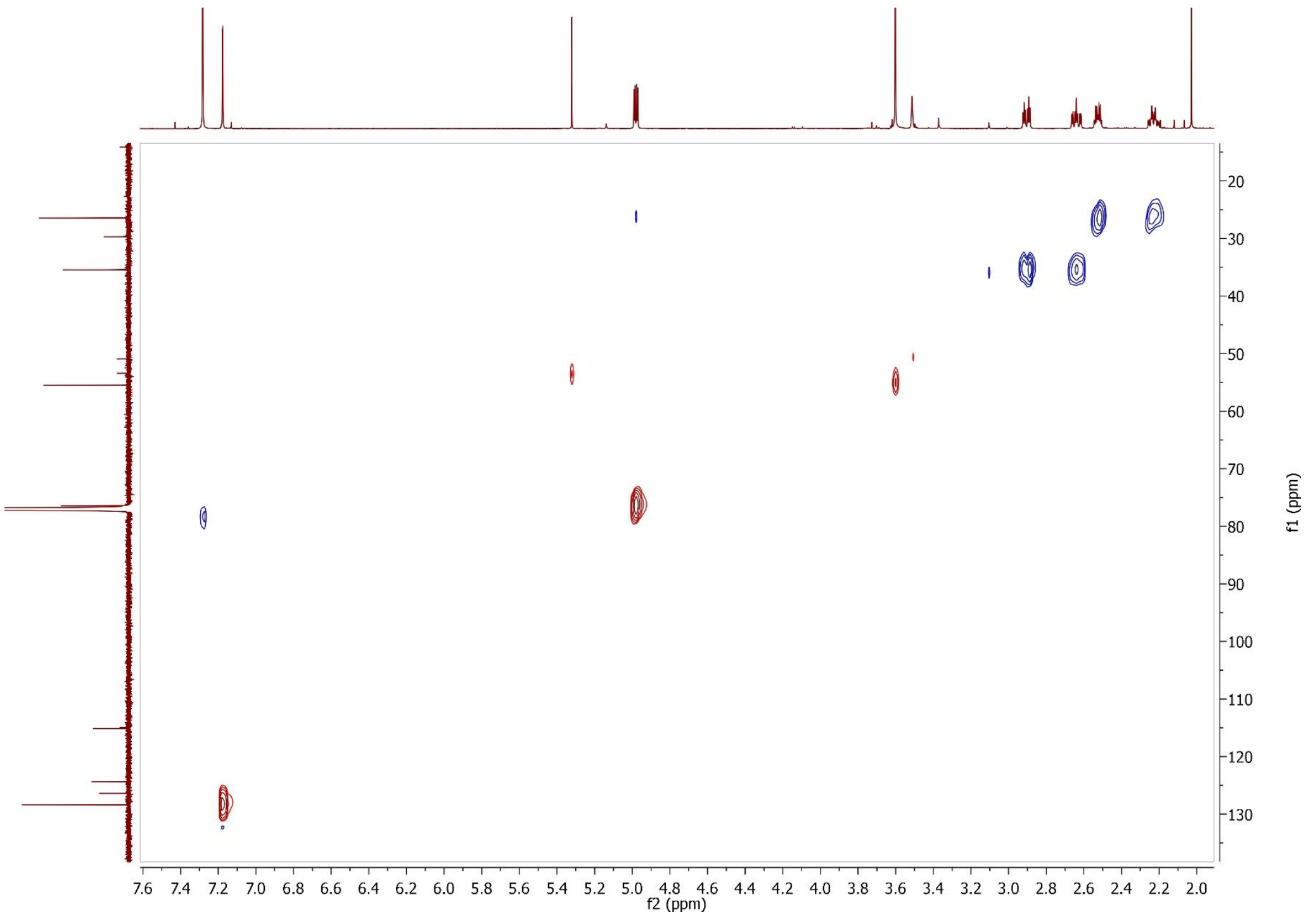

Figure S52. HSQC spectrum of 8 in $\mathrm{CDCl}_{3}$.

55 


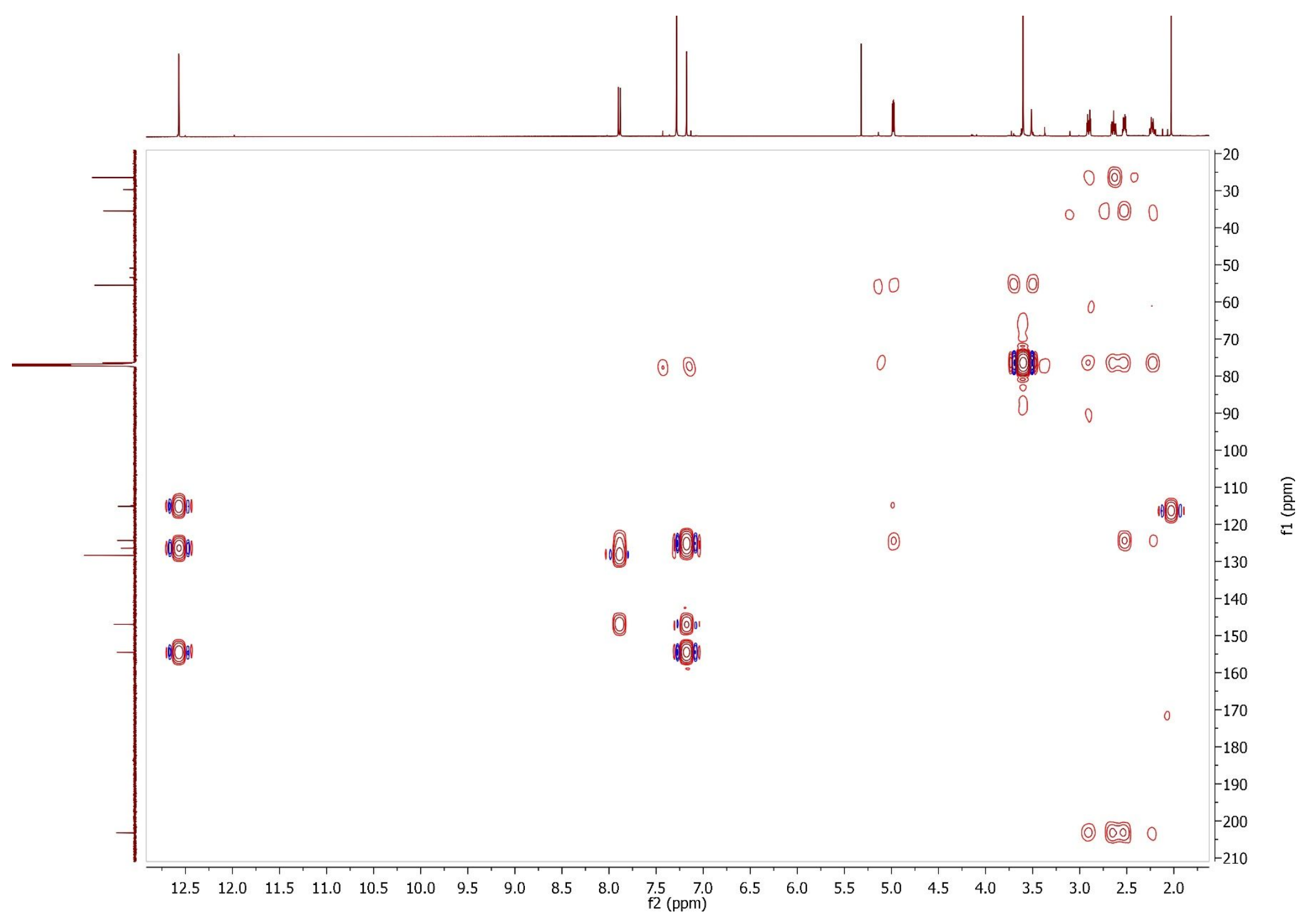

Figure S53. $\mathrm{HMBC}$ spectrum of $\mathbf{8}$ in $\mathrm{CDCl}_{3}$. 


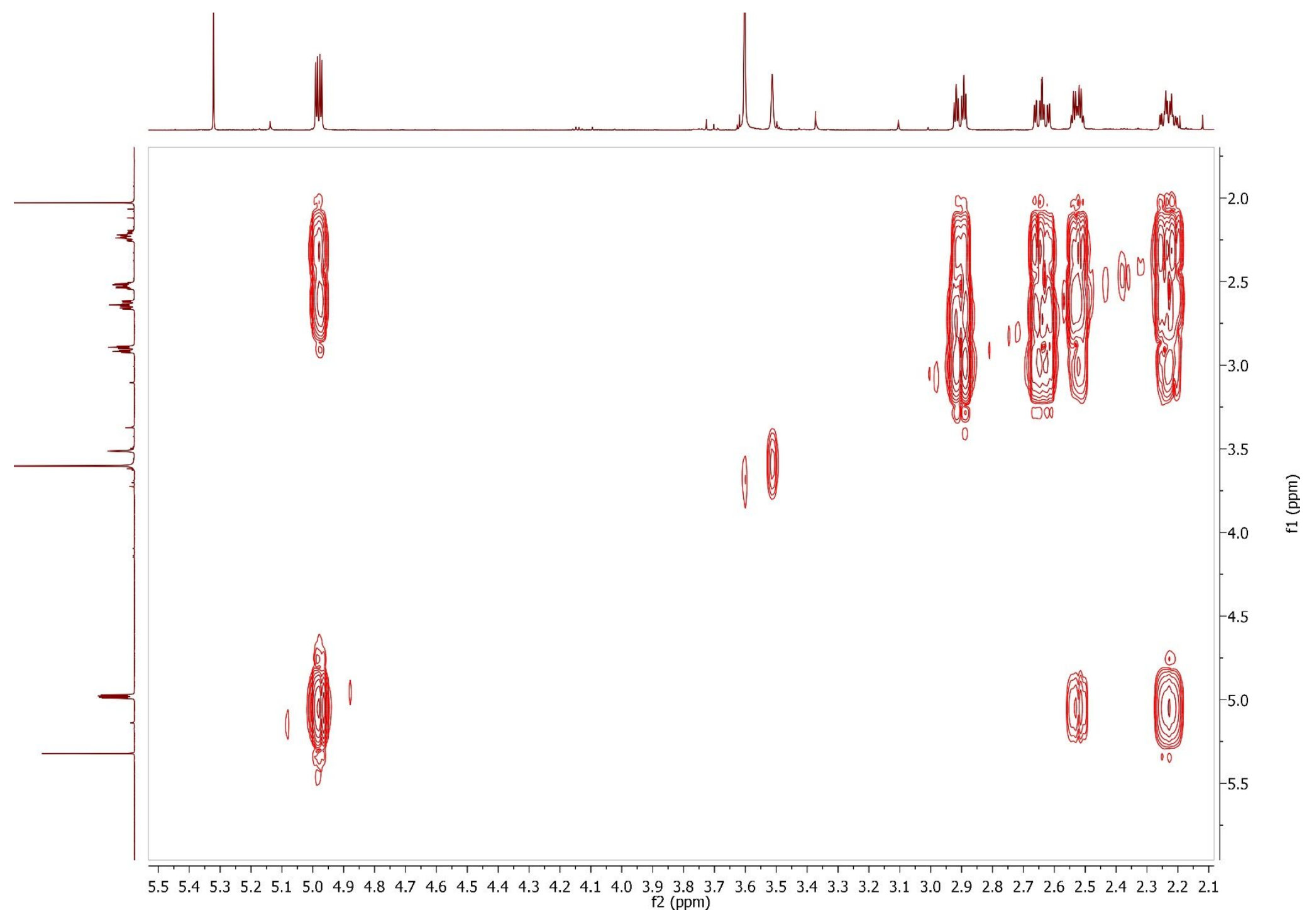

Figure S54. COSY spectrum of 8 in $\mathrm{CDCl}_{3}$. 


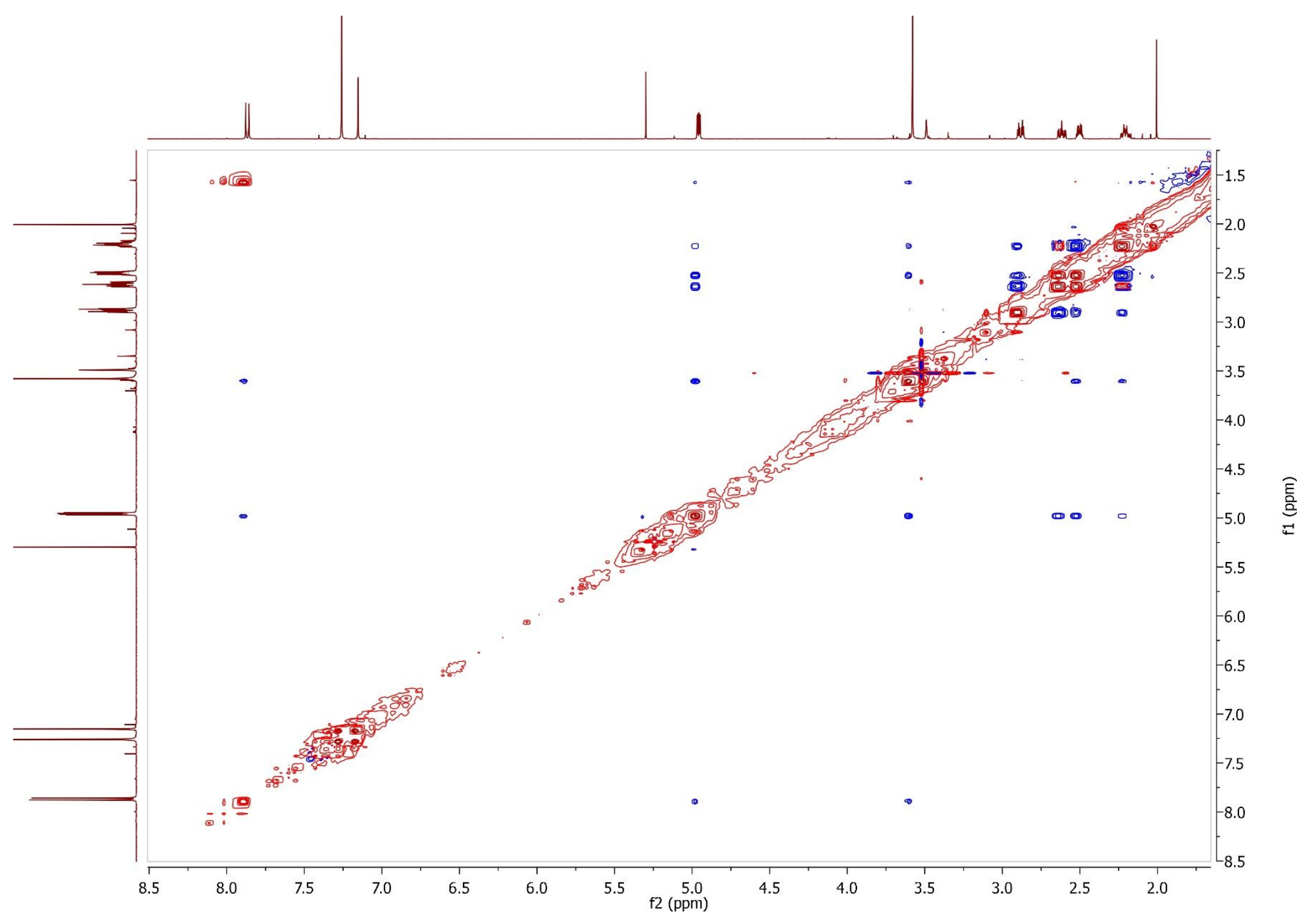

Figure S55. NOESY spectrum of $\mathbf{8}$ in $\mathrm{CDCl}_{3}$. 
INSTITUTO DE QUIMICA, UNAM

LABORATORIO DE ESPECTROMETRIA DE MASAS

\section{Data:1324 MR15-Ma-T22}

Sample Name:Dra Macias Martha / Operador: Carmen Garcia-Javier Perez

Description:

lonization Mode:ESI+

Acquired:8/15/2019 3:11:10 PM

Operator:AccuTOF

Mass Calibration data:Cal Peg 600

Created:8/26/2019 3:13:09 PM

History:Determine m/z[Peak Detect[Centroid,30,Area];Correct Base[];Smooth[5]];Correct Base[5.0\%];Average(MS[... Created by:AccuTOF

Charge number: 1

Element: ${ }^{12} \mathrm{C}: 0 . .30,{ }^{1} \mathrm{H}: 0$.. 60, ${ }^{16} \mathrm{O}: 0 . .8$

Tolerance: $3.00(\mathrm{mmu})$

Unsaturation Number:0.0 .. 40.0 (Fraction:Both)

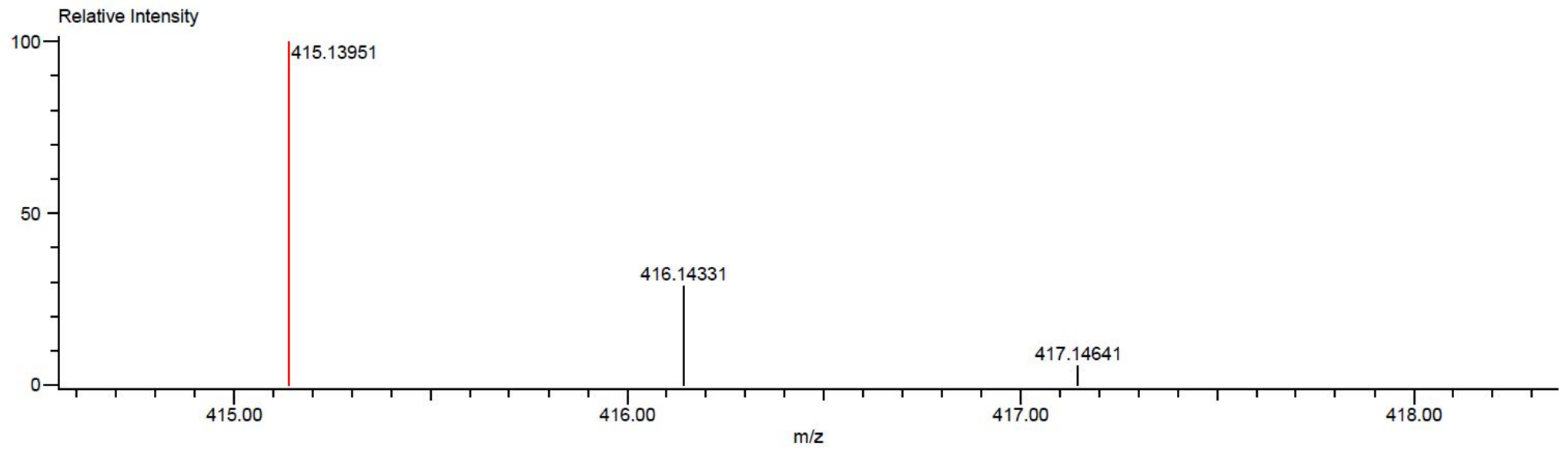

\begin{tabular}{|c|c|r|r|r|r|r|}
\hline Mass & Intensity & Calc. Mass & $\begin{array}{c}\text { Mass Difference } \\
(\mathrm{mmu})\end{array}$ & $\begin{array}{c}\text { Mass Difference } \\
(\mathrm{ppm})\end{array}$ & Possible Formula & Unsaturation Number \\
\hline \hline 415.13951 & 622015.54 & 415.13929 & 0.22 & 0.52 & ${ }^{12} \mathrm{C}_{22}{ }^{1} \mathrm{H}_{23}{ }^{16} \mathrm{O}_{8}$ & 11.5 \\
\hline
\end{tabular}

Figure S56. HRESIMS spectrum of $\mathbf{8}$. 


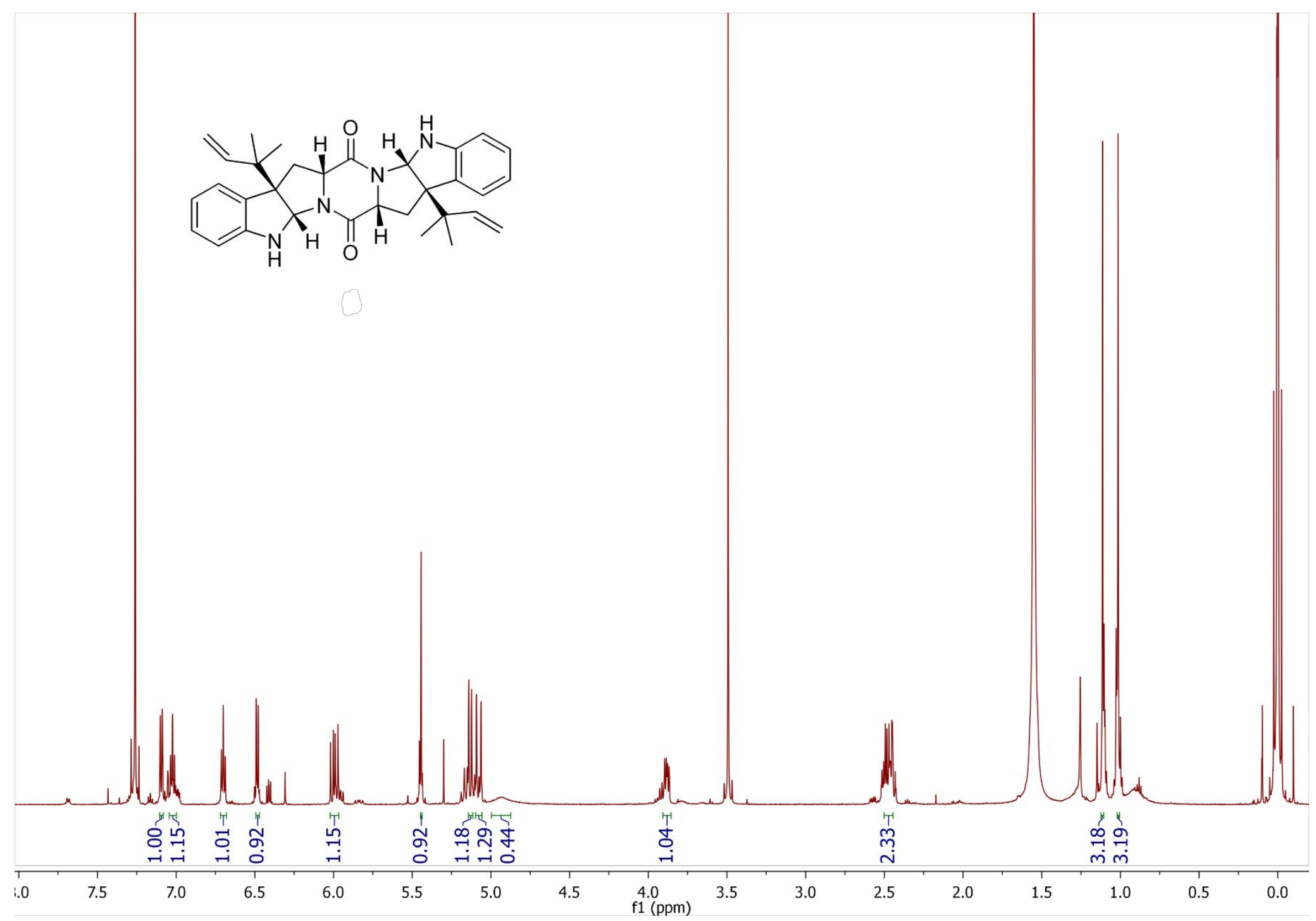

Figure S57. ${ }^{1} \mathrm{H}$ NMR spectrum of 9 in $\mathrm{CD}_{3} \mathrm{OD}(700 \mathrm{MHz})$. 


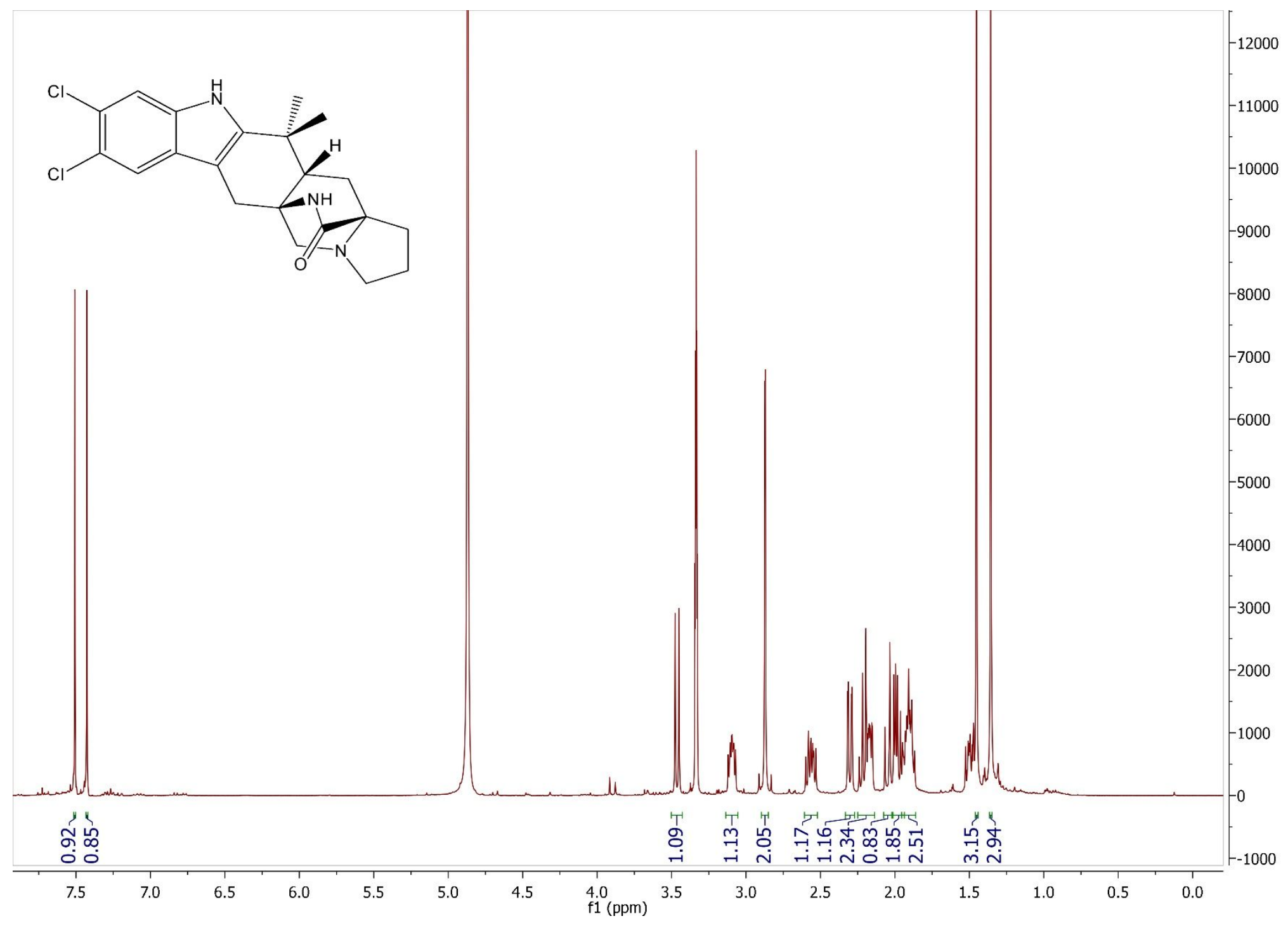

Figure S58. ${ }^{1} \mathrm{H}$ NMR spectrum of 10 in $\mathrm{CD}_{3} \mathrm{OD}(700 \mathrm{MHz})$. 


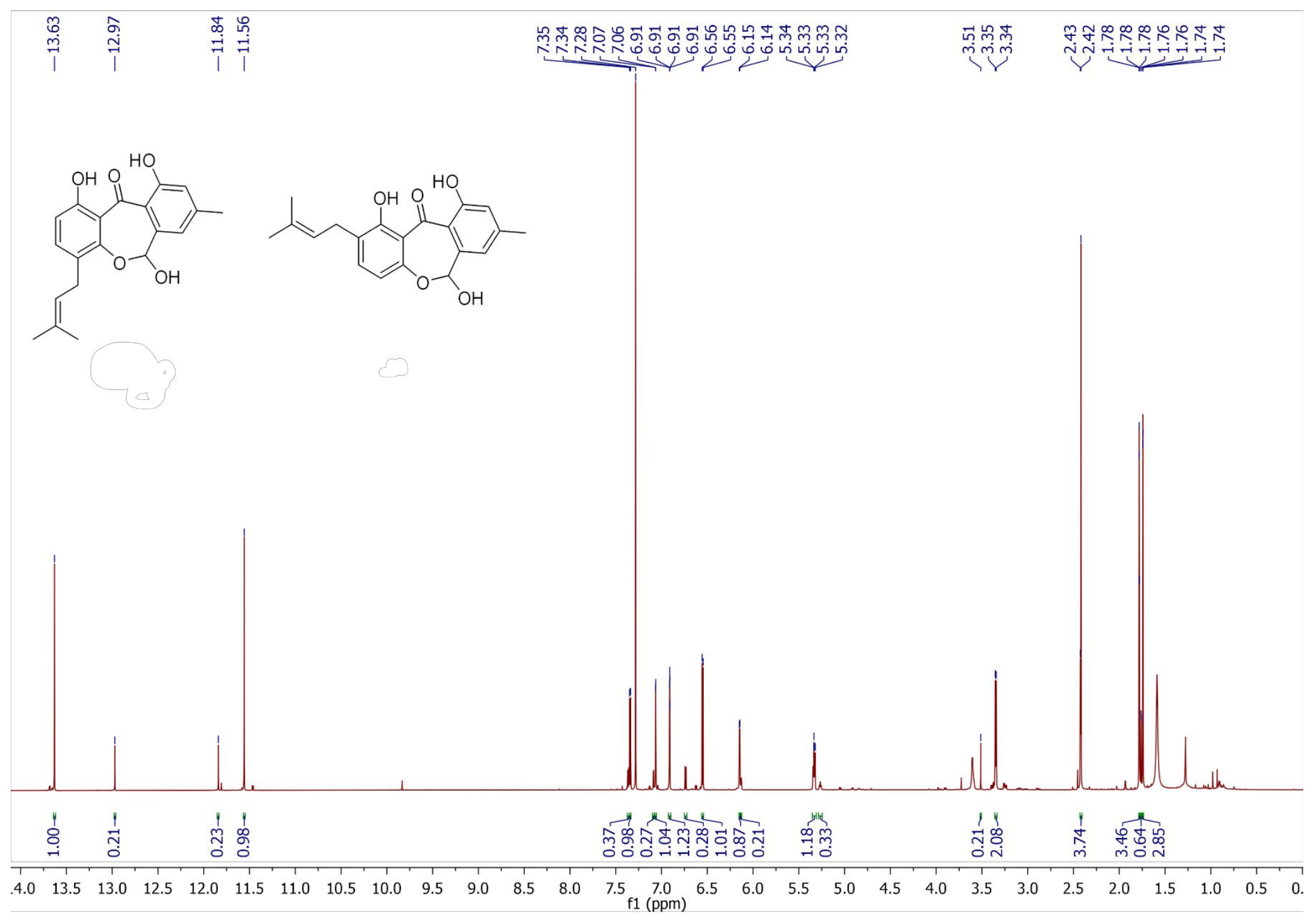

Figure S59. ${ }^{1} \mathrm{H}$ NMR spectrum of 11 and 12 in $\mathrm{CDCl}_{3}(700 \mathrm{MHz})$ 


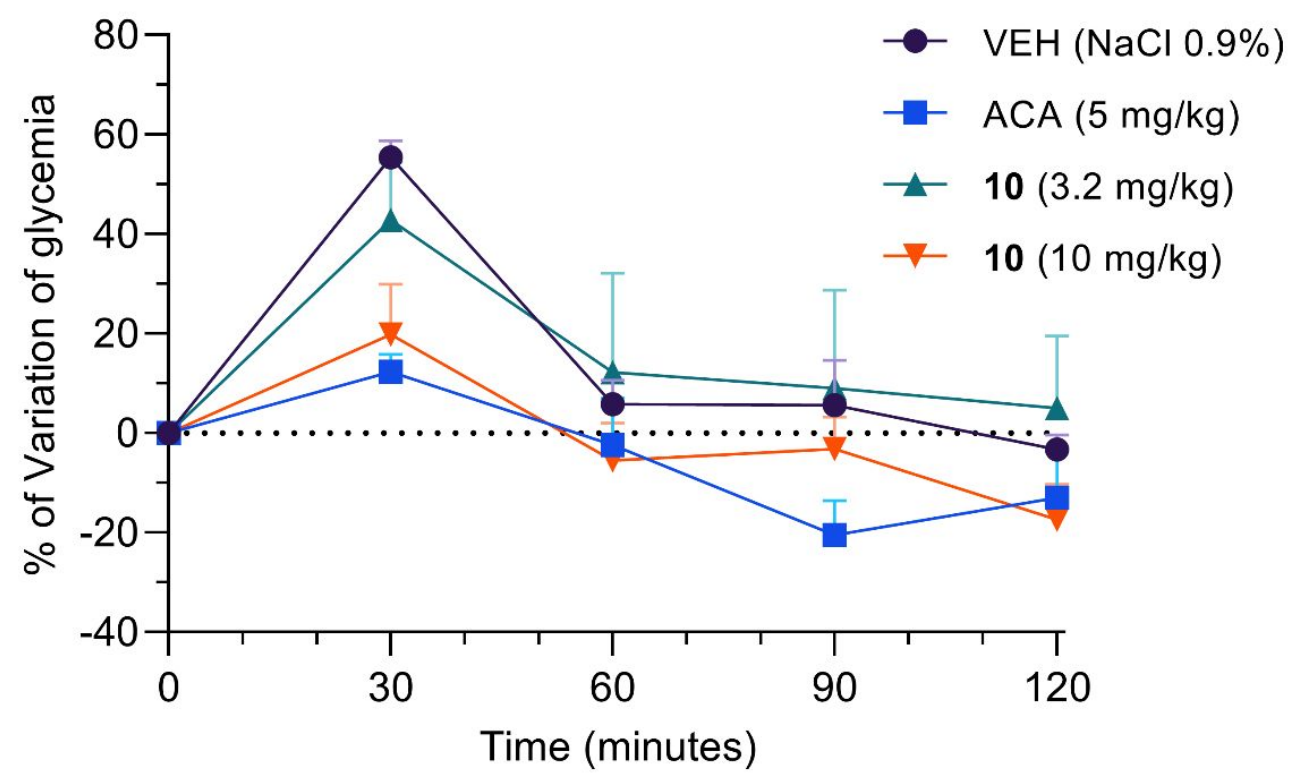

Figure S60. Sucrose tolerance test of malbrancheamide (10).
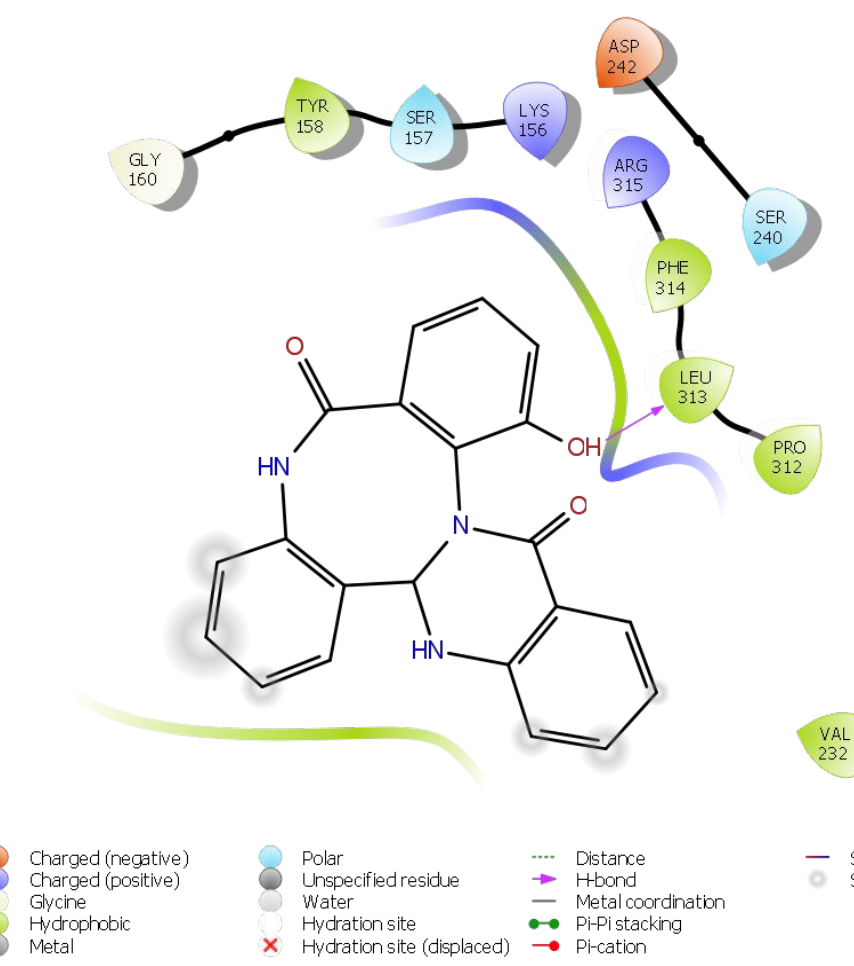

- Salt bridge
Solvent exposure

Figure S61. Docking analysis for $1(\alpha \mathrm{GHY})$. 

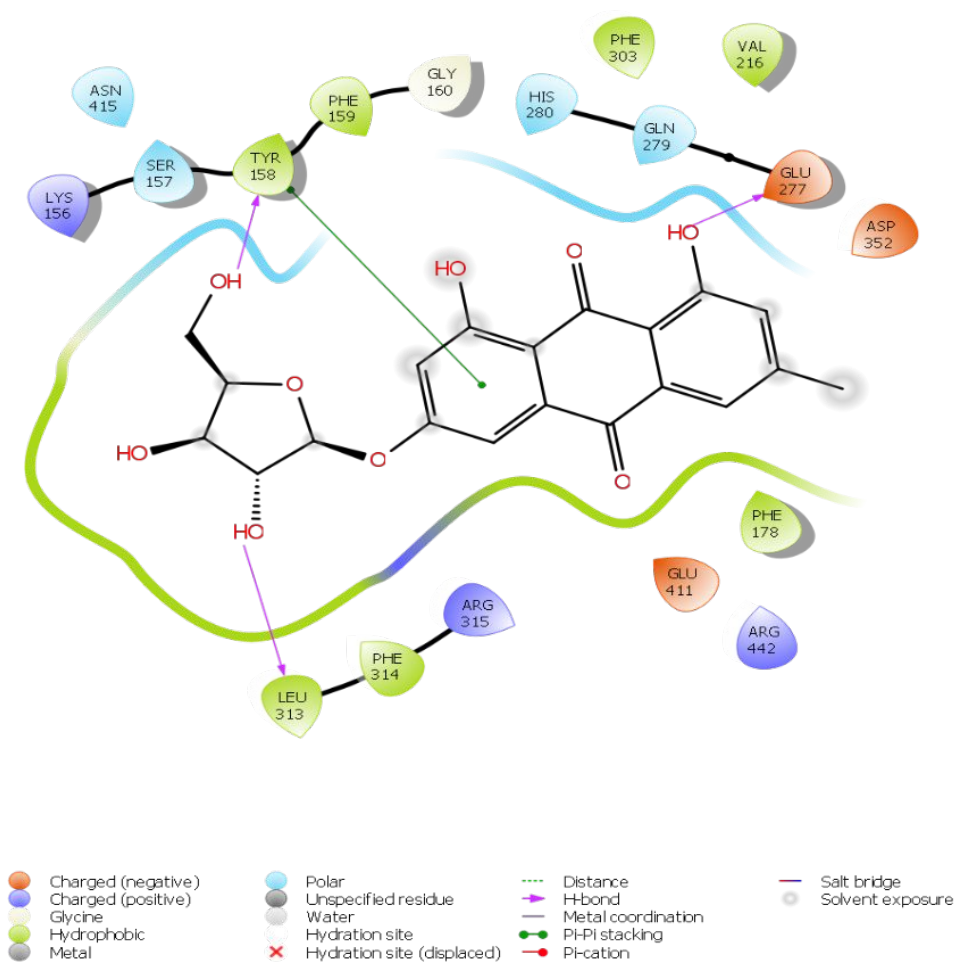

Figure S62. Docking analysis for 3 ( $\alpha \mathrm{GHY})$.

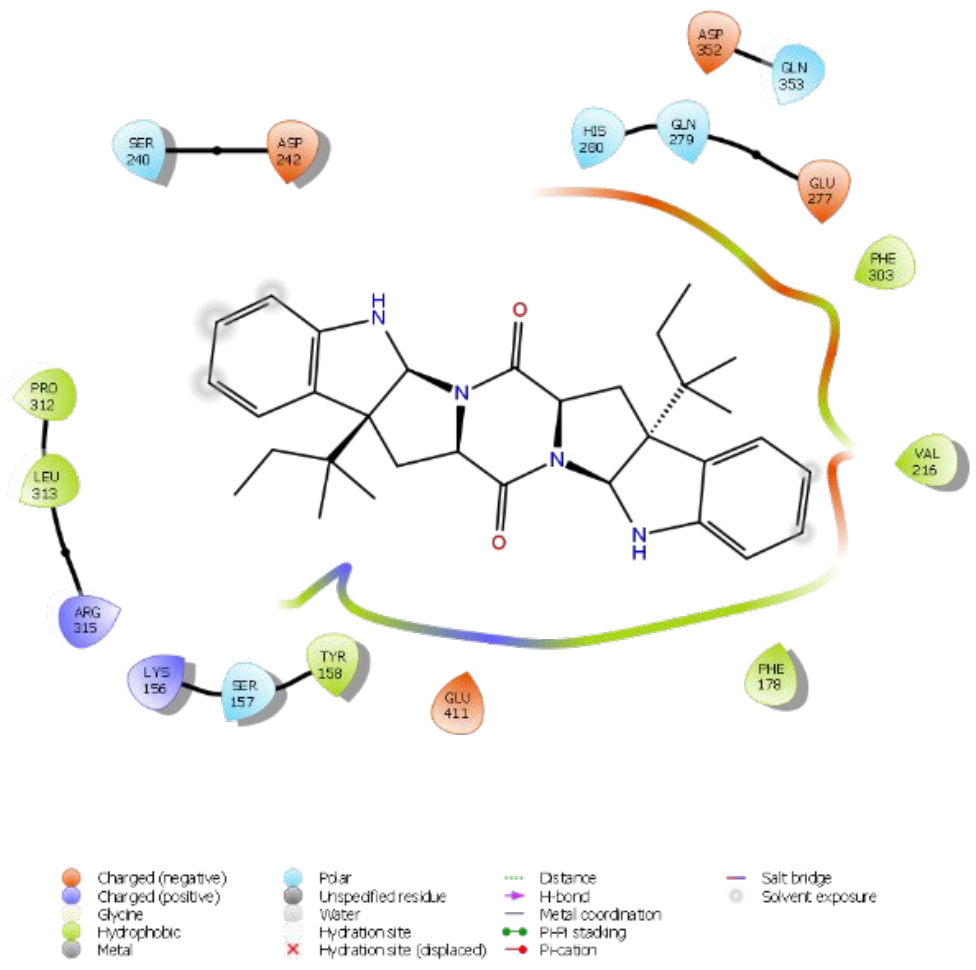

Figure S63. Docking analysis for $9(\alpha \mathrm{GHY})$. 


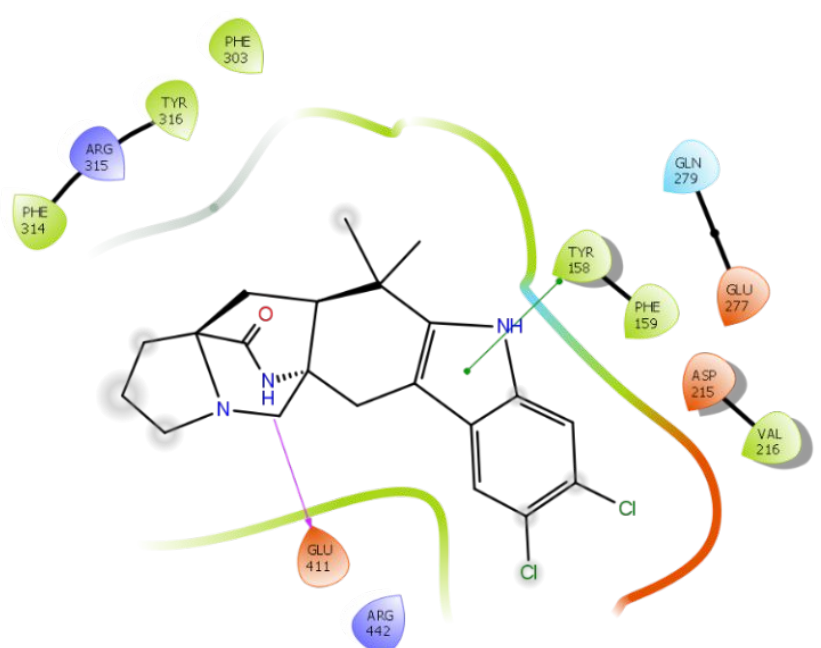

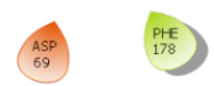

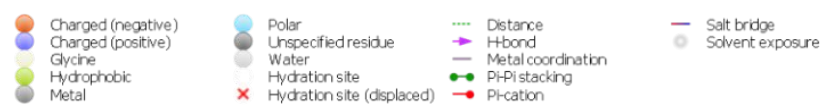

Figure S64. Docking analysis for $10(\alpha \mathrm{GHY})$.

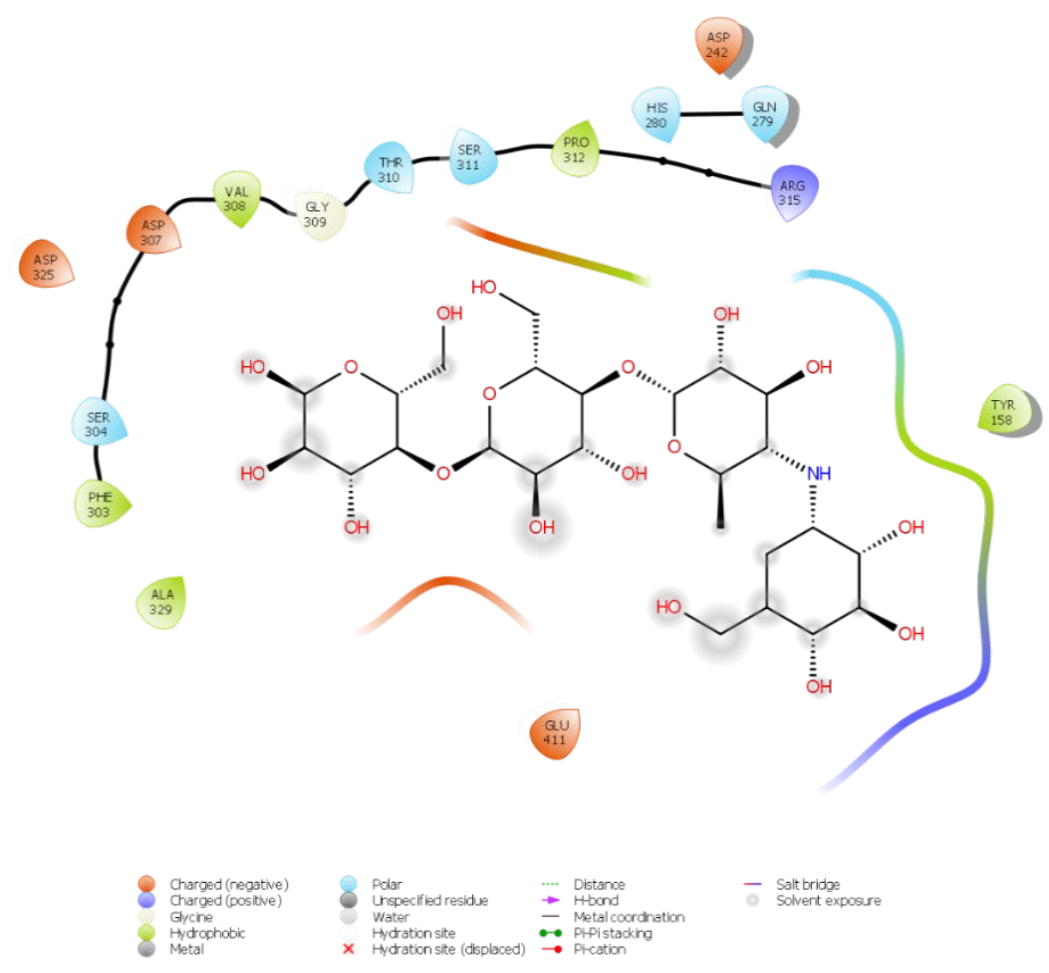

Figure S65. Docking analysis for acarbose ( $\alpha \mathrm{GHY})$. 

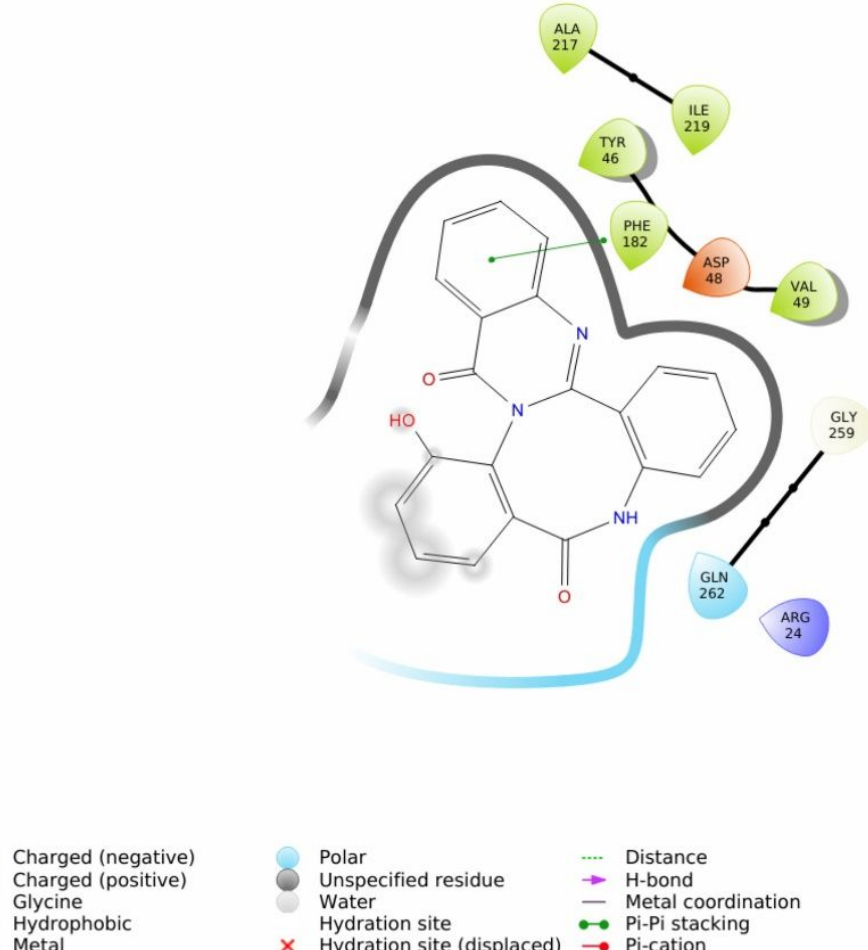

Figure S66. Docking analysis for 1 in (PTP-1B).

Charged (negative) Charged (positive) Glycine Hydrophobic Metal

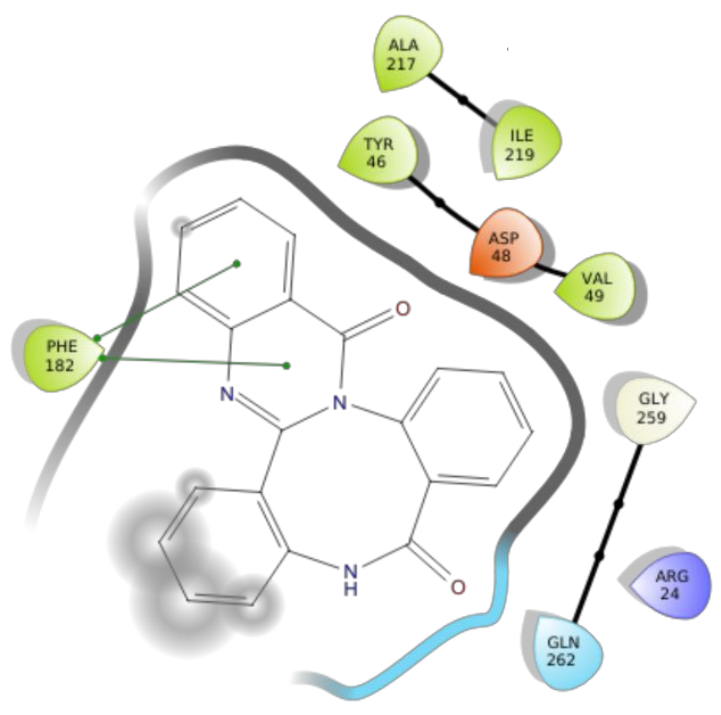

$\begin{cases}\text { Polar } & \cdots \text { Distance } \\ \text { Unspecified residue } & \rightarrow \text { H-bond } \\ \text { Water } & - \text { Metal coordination } \\ \text { Hydration site } & \longrightarrow \text { Pi-Pi stacking } \\ \text { Hydration site (displaced) } & \longrightarrow \text { Pi-cation }\end{cases}$

Figure S67. Docking analysis for 2 (PTP-1B). 
Charged (negative)

Charged (positive)

Glycine

Hydrophobic

Metal
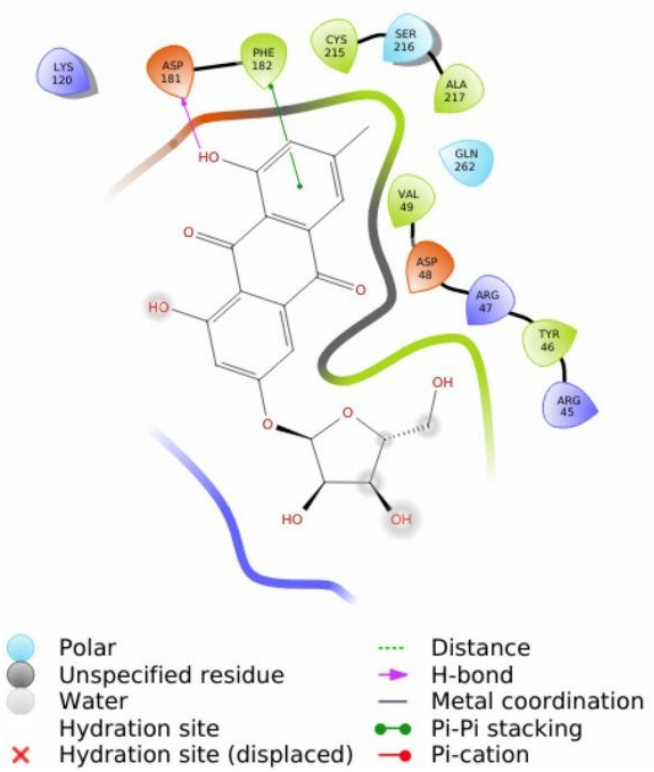

- Salt bridge Solvent exposure
- Metal coordination

$\rightarrow$ Pi-cation

Figure S68. Docking analysis for 3 (PTP-1B).

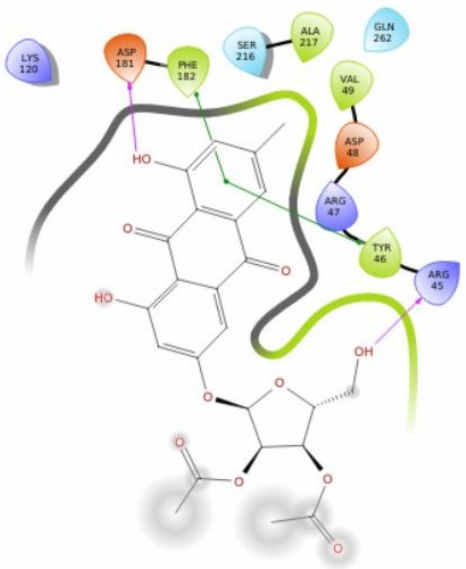

\begin{tabular}{|c|c|c|}
\hline $\begin{array}{l}\text { Polar } \\
\text { Unspecified residue }\end{array}$ & $\rightarrow$ & $\begin{array}{l}\text { Distance } \\
\mathrm{H} \text {-bond }\end{array}$ \\
\hline Water & - & Metal coordinatio \\
\hline Hydration site & $\bullet$ & Pi-Pi stacking \\
\hline Hydration site (displaced) & $\rightarrow$ & Pi-cation \\
\hline
\end{tabular}

Figure S69. Docking analysis for 4 (PTP-1B). 
Charged (negative) Charged (positive) Glycine

Hydrophobic

Metal

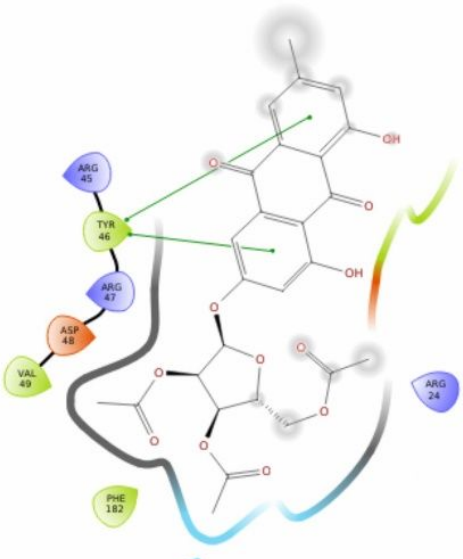

$\left(\frac{6,6}{262}\right)$

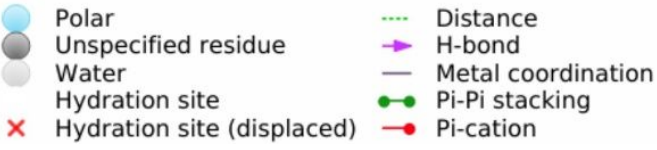

Polar $\quad$.... Distance

Unspecified residue

Water

$\rightarrow \mathrm{H}$-bond

- Metal coordination

Hydration site

$\because$ Pi-Pi stacking

$\times$ Hydration site (displaced) $\longrightarrow$ Pi-cation

Figure S70. Docking analysis for 5 (PTP-1B).

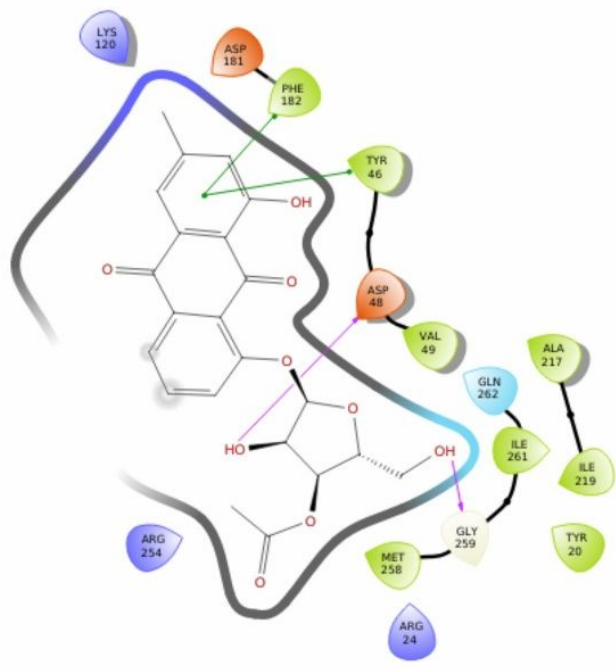

Charged (negative) Charged (positive)

Glycine

Hydrophobic

Metal

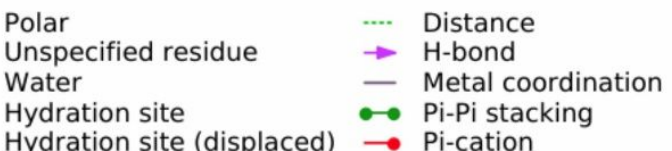

Hydration site (displaced) $\longrightarrow$ Pi-cation

Figure S71. Docking analysis for 6 (PTP-1B).
Salt bridge

Solvent exposure 


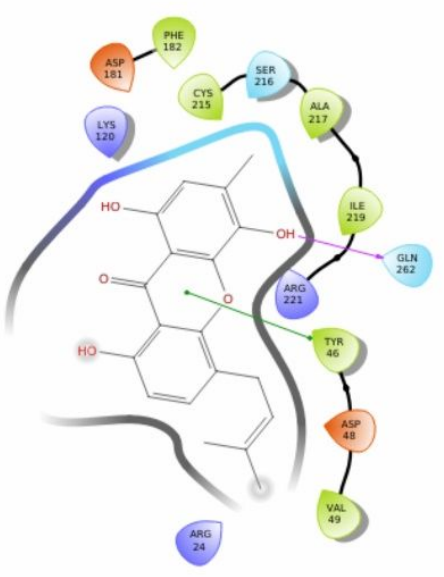

Charged (negative)

Charged (positive)

Glycine

Hydrophobic

Metal
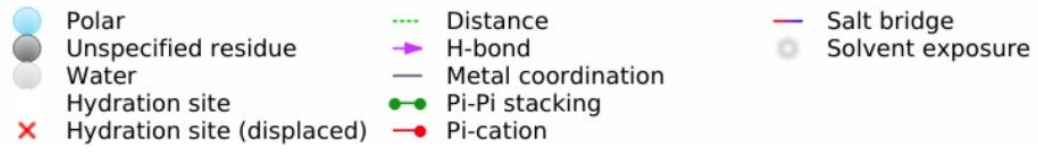

Figure S72. Docking analysis for 7 (PTP-1B).

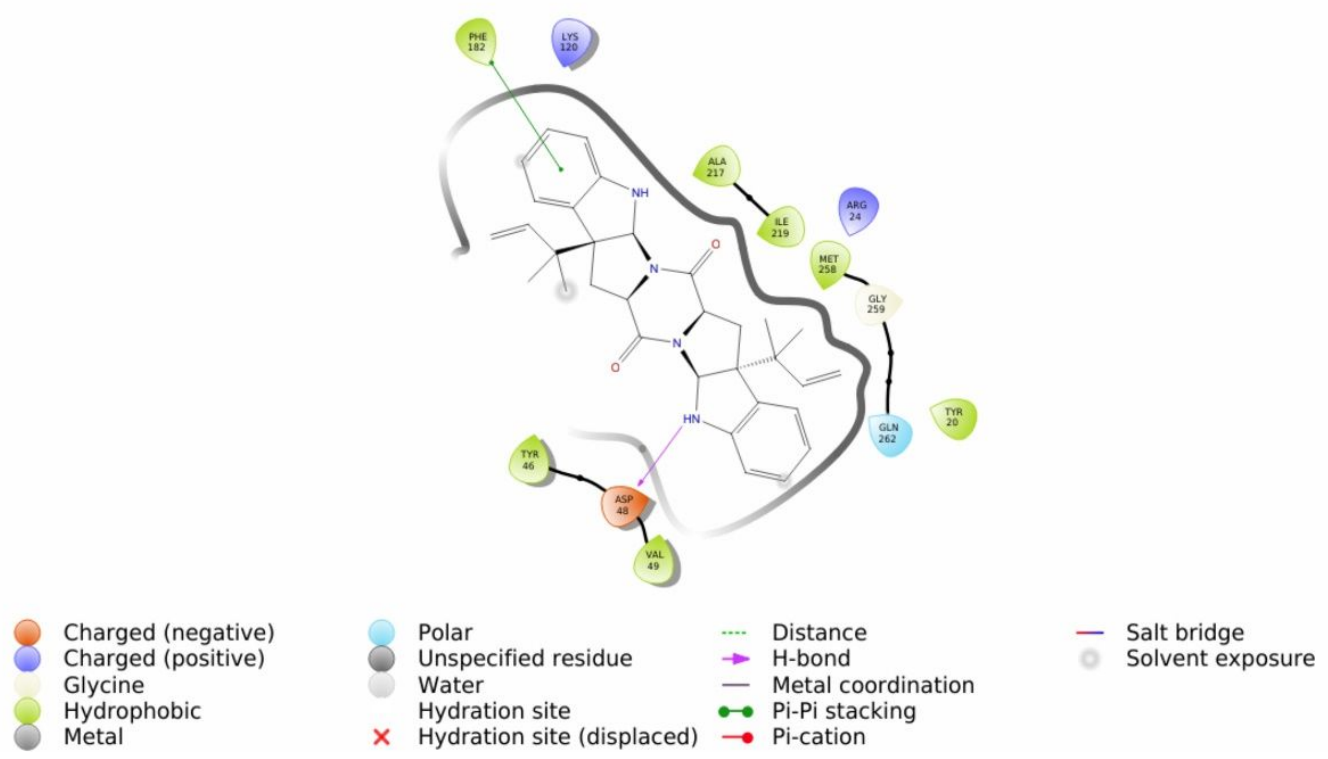

Figure S73. Docking analysis for 9 PTP-1B. 


\section{$\left(\begin{array}{l}\text { ASP } \\ 48\end{array}\right.$}

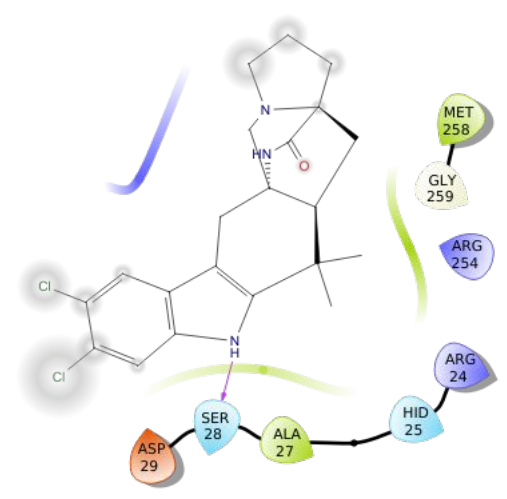

Charged (negative)

Charged (positive)

Glycine

Hydrophobic

Metal

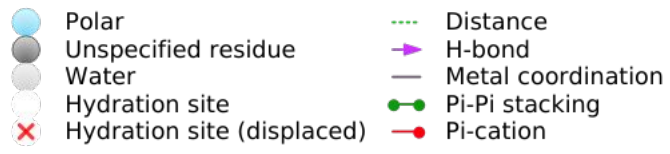

Figure S74. Docking analysis for 10 PTP-1B.

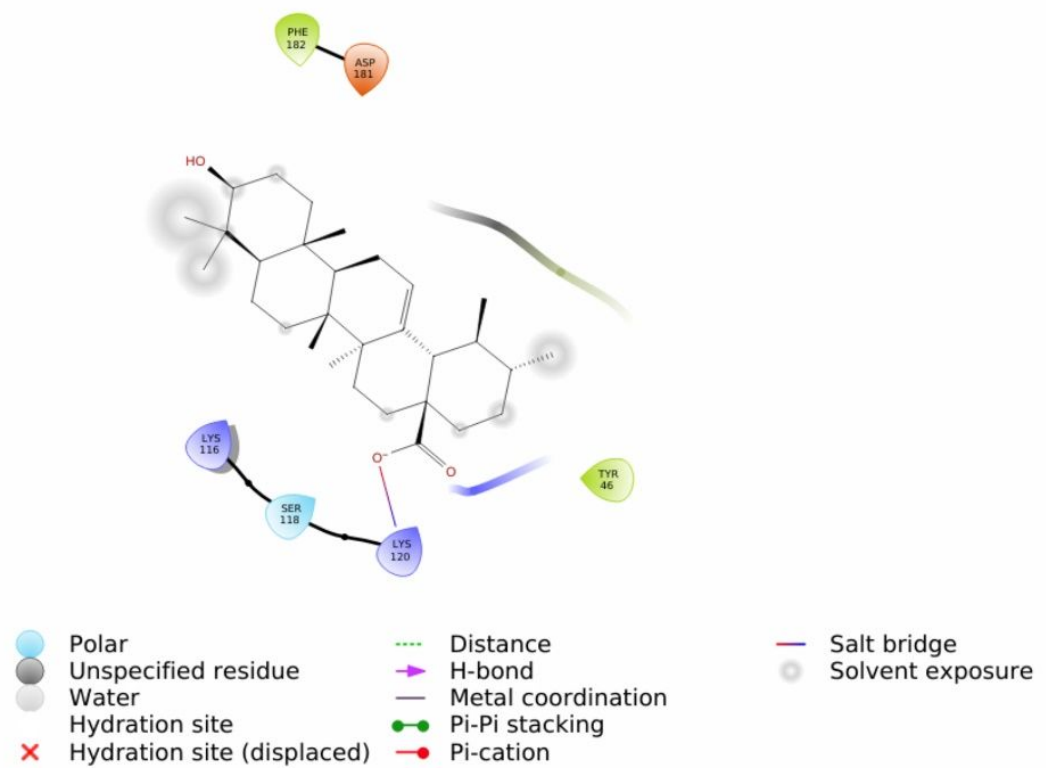

Figure S75. Docking analysis for Ursolic Acid.

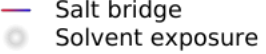
Unspeci
Hydration site $\quad \cdots$ Pi-Pi stacking

Charged (negative)

Charged (positive)

Glycine

Hydrophobic

Metal 


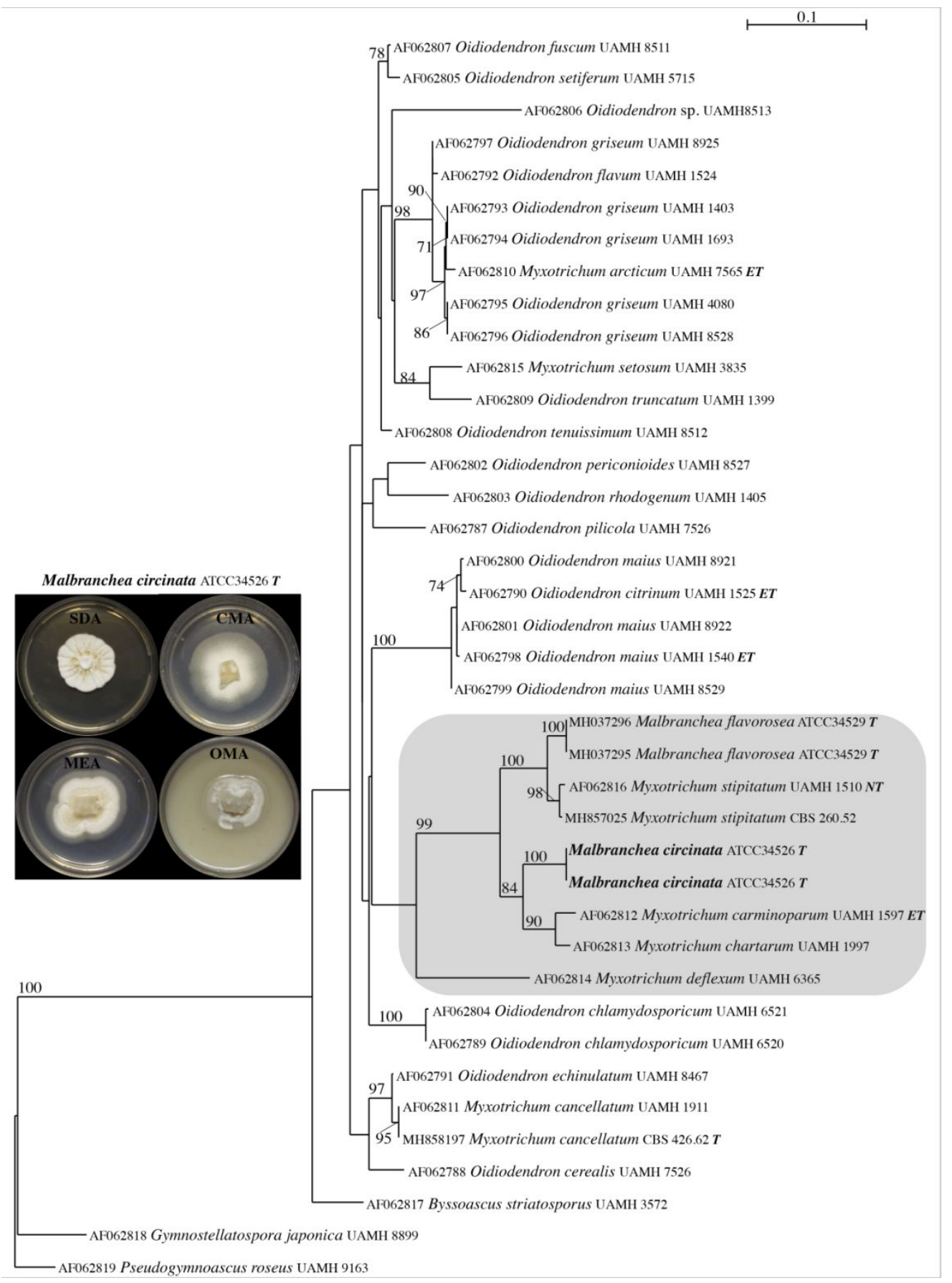

Figure S76. Phylogram of the most likely tree $(-\ln L=2909.24)$ from a RAxML analysis of 39 strains from the ITS region (519 bp). Numbers refer to RAxML bootstrap support values $\geq 70 \%$ based on 1000 replicates. Malbranchea circinata (ATCC: 34526) is phylogenetically related to Myxotrichum spp. (Leotiomycetes incertae sedis, Myxotrichaceae, Ascomycota). 14-Day old cultures from Sabouraud dextrose agar; Difco, Corn meal agar; Difco, Malt extract agar; Difco, and Oatmeal agar; Difco is shown. Symbols "T, ET, and NT" denote taxa reported as type, ex-type, or neo-type specimens. Bar indicates nucleotide substitutions per site. 


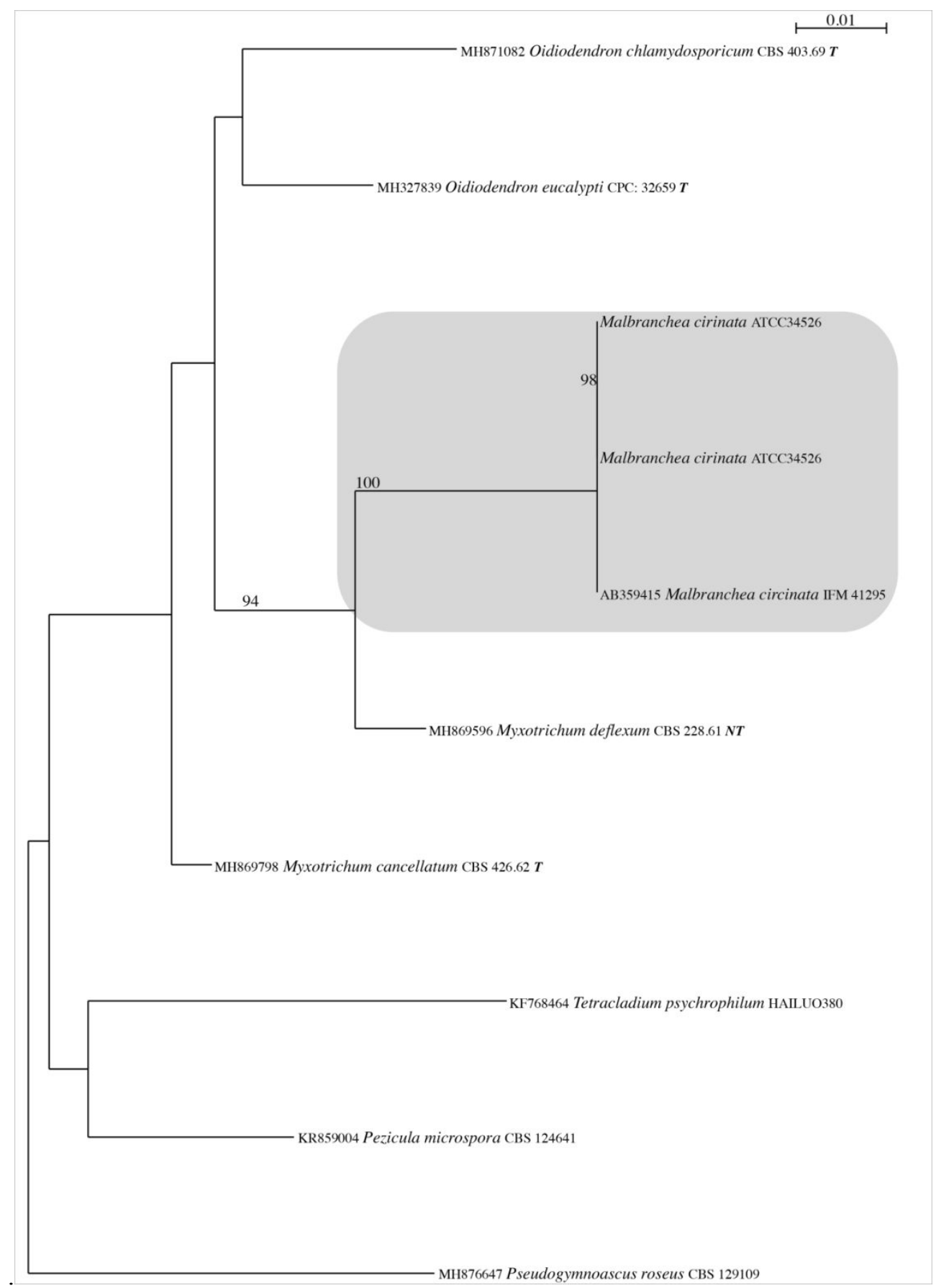

Figure S77. Phylogram of the most likely tree $(-\ln L=2091.2610)$ from a RAxML analysis of 10 strains from the partial 28SrDNA; LSU region (871 bp). Numbers refer to RAxML bootstrap support values $\geq 70 \%$ based on 1000 replicates. Malbranchea circinata (ATCC: 34526) is identical to M. circinata, IFM4126 (AB35914) and sister to Myxotrichum deflexum, CBS 228.61 (MH869596) (Leotiomycetes incertae sedis, Myxotrichaceae, Ascomycota). Bar indicates nucleotide substitutions per site. 


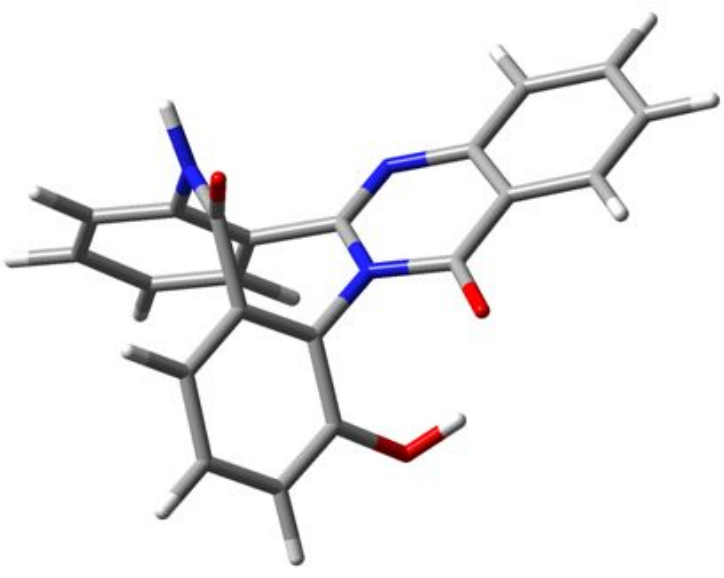

Conformer 1 (50\%)

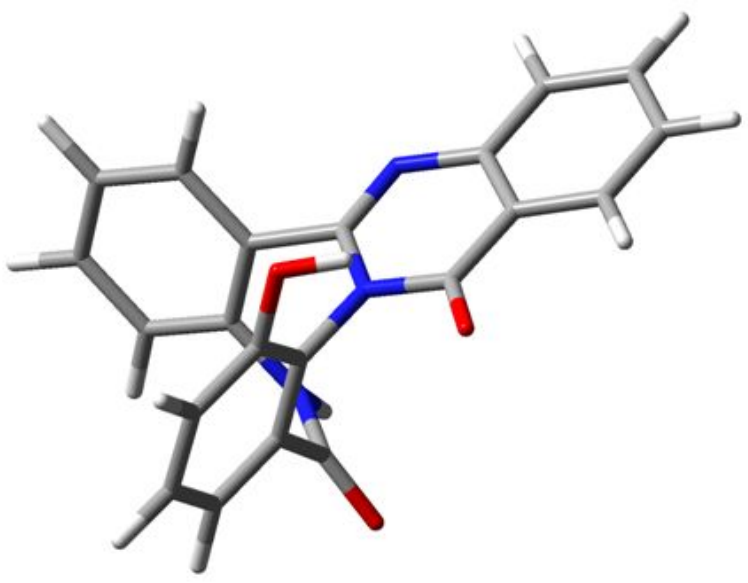

Conformer $2(50 \%)$

Figure S78. Conformations of compound 1 accounting for $100 \%$ of the conformational population. 
Table S1. Geometry Optimization and NMR Single-Point Calculations at DFT B3LYP/6-311+G(2d,p)//M06$2 \mathrm{X} / 6-31+\mathrm{g}(\mathrm{d}, \mathrm{p})$ Level of Theory for compound $\mathbf{1}$.

\begin{tabular}{|c|c|c|c|}
\hline Conformer & $\begin{array}{c}\text { Sum of electronic and } \\
\text { thermal Free Energies } \\
(\text { Atomic Units = Hartrees })\end{array}$ & $\begin{array}{c}\text { Energy }^{\mathrm{a}} \\
(\mathrm{kcal} / \mathrm{mol})\end{array}$ & $\begin{array}{c}\text { Molar Fraction } \\
(\mathrm{kcal} / \mathrm{mol})\end{array}$ \\
\hline 01 & -1197.399152 & -751379.4725277 & 0.5 \\
\hline 02 & -1197.399152 & -751379.4725277 & 0.5 \\
\hline
\end{tabular}

Table S2. Geometry Optimization and NMR Single-Point Calculations at DFT B3LYP/6-311+G(2d,p)//M06$2 \mathrm{X} / 6-31+\mathrm{g}(\mathrm{d}, \mathrm{p})$ Level of Theory for compound $\mathbf{2}$.

\begin{tabular}{|c|c|c|c|}
\hline Conformer & $\begin{array}{c}\text { Sum of electronic and } \\
\text { thermal Free Energies } \\
(\text { Atomic Units = Hartrees })\end{array}$ & $\begin{array}{c}\text { Energy }^{\mathrm{a}} \\
(\mathrm{kcal} / \mathrm{mol})\end{array}$ & $\begin{array}{c}\text { Molar Fraction } \\
(\mathrm{kcal} / \mathrm{mol})\end{array}$ \\
\hline 01 & -1122.196562 & -704189.1247539 & 0.5 \\
\hline 02 & -1122.196562 & -704189.1247539 & 0.5 \\
\hline
\end{tabular}


Table S3. Shielding tensors and NMR Single-Point Calculations at DFT B3LYP/6-311+G(2d,p)//M06-2X/6$31+g(d, p)$ Level of Theory for compound $\mathbf{1}$.

\begin{tabular}{|c|c|c|c|c|c|c|}
\hline \multicolumn{2}{|c|}{ Isotropic shielding constants $(\sigma)$} & \multicolumn{2}{|c|}{ Calculated chemical shift $(\delta)^{\mathrm{a}}$} & \multirow[b]{2}{*}{$\delta_{\text {calcd }^{\mathrm{c}}}$} & \multirow[b]{2}{*}{$\delta_{\exp }$} & \multirow[b]{2}{*}{$\left|\delta_{\text {exp }}-\delta_{\text {calcd }}\right|$} \\
\hline$\sigma_{01}$ & $\sigma_{02}$ & $\delta_{01}$ & $\delta_{02}$ & & & \\
\hline 23.62280 & 23.62280 & 7.73187 & 7.73187 & 7.73 & 7.80 & 0.068134 \\
\hline 48.79160 & 48.79150 & 125.87873 & 125.87883 & 125.88 & 126.83 & 0.951222 \\
\hline 49.83340 & 49.83340 & 124.88861 & 124.88861 & 124.89 & 126.57 & 1.681386 \\
\hline 40.38080 & 40.38070 & 133.87227 & 133.87236 & 133.87 & 134.99 & 1.117685 \\
\hline 28.04060 & 28.04070 & 145.60027 & 145.60017 & 145.60 & 147.28 & 1.679781 \\
\hline 57.34800 & 57.34790 & 117.74682 & 117.74691 & 117.75 & 120.50 & 2.753136 \\
\hline 48.84660 & 48.84680 & 125.82646 & 125.82627 & 125.83 & 127.57 & 1.743636 \\
\hline 23.47280 & 23.47280 & 7.87118 & 7.87118 & 7.87 & 7.93 & 0.05882 \\
\hline 23.78200 & 23.78200 & 7.58401 & 7.58401 & 7.58 & 7.63 & 0.045993 \\
\hline 22.99660 & 22.99660 & 8.31346 & 8.31346 & 8.31 & 8.31 & 0.003458 \\
\hline 14.08900 & 14.08900 & 158.85972 & 158.85972 & 158.86 & 160.98 & 2.120278 \\
\hline 20.82360 & 20.82380 & 152.45923 & 152.45904 & 152.46 & 154.4 & 1.940867 \\
\hline 40.02430 & 40.02450 & 134.21108 & 134.21089 & 134.21 & 136.35 & 2.139013 \\
\hline 45.60510 & 45.60520 & 128.90715 & 128.90705 & 128.91 & 131.16 & 2.252901 \\
\hline 39.21420 & 39.21420 & 134.98099 & 134.98099 & 134.98 & 133.09 & 1.890992 \\
\hline 48.10590 & 48.10600 & 126.53041 & 126.53032 & 126.53 & 128.55 & 2.019635 \\
\hline 48.78080 & 48.78090 & 125.88899 & 125.88890 & 125.89 & 127.65 & 1.761053 \\
\hline 50.59060 & 50.59060 & 124.16898 & 124.16898 & 124.17 & 126.13 & 1.961021 \\
\hline 23.97530 & 23.97530 & 7.40448 & 7.40448 & 7.40 & 7.63 & 0.225523 \\
\hline 24.12950 & 24.12950 & 7.26126 & 7.26126 & 7.26 & 7.4 & 0.138739 \\
\hline 24.25530 & 24.25530 & 7.14442 & 7.14442 & 7.14 & 7.18 & 0.035577 \\
\hline 24.02100 & 24.02100 & 7.36203 & 7.36203 & 7.36 & 7.47 & 0.107968 \\
\hline 51.28300 & 51.28430 & 123.51093 & 123.50969 & 123.51 & 121.75 & 1.760312 \\
\hline 45.05390 & 45.05390 & 129.43100 & 129.43100 & 129.43 & 130.95 & 1.518998 \\
\hline 40.91020 & 40.91000 & 133.36913 & 133.36932 & 133.37 & 134.22 & 0.850774 \\
\hline 23.65700 & 23.65700 & 149.76639 & 149.76639 & 149.77 & 152.65 & 2.883606 \\
\hline 55.17180 & 55.17270 & 119.81505 & 119.81420 & 119.81 & 117.78 & 2.034626 \\
\hline 56.15490 & 56.15550 & 118.88073 & 118.88016 & 118.88 & 116.91 & 1.970441 \\
\hline 24.35120 & 24.35120 & 7.05535 & 7.05535 & 7.06 & 6.92 & 0.135354 \\
\hline 24.43990 & 24.44000 & 6.97297 & 6.97288 & 6.97 & 6.9 & 0.072927 \\
\hline 24.09070 & 24.09070 & 7.29730 & 7.29730 & 7.30 & 7.29 & 0.007297 \\
\hline 4.69260 & 4.69250 & 167.78996 & 167.79006 & 167.79 & 170.79 & 2.999989 \\
\hline
\end{tabular}

$\begin{array}{cc}\text { MAE } & 1.28 \\ { }^{1} \mathrm{H}-\mathrm{MAE} & 0.08\end{array} \quad \mathrm{MAE}=\left|\Delta \delta_{\text {ave }}\right|=\frac{1}{N} \sum_{i=1}^{N}\left|\delta_{i}^{\text {comp }}-\delta_{i}^{\exp }\right|$

${ }^{a}$ Chemical shifts were derived from application of scaling factors (slope $=-1.0767$, intercept $=31.9477$ ) to the ${ }^{1} \mathrm{H}$ NMR shielding tensors and scaling factors (slope $=-1.0522$, intercept $=181.2412$ ) to the ${ }^{13} \mathrm{C}$ NMR shielding tensors computed at the B3LYP/6-311+G(2d,p)//M06-2X/6-31+G(d,p) level of theory. 
Table S4. Shielding tensors and NMR Single-Point Calculations at DFT B3LYP/6-311+G(2d,p)//M06-2X/6$31+g(d, p)$ Level of Theory for compound 2.

\begin{tabular}{|c|c|c|c|c|c|c|}
\hline \multicolumn{2}{|c|}{ Isotropic shielding constants $(\sigma)$} & \multicolumn{2}{|c|}{ Calculated chemical shift $(\delta)^{\mathrm{a}}$} & \multirow[b]{2}{*}{$\delta_{\text {calcd }^{\mathrm{c}}}$} & \multirow[b]{2}{*}{$\delta_{\exp }$} & \multirow[b]{2}{*}{$\mid \delta_{\text {exp }}-\delta_{\text {calcd }}$} \\
\hline$\sigma_{01}$ & $\sigma_{02}$ & $\delta_{01}$ & $\delta_{02}$ & & & \\
\hline 23.6296 & 23.6297 & 7.7256 & 7.7255 & 7.73 & 7.81 & 0.084496 \\
\hline 48.9712 & 48.971 & 125.7080 & 125.7082 & 125.71 & 126.93 & 1.221865 \\
\hline 50.2253 & 50.2251 & 124.5162 & 124.5163 & 124.52 & 126.55 & 2.033748 \\
\hline 41.0602 & 41.0601 & 133.2266 & 133.2267 & 133.23 & 135.19 & 1.96338 \\
\hline 27.8463 & 27.8464 & 145.7849 & 145.7848 & 145.78 & 147.17 & 1.385121 \\
\hline 56.1963 & 56.1965 & 118.8414 & 118.8412 & 118.84 & 120.61 & 1.768715 \\
\hline 49.4423 & 49.4423 & 125.2603 & 125.2603 & 125.26 & 127.68 & 2.419688 \\
\hline 23.5198 & 23.5198 & 7.8275 & 7.8275 & 7.83 & 7.95 & 0.122471 \\
\hline 23.8333 & 23.8333 & 7.5364 & 7.5364 & 7.54 & 7.67 & 0.133639 \\
\hline 23.1176 & 23.1176 & 8.2011 & 8.2011 & 8.20 & 8.32 & 0.118923 \\
\hline 12.6682 & 12.6681 & 160.2100 & 160.2101 & 160.21 & 161.61 & 1.399916 \\
\hline 20.5163 & 20.5163 & 152.7513 & 152.7513 & 152.75 & 153.7 & 0.948717 \\
\hline 39.8879 & 39.888 & 134.3407 & 134.3406 & 134.34 & 133.78 & 0.560667 \\
\hline 45.8131 & 45.8131 & 128.7095 & 128.7095 & 128.71 & 131.06 & 2.350534 \\
\hline 38.685 & 38.6849 & 135.4839 & 135.4840 & 135.48 & 136.31 & 0.826014 \\
\hline 47.5814 & 47.5812 & 127.0289 & 127.0291 & 127.03 & 128.94 & 1.911013 \\
\hline 48.9959 & 48.9959 & 125.6846 & 125.6846 & 125.68 & 127.73 & 2.045434 \\
\hline 50.223 & 50.2228 & 124.5183 & 124.5185 & 124.52 & 126.13 & 1.611562 \\
\hline 23.8866 & 23.8866 & 7.4869 & 7.4869 & 7.49 & 7.6 & 0.113142 \\
\hline 24.0954 & 24.0954 & 7.2929 & 7.2929 & 7.29 & 7.39 & 0.097068 \\
\hline 24.3 & 24.3 & 7.1029 & 7.1029 & 7.10 & 7.2 & 0.097093 \\
\hline 24.0265 & 24.0265 & 7.3569 & 7.3569 & 7.36 & 7.47 & 0.113076 \\
\hline 39.8252 & 39.8249 & 134.4003 & 134.4006 & 134.40 & 134.06 & 0.340447 \\
\hline 46.3587 & 46.3587 & 128.1909 & 128.1909 & 128.19 & 129.58 & 1.389067 \\
\hline 41.8562 & 41.8564 & 132.4701 & 132.4699 & 132.47 & 133.71 & 1.240032 \\
\hline 47.5072 & 47.5072 & 127.0994 & 127.0994 & 127.10 & 128.18 & 1.080589 \\
\hline 45.3813 & 45.3813 & 129.1198 & 129.1198 & 129.12 & 130.79 & 1.670156 \\
\hline 48.4202 & 48.4204 & 126.2317 & 126.2315 & 126.23 & 127.31 & 1.07839 \\
\hline 23.9845 & 23.9845 & 7.3959 & 7.3959 & 7.40 & 7.5 & 0.104068 \\
\hline 23.9135 & 23.9135 & 7.4619 & 7.4619 & 7.46 & 7.5 & 0.038126 \\
\hline 23.9907 & 23.9906 & 7.3902 & 7.3903 & 7.39 & 7.5 & 0.10978 \\
\hline 3.9896 & 3.9895 & 168.4581 & 168.4582 & 168.46 & 170.45 & 1.991865 \\
\hline 24.2389 & 24.2389 & 7.1597 & 7.1597 & 7.16 & 7.33 & 0.170346 \\
\hline
\end{tabular}

$$
\begin{array}{cc}
\text { MAE } & 0.99 \\
{ }^{1} \mathrm{H}-\mathrm{MAE} & 0.11 \\
{ }^{13} \mathrm{C}-\mathrm{MAE} & 1.49
\end{array} \quad \mathrm{MAE}=\left|\Delta \delta_{\text {ave }}\right|=\frac{1}{N} \sum_{i=1}^{N}\left|\delta_{i}^{\text {comp }}-\delta_{i}^{\exp }\right|
$$

${ }^{a}$ Chemical shifts were derived from application of scaling factors (slope $=-1.0767$, intercept $=31.9477$ ) to the ${ }^{1} \mathrm{H}$ NMR shielding tensors and scaling factors (slope $=-1.0522$, intercept $=181.2412$ ) to the ${ }^{13} \mathrm{C}$ NMR shielding tensors computed at the B3LYP/6-311+G(2d,p)//M06-2X/6-31+G(d,p) level of theory. 
Table S5. Inhibitory Effect of Compounds 1-12 Tested against Yeast $\alpha$-Glucosidase $(\alpha \mathrm{GHY})$ and Protein Tyrosine Phosphatase 1B (PTP-1B).

\begin{tabular}{ccc}
\hline \multirow{2}{*}{ Compound } & \multicolumn{2}{c}{ IC $_{\mathbf{5 0}}(\boldsymbol{\mu M})$} \\
\cline { 2 - 3 } $\mathbf{1}$ & $\alpha \mathrm{GHY}$ & $\mathrm{PTP}-1 \mathrm{~B}$. \\
$\mathbf{2}$ & $116.8 \pm 7.2$ & $17.3 \pm 0.9$ \\
$\mathbf{3}$ & $261.3 \pm 7.8$ & $106.2 \pm 1.6$ \\
$\mathbf{4}$ & $1428.8 \pm 47.3$ & $39.6 \pm 1.9$ \\
$\mathbf{5}$ & $>3 \mathrm{mM}$ & $27.9 \pm 0.7$ \\
$\mathbf{6}$ & $766.9 \pm 15.7$ & $25.5 \pm 2.6$ \\
$\mathbf{7}$ & $>3 \mathrm{mM}$ & $13.9 \pm 1.3$ \\
$\mathbf{8}$ & $288.1 \pm 16.5$ & $10.9 \pm 0.6$ \\
$\mathbf{9}$ & $57.4 \pm 0.2$ & $15.3 \pm 0.4$ \\
$\mathbf{1 0}$ & $71.3 \pm 0.7$ & $14.5 \pm 1.5$ \\
$\mathbf{1 1}$ and $\mathbf{1 2}$ & $>3 \mathrm{mM}$ & $30.3 \pm 1.2$ \\
Acarbose & $585.8 \pm 0.1$ & $27.8 \pm 0.1$ \\
\hline Ursolic acid & & \\
\hline
\end{tabular}

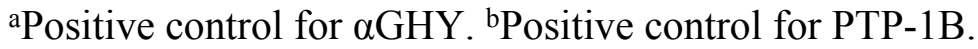




\section{Additional Experimental Details and Supporting References}

Molecular Identification of Fungal strain. For DNA extraction and PCRs were done in duplicate for each region. The sequence data were BLAST searched in NCBI GenBank to verify identity and sequence homology with other similar sequences in the public database. Based on a BLAST search of NCBIs GenBank nucleotide database, the closest hits using both ITS and LSU database were members of Oidiodendron Robak, and Myxotrichum Kunze in the Myxotrichaceae. To elucidate the phylogenetic placement of strain ATCC 34526, we downloaded the top matches of ITS and LSU sequences from GenBank and employed separate phylogenetic analyses. Maximum Likelihood phylogeny based on the ITS region showed that strain ATCC 34526, M. circinata was phylogenetically related to Myxotrichum spp. ${ }^{1}$ (Figure S76). Malbranchea circinata formed a strongly supported clade $(\geq$ 84\% bootstrap support) with Myx. carminoparum, and Myx. chartarum (Figure S76) along with $M$. flavorosea (ATCC 34529), and Myx stipitatum ( $\geq 99 \%$ bootstrap support). Our results therefore, suggest that the asexual M. circinata is phylogenetically related to the teleomorph genus Myxotrichum. ${ }^{1-3}$ Interestingly, Myx. carminoparum, and Myx. chartarum are stated in the literature to have to a Malbranchea asexual state. ${ }^{1}$ Maximum Likelihood analysis with partial LSU region also showed a similar result (Figure S77); $M$. circinata formed a strongly supported clade with a previously sequenced strain of M. circinata (IFM 41295; AB359415) ${ }^{4}$ and Myx. deflexum (CBS 228.61; MH869596).

Although, an ITS sequence of $M$. circinata (type; CBS 129.77) has been deposited in NCBI GenBank, our strain of $M$. circinata (ATCC 34526) displayed $\leq 40 \%$ sequence similarity with it. Instead another strain Malbranchea aurantiaca Sigler et Carmichael ATCC 34524 was 100\% similar to the ITS region of strain CBS 129.77 (unpublished data). Results from the sequencing and phylogenetic analyses performed herein suggest M. circinata (ATCC 34526) is affiliated to the Myxotrichaceae, Leotiomycetes incertae sedis; Ascomycota. Based on our preliminary sequencing analysis and the literature, it appears that the asexual genus Malbranchea is polyphyletic showing phylogenetic affiliations to both Onygenaceae (Eurotiomycetes) ${ }^{5-6}$ as well as Myxotrichaceae (Leotiomycetes, incertae sedis)2 ${ }^{2}$ (Figures S76 and S77).

\section{Supporting References}

(1) Hambleton, S.; Egger, K. N.; Currah, R. S. Mycologia 1998, 90, 854-868.

(2) Rebollar-Ramos, D.; Macías-Ruvalcaba, M. L.; Figueroa, M.; Raja, H. A.; González-Andrade, M.; Mata, R. J. Antibiot. (Tokyo). 2018, 71, 862-871. 
(3) L.; Carmichael, J. W. Mycotaxon 1976, 4, 349-488.

(4) Okada, K.; Takizawa, K.; Maebayashi, Y.; Nishimura, K.; Miyaji, M.; Fukushima, K. Mycoscience 1995, 36, 385-389.

(5) Sigler, L.; Hambleton, S.; Flis, A. L.; Paré, J. A. J. Stud. Mycol. 2002, 111-122.

(6) Sarrocco, S.; Diquattro, S.; Baroncelli, R.; Cimmino, A.; Evidente, A.; Vannacci, G.; Doveri, F. Mycol. Prog. 2015, 14, 112. 